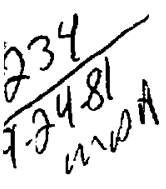

\title{
Calculations on Seismic Coupling of Underground Explosions in Salt
}

\author{
M. E. Heusinkreld
}

\section{January 20, 1981}
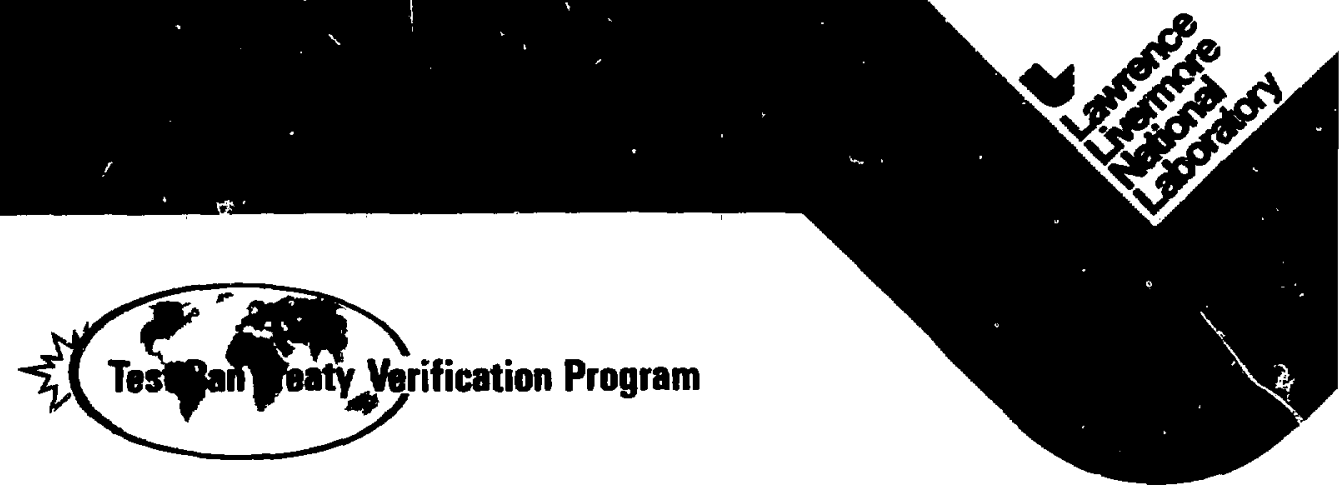


\title{
Calculations on Seismic Coupling of Underground Explosions in Salt
}

\author{
M. E. Heusinkveld
}

Manuscript date: January 20, 1981

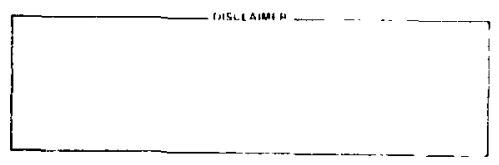

\section{LAWRENCE LIVERMORE LABORATORY} University of California - Livermore, California - 94550 
Abstract

Overview

Introduction

Purpose

Background

Salmon Experimental Resulis

Relation of jeismic Signals to Near-Region Measurements

Description of the $\operatorname{soc}$ Code . . * . * . . . . . . . . . 14

Determination of SOC Input Quantities . . . . . . . . . . . $1 \rightarrow$

Results of the SOC Calculations on Salmon. . . . . . . . . . Iy

Determination of the Elastic Ratius . . . . . . . . . . . . . 26

Discussion . . . . . . . . . . . . . . . . . . . . . . . . 32

Decoupling Casculations . . . . . . . . . . . . . . . . . . . . 33

General Description . . . . . . . . . . . . . . . . 31

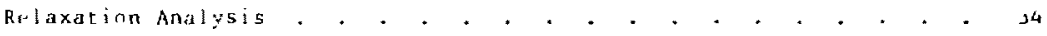

Relaxation with the $\operatorname{SoC}$ Code. . . . . . . . . . . . . . st

bynamic falcalations with the SoC Code--Step Input Preseures * . * 30

Comparison with other Results. . . . . . . . . . . . . . . . 45

Decoupling in Mined and in Explosively Formed Cavities. . . . . . . . . 4y

Qualitative Description . . . . . . . . . . . . . . . . 4y

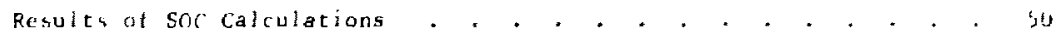

Decoupling Applications . . . . . . . . . . . . . . . . . 5j

Coupling of Nuclear and Chemical Explesions . * . . . . * . . s

Cowboy . . . . . . . . . . . . . . . . . . . . 54

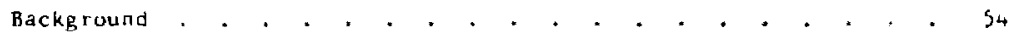

Tamped Cowboy Shots . . . . . . . . . . . . . . . 56

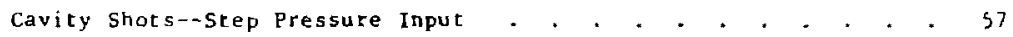

Calculation of Reverberating Pressure Sources . . . . . . . . . b3

Cowboy Shots--Reverberating Pressure lnput . + * + . + . . 63

Sterling. . . . . . . . . . . . . . . * . . . . . 72

Step Pressure Input . . . . * . . . . . . . * . . . 72

Reverberating Pressure Input . * . . . . . * . . . . . 73

Analysis of Cavity Reverberation . . . . . . . . . . . . . . 78 
Discussion

Acknowledgments

References

Appendix A: RDP and Displacement. Relations

Appendix B: Solution of Dynamic Equations with FFT . . . . . . . . 98

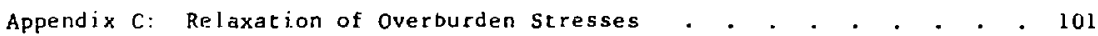

Appendix D: Scaling and Spectral Relations . . . . . . . . . . 106

Appendix E. RVP from the Sharpe Solution . . . . . . . . . - . 110

Appendix F: Resonant Frequency of the Gas in a Spherical Cavity . . 112 


\section{ABSTRACT}

In the Cuwboy and Dribble programs, a series of chemical and nuclear explosions were carried out in salt domes. Using data from these experiments, we have conducted a theoretical study of seismic coupling and decoupling of inderground explosions.

Computer calculations were made with the soc finite difference code to match the experimental data from the Salmon shor, which was part of the Dribble program. A fair match was obtained by using a shear strength of 8 MPa for the salt medium, although this value is considerably lower than the values obtained from cure samples. The resulting value for reduced displacement potential (RDP) for a tamped explosion was about $500 \mathrm{~m}^{3} / \mathrm{kt}$.

Further calculations were made on the variation of coupling with cavity radius using the input values for salt obtained from the salmon comparison, and using step application of cavity pressure. Comparative calculations were also made at twice overburden pressure and at higher values of strength. When the strength was doubied the residual RDP decreased more than when the overburden pressure was doubled.

We compared coupling in mined cavities with coupling in explosively formed cavities. Since the degree of weakening of the cavity walls in tie case of an explosively formed cavity was not known, no firm conclusion could be drawn on the comparative couplings. He also compared the calculated results obtained by sogers, Patterson, Latter et a:., Haskell, and Texhune et al. No significant differences were found.

Calculations were made on the coupled and decoupled shots in the Cowboy series and on the sterling event, with the material properties obtained in the salmon studies as computer input. It was found that application of step pressure into the cavities gave coupling values much lower than the experimental ones. When reverberating pressures at the cavity walls as calculated with SOC were applied, the agreement was better, although the computed couplings were still low. If it is assumed that the shear strength of the salt near the cavity is decreased by a factor of two because of the inelastic flow occurring near the cavity during the relaxation of the 
overburden stresses, then the agreement between the calculated and the experimental decoupling ratios is fairly good.

\section{OVERVIEW}

This report details the results of a theoretical study of seismic coupling and decoupling of underground explosions in a salt medium. A series of chemical and nuclear explosions was carried out years ago in salc domes for the Cowboy and the Dribble prograns to provide experimental data on seismic coupling for both tamped explosions and explosions in cavities. The Cowboy program consisted of a series of chemical explosions, and the Dribble program consisted of the camped nuclear Salmon event, the sterling nuclear event in the Salmon cavity, and an associated site calibration effort. This report presents the results of extensive computer calculations, which are in satisfaciury agreement with the experimental data. The calculations were extended to give general results on seismic coupling in salt.

The measure of seismic coupling for most of this work was the residual reduced displacerpent pocential (residual RDP). The decoupling associated with a shot in a cavity was expressed as the ratio of the resulting residual RDP to that of an equal-sized tamped shot.

Most of the investigations were made with the SoC computer program, which is a Lagrangian, finice difference, one-dimeisional code that siunulates stress wave propagation in earch media. Areas of investigation included the effect of the salt mechanical properties, the effect of initial cavity size, and the effect of the waveform of the pressure at the cavity wall.

The input parameters for SOC calculations were initially estimated from laboratory data on the salt, and were then adjusted to obtain agreement with the experimental results from the salmon event, a 5.3-kt nuclear explosion in a tamped geometry. These adjusted values for the salt medium were then used in calculations on Cowboy and sterling, as well as in general studies.

The adjustid set of properties of the medium included a failure shear strength of $8.0 \mathrm{MPa}$, zero porosity, a density ot $2.2 \mathrm{~g} / \mathrm{cc}$, a modulus oi rigidity of $13.9 \mathrm{GPa}$, Poisson's ratio of 0.25 , and a compressibility curve to give a sonic velocity of $4,357 \mathrm{~m} / \mathrm{s}$. The failure shear strength was the only parameter whose adjustment from the laboratory data was significant. In order to match observed displacements, the shear strength of 32.6 MPa measured 
in laboratory samples had to be reduced by a factor of four. Calculations showed that a dectease in shear strength increased the radius of inelastic motion, and increased the seismic coupling. Increasing the shear strength of the salt from 8.0 to $1.6 .0 \mathrm{MPa}$ decreased the inelastic radius and decreased the seismic coupling for all initial radii less than about $20 \mathrm{~m}$. Doubling the overburden pressure hac about the same effect on coupling as increasing the strength by apfroximately $50 \%$.

Further calculations were performed on the variation of coupling with cavity size. For these calculations, the values for the properties of the salt medium as determined in the Salmon calculations were used. The calculated residuat RUP increased from about $500 \mathrm{~m}^{3} / \mathrm{kt}$ for a point tamped source co $x^{\prime} 0 \mathrm{~m}^{3} / \mathrm{kt}$ tor an initial cavity radius of $2.0 \mathrm{~m} / \mathrm{kt}^{1 / 3}$, and decreased to appoximately $5.0 \mathrm{~m}^{3} / \mathrm{kt}$ for an initial cavity radius of 20) $n / k t^{1 / 3}$

fur the cilluistiuns on the cavities with the existing overburden pressure, it was recessary to obtain equilibrium stress configurations around tha caritios before the explosive energy was applied. This reiaxation was performed with tha STATIC aption in SOC. It was found that for both the Cowbay cavity shots and for Sterling, the salc near the cavities flowed inward in an inelastio nanner, which ingroduced the possibility that this salt was weakened oy the lnelastic flow.

In the calculatia.s on explosions in cavities, it was found that when the anergy if the explosion was homogeneously distributed throughout the cavity, the calculatei joak displasements were much less than the measured peak displacements in several of the cowboy shots. Calculations on the dynamics of the expissing within the unital cavity for each decoupled Cowboy shot showed that the actual pressure history at the cavity wall consisted of an initial spike, followed by reverberations in pressure. When this form of pressure wave was usef in the SOC inpuc, the calculated peak displacements more nearly matched the measured data for each cowboy event. In order to obtain good afreement between calculated and experimental decoupling ratios, we assumed that the shear strength of the salt adjacent to the cavities had bean decroased to 4 :Aa by the inelastic motion occurring in the earlier stress relaxation. With reverberating pressure and with a salt shear strength of $4 \mathrm{MPa}$ far the cavity calculations, the computed low frequency decoupling rat io for Cowbuy shot 12 (a detonation of $9291 \mathrm{~b}$ Pelletol in a spherical cavity with a b-ft radius) was 35 , as compared with a value of about 20 obtained from the 
seismic data. The calculated low frequency value for Cowboy shot 14, a detonation of $1902.4 \mathrm{lb}$ Pelletol in the same cavity, was 9, as compared with the experimental value of about 10 .

In the Sterling calculations, the duration of the pressure spike was quite short with respect to the characteristic period of the cavity, and the calculated peak displacements were not increased very much by the inelastic motions resulting from the spike. The calculated low frequency decoupling ratio with a shear strength of $4 \mathrm{MPa}$ was 65 , and that for an 8-MPa shear strengch was 108 . The experimental value was approximately 54.

INTRODUCTION

PURPOSE,

The purpose of this work was to analyze seismic decoupling of explosions in cavities in salt with the aid of ti?e soc finite difference code. We desired to nake computer calculations of the ground motions in the Salmon event, and by varying the constitutive relations of the salt medium, to match the salmon experimental data. Our aim was to then use these consticutive relations in SOC studies to determine the effects of cavity radius, overburden pressure, and pre-existing stresses in the medium on the coupling. We compared the SOC results with the results from theoretical analyses and with the experimental data from the Dribble and Cowboy projects.

\section{BACKGROUND}

The seismic coupling of underground explosions has been analyzed by Latter, Martinelli, and Teller. ${ }^{l}$ Their analysis showed that the distant ea: th displacement should vary as the first power of the energy release for the case where the pressure in the cavity is maintained for a long period of time, and as the $4 / 3$ power where the cavity pressure decays in a period proportional to the energy of the explosion, in a time range comparable with the periods of the seismic waves emitted. This mathematical analysis was used by Latter, LeLevier, Martinelli, and McMillan ${ }^{2}$ in calculating the amount of seismic decoupling that would be expected from an explosion in a large underground cavity. They compared the calculated seismic coupling from a 
cavity shot with the observed seismic coupling from the Ranier nuclear explosion in tuff, and concluded that a decoupling factor of $300 \mathrm{might}$ be possible in sa!t. In another paper, Latter, Martinelli, Mathews, and Mclililan ${ }^{3}$ considered the effect of pre-existing stresses around the cavity on the onset of inelastic motion resulting from an explosion in the cavity. They considered a medium that failed by plastic flow with a von Mises failure envelope, and concluded that these pre-existing stresses should have very little effect on the subsequent decoupling.

Haskel $l^{4}$ also analyzed this case, but assumed that the existing stress difference between the radial and the tangential stresses in all failed material would be the maximum permitted by the coulomb-Mohr failure criterion. He obtained quantitative resules for the expected decoupling as a function of cavity radius. Lewis and Treiman ${ }^{5}$ extended this analysis, and studied the requirement that the medium could sustain no tensile stress. Their analysis indicated that fajlure would occur at lower stress ievels than in the Haskell analysis, and that in sone applications three separate regions should result, the istasuosc including material that had failed in shear, the next region including material that had failed in tension, and the outermost being the elastic region.

Rodean has made further studies on decouplirg in cavities, ${ }^{6}$ and has analyzed the fraction of the total energy radiated in the seismic field. 7 He has compared the seismic signals from U.S. nuclear explosions in Nevada granite with the signals from French shots in Sahara granite.

An experimental program has been carried out to check these theoretical calculations. In the cowboy project, 8,9 chemical explosives were fired in tamped holes and in cavities in a salt medium near winnfield, Louisiana. Large decoupling factors for the shots in the cavities were observed.

Decoupling studies were extended to higher energies in the Dribble project, in which nuclear explosives were fired in the Tatum salt dome in Lamar County, Mississippi. In the salmon event ${ }^{10}$ a 5.3-kt nuclear device was exploded in a tamped hole in the salt. Eatensive ground motion measurements were made on this event. In the sterling event $11,12.30 .38-k t$ nuclear device was exploded in the salmon cavity, and again large decupling factors were measured.

Numerical calculations with finite disference computer programs have been carried through by Brode, ${ }^{13}$ by Rogers, ${ }^{14}$ by Patterson, ${ }^{15}$ by Godfrey et al. 16 and by Terhune, Snell, anci Rodean. 17 Early calculations by Brode 
indicated that strong reverberations should be expected in a nuclear explosion in a cavity. Later calculations by Patterson and by Godfrey gave similar results. Rogers made calculations of the Salmon stress-wave benavior, and included the effect of the overburden stress both in the horizoncal and in the vertical directions. Patterson made calculations on an explosion in the Salmon cavity, and first calculated a relaxed stress configuration around the cavity before the introduction of an explosior. He also made calculations on the effect of the high frequency spikes in pressure at the wall of the cavity to determine whether these spikes affected the distance within which the medium underwent inelastic deformation.

Terhune, Snell and Rodean made computer calculations on coupled and decoupled explosions in granite. They studied the peak in the coupling that appears at a cavity radius of about $2 \mathrm{~m}$ for a 1-kt explosion, and also calculated the exfected low frequency decoupling as a function of cavity radius.

SALMON EXPERIMENTAL RESULTS

In the Salmon event, Sandia Corporation made extensive masurements on the salt medium near the shot center, and these data formed the basis of comparison for normalizing the finite difference computer calculations for shots in salt as described in this report. The Sandia instrumentation included accelerometers and velocity meters emplaced in several holes located at distances out to $744 \mathrm{~m}$ from the shot. These holes were drilled into the salt medium to depths beneath the shot horizon. Ground motions were measured out to several seconds after shot time.

Figure 1 shows several particle-velocity vs time curves as measured by Perret ${ }^{18}$ of Sandia. Some of these velocity recoras have been obtained by integrating the accelerometer data. Perret et al. have integrated chese velocity records to give the displacement records of Fig. 2. Thess displacement curves show the initial transient displacement as the stress wave passed by, and then a long-time residual displacement. In Fin. 3 the residual displacements tabulated by Sandia are plotted, and represent the data that were to be matcied by computer calculations. 

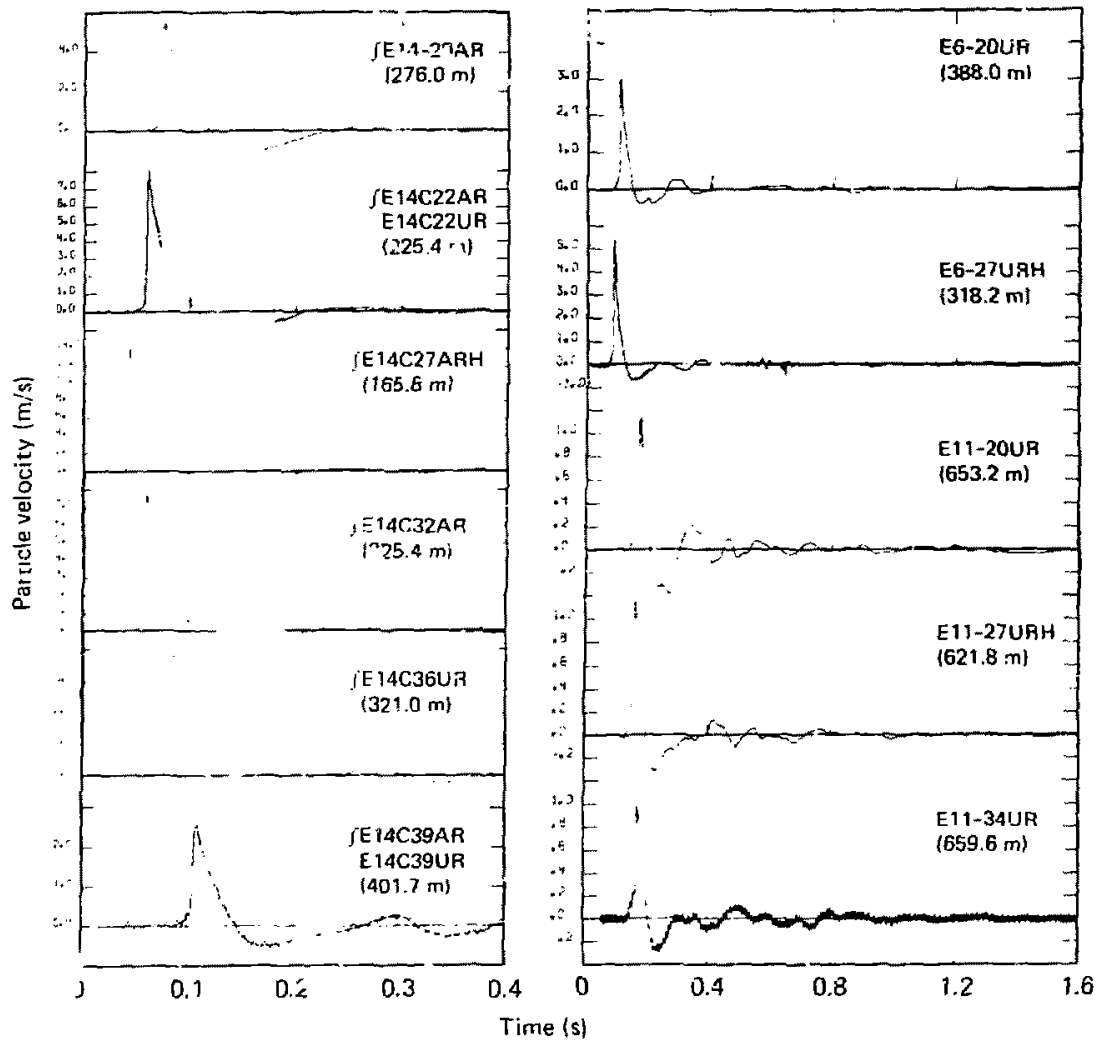

FIG. 1. Particle velr,it curves at severa: radial discances, as measured by San ia in the Salmon evenk. UR refers to velocity transducer data, and AR ref $2 r s$ to accelerometer data. R denotes radial direction and $h$ denotas ho:izontal direction. (Adapted from Reference 18.) 

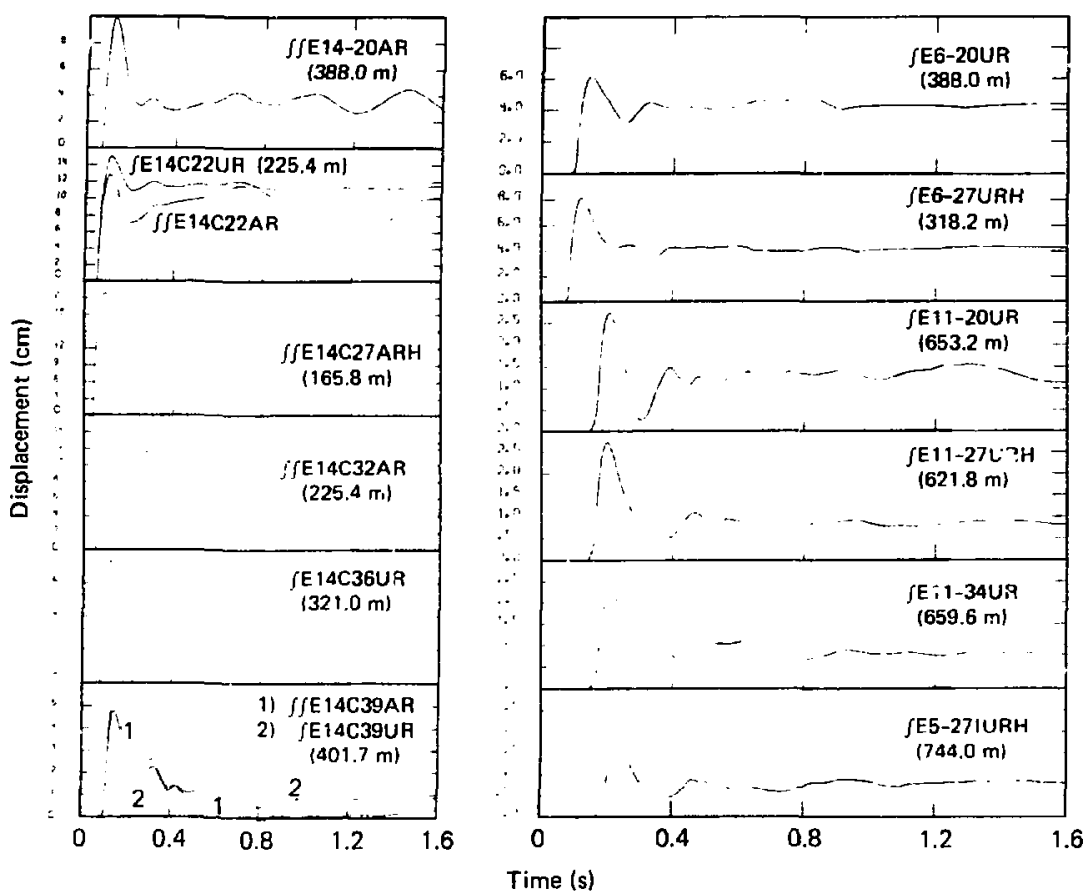

FIC. 2. Particle displacement curves at several radial distances, as measured by Sandia in the Salmon event. UR refers to velocity transducer data, and $A R$ refers to accelerometer data. R denotes radial direction and $H d a n o t e s$ horizontal direction. (Adapted from Reference i8.)

The reduced displacement potential (RDP) follows the relation

$$
z=-\frac{\partial}{\partial \tau}\left(\frac{\Downarrow(\tau)}{\tau}\right)
$$

where $z$ is the displacement and $\psi$ is the RDP. The quantiry $\tau$ is the retarded time, defined by the relation

$$
\tau=t-\frac{r}{c}
$$

where $c$ is the sonic velocity. Figure 4 shows the experimental RDP curves 


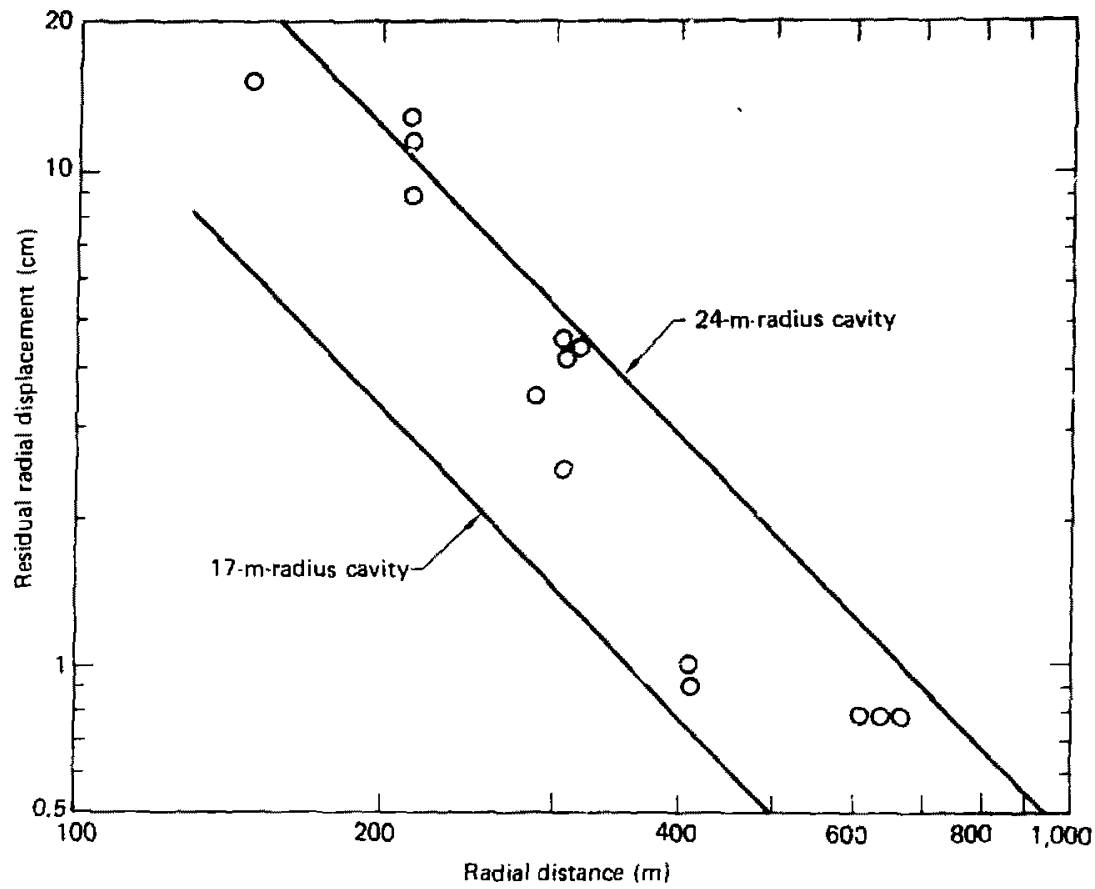

FIG, 3. Residual radial displacement us radial distance, from experimental data of Sandia in the Salmon event.

obrained by Sandia from the motion measurements un Salnon at several instrument locations.

Figure; shows the wave velocity curves ootained by Sandia. It contains che arrival times of the leading part of the transiert signal, representing the elastic wave; the arrival times of the beginning of the inelastic component; and the arrival times of the peak of the signal.

Perret has described the elastic wave as of very low amplitude, wich a transient pressure of only one-half to one MPa. The rate of radial atcenuation of this elastic waye was yery low, which indicates chat energy was being transferred continuously from the inelastic wave to the elastic wave as the composite wave moved oucward. Perrec placed the transition stress level between inelastic and elastic behavior at $1.95 \mathrm{MPa}$ radial stress. perret has 


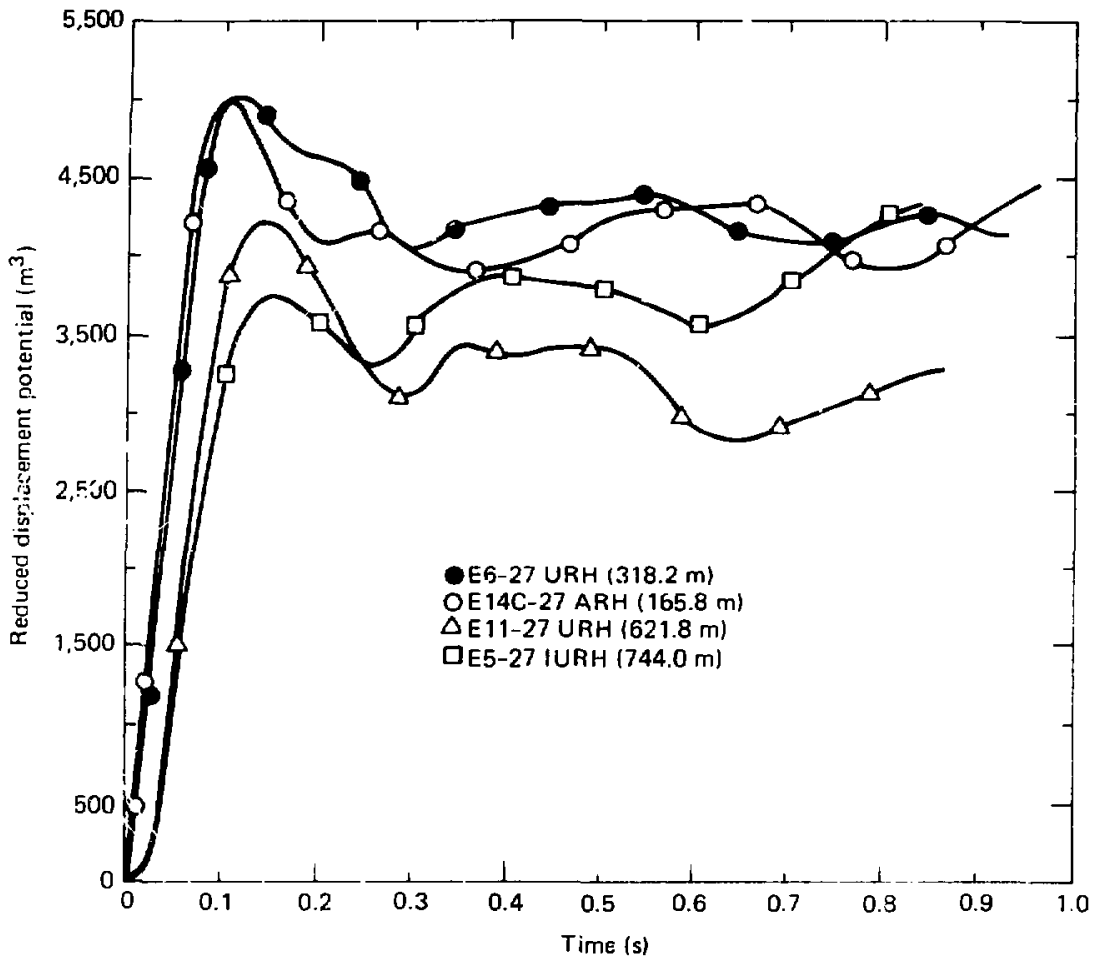

FIC. 4. Reduced displacemenc pocencial (RDP) vs time at several unstrument locations, as measured by Sandia in the Salmon event. IRR refers to velocity meter data and ARH refers to accelerometer data, all measured in the radial horizontal direction. (Adapted from Reference 18.)

not presented the reduced velocity potential (RVP) signals corresponding to the RDP curves of Fig. 4, so that the constancy of the RVP signal in the elastic rejion cannot be checked.

RELATION OF SEISMIC SIGNALS TO MEAR-REGION MEASUREMENTS

Measurements of earth mosion near an underground explosion, such as in the Salmon event, may span the range from highly inelastic motion very near the source to essentially elastic motion much further away. In addition, the 


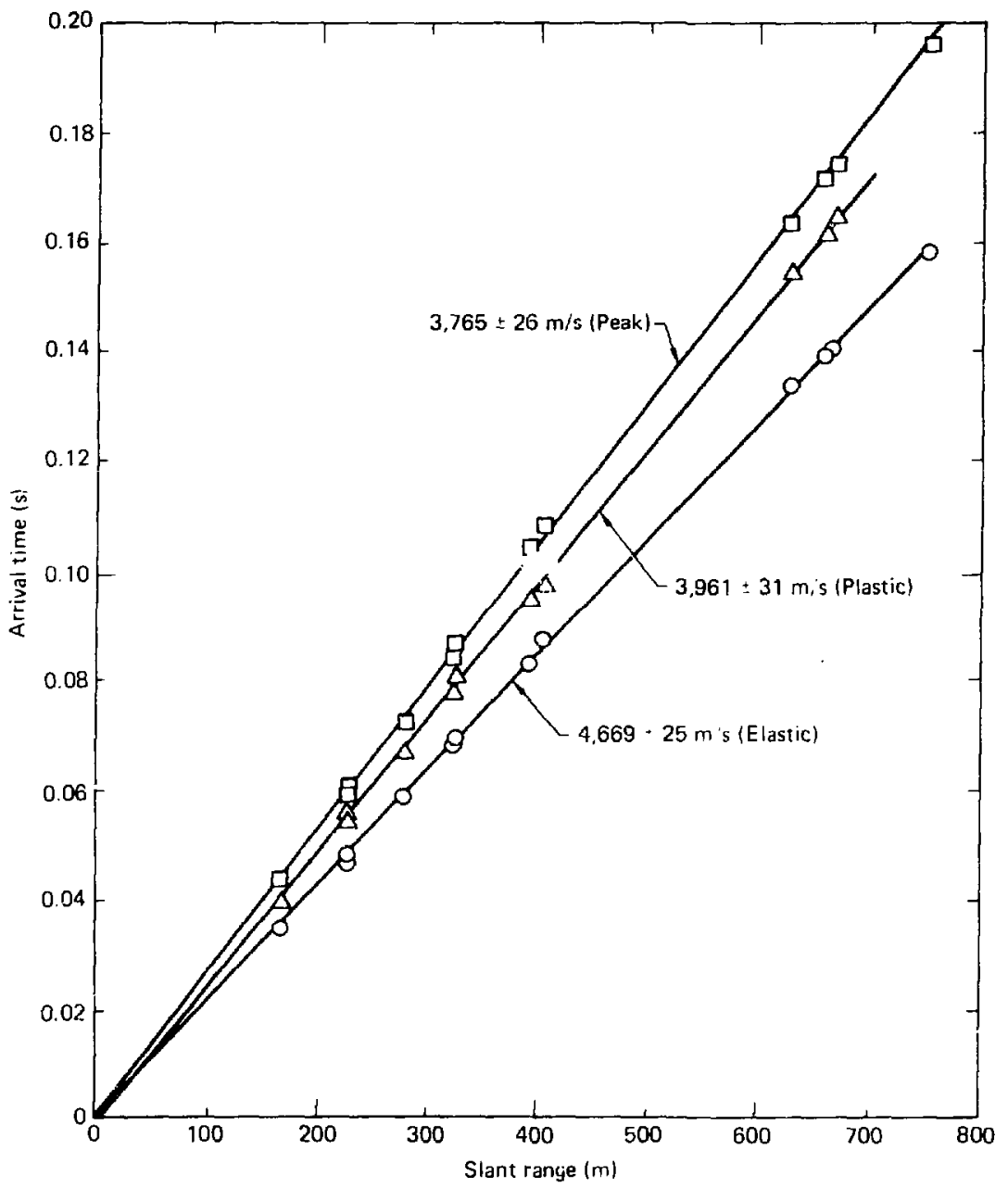

FIG. 5. Arrival time plots for several wave components, as measured by Sandia in the Salmon event. (Adapted from Reference 18.) 
disturbance contains both fields that radiate outward as waves and fields that do not follow the wave equation. Equation 1 may be expanded to the form

$$
z=\frac{\psi(\tau)}{r^{2}}+\frac{1}{c r} \frac{\partial \psi(\tau)}{\partial \tau}
$$

The first term on the right hand side can be defined as the induction field, varying as $l / r^{2}$, and the second term is the radiation field, varying as $1 / \mathrm{r}$. (The cerms near-field and far-field are avoided in these definitions because they have been criticized as being misleading and inaccurate.)

The induction field and radiation field complications can be avoided in the analysis of the experimental data measured near the explosion center by the use of RDP's and RVP's. These potential functions have wavelike properties in that tney move outward in spherical geometry without change in amplitude or shape when the medium remains in its elastic range. These functions are convenient measures of the seismic coupling, and the RDP's in particular have been used in determining decoupling ratios. As shown il Appendix $A$, the residua! $2 D P$ is a measure of the zero-frequency component of the distant seismic signals. It serves as a seismic coupling indicator up to frequencies approaching the characteristic frequencies of the cavity or of the excitation, but at higher frequencies the coupling has practically no dependence on the residual RDP. On the other hand, the RVP curve is directly related to the far-field displacements, and it is a better coupling indicator in many applications. Since the residual RDP was used as the measure of coupling in much of the earlier work, and because it gives the zero-frequency component of the RVP spectrum, the results of the $50 \mathrm{C}$ calculations are expressed botin in terms of residual RDP and peak RVP in this study.

In the frequency domain, the decoupling of an explosion in one geometry witn respect to another geometry is given by a decoupling-vs-frequency curve. The decoupling resulting from an explosion in a cavity as contrasted with a camped configuration is ordinarily defined as the ratio of the amplitudes of ine spectra of the displacements as observed at a distant point. This ratio is the same as the ratio of the spectra of the reduced velocity potentials, 1iscussed in appendix A.

In an incompressible medium, the residual radial displacement at some point in the medium is directly related to the change in cavity size. For a tampes asolosion, conservation of mass leads directly to the relation 


$$
z=\frac{a^{3}}{3 r^{2}}
$$

where a is final cavity radius.

Lines corresponding to cavity radii of $17 \mathrm{~m}$ and $24 \mathrm{~m}$ in an incompressible medium have been added to the displacement graph of Fig. 3. These lines bracket the experimental data fairly well, although this simple analysis neglects the possibilities of a decrease in salt density resulting from dilatation associated with shear failure of the salt, or of an increase in salt density because of pore collapse.

From Eq. (3) it is seen that when $\partial \psi / \partial \tau$ is zero, then the following simple relation holds:

$$
z_{r}=\frac{\psi(\infty)}{r^{2}}
$$

where ${ }_{r}$ is rasidual displacement and $\psi(\infty)$ is RDP at large $\tau$. This will occur after a stress wave has passed by and the medium has become quiescent again. Combining Eqs. (4) and (5), it is found that for a medium in which density does not change,

$$
\psi(\infty)=\frac{a^{3}}{3}
$$

For a tamped shot, where a is final cavity radius.

In the elastic regjme, the pressure must return to its original value after the stress wave has passed by, even though the cavity remains pressurized, so that the density of the surrounding medium must also return to its origincl value. If $a$ is the elastic radius and $z_{a}$ is the displacement at this radius, then Eq. (5) can be rewritten in the Eorm

$$
\psi(\infty)=a^{2} z_{a} .
$$

This equation is independent of the interactions occurring within the inelastic sphere, and will hold also at any larger radial distance. The RDP measurements on Salmon by Sandia shown in Fig. 4 do not indicate conclusively whether the region of constant RDP and elastic behavior was reached at the larger distances instrumented. 
The soc code has been described at length elsewhere, 19,20 so only a brief description is given here. It is a finite difference Lagrangian computer program, designed to solve problems in rock mechanics with impulsive loading. It is limited to problems in one-dimensional symmetry, such as radial motion in spherically symmetric geometry. Since it is a one-dimensional code, as contrasted with a 2-D code, fine zoning is possible without creating excessive computer storage or running time demands.

The pressure-volume and the failure relations between deviatoric stress and pressure can be put into SOC problems in tabular form. The failure mechanisms provided for are compaction, shear failure, and tensile failure. overburden pressure may be included either by use of an overburden option or by application of a pressure at the outer boundary of the grid.

It is possible in soc to specify a "static" option, in which an attenuation factor is applied to the velocities, so that the stresses throughout the grid gradially attain an equilibrium configuration. In this mode of operation, it is necessary that the loading and unloading paths be the same for the pressure-volume relation, and that the Lamé constants and the deviatoric stress yield curve be the same for unfailed and for failed material.

The SuC code contains an OVB option, by which the overburden pressure is not included in the compressibility data. The local overburden pressure becomes the "zero" pressure, and the compressions are measured from this point. When the pressure trops below overburden pressure, the compressibility curve is extrapolated back through this local ambient pressure linearly. This OVB option does not apply to the relation between failure shear stress and pressure. Tension can occur only when the maximum tensile principal stress in the medium completely cancels the corresponding component of compressive stress due to the overburden.

DETERMINATION OF SOC INPUT QUANTITIES

In the Salmon event the shot depth was $828.1 \mathrm{~m}$ below the ground surface, where the undisturbed overburden pressure was $18.1 \mathrm{MPa}$. This value of 
pressure was used at all radial distances and for most of the calculations on Salmon. The OVB option in SOC was used in all cases.

The medium at the Salmon shot point contained approximately $90 \% \mathrm{NaCl}$ and $10 \% \mathrm{CaSO}_{4}$ by weight, and had a density of about $2.2 \mathrm{~g} / \mathrm{cc}$. This medium was very dry, with a water of approximately $0.001 \%$ by weight. 21

The pre-shot velocity of dilatational waves in the Tatum salt was about $4,550 \mathrm{~m} / \mathrm{s}$ and the shear wave velocity was $2,539 \mathrm{~m} / \mathrm{s}$, as measured by Teledyne and by Birdwell. These velocity data lead to a value of poisson's ratio of approximately 0.25 . In the soc calculations this ratio was set at exactly 0.25 , partly to permit easier comparison with results from analytic formulas, which can be considerably simplified at this value because the two Lamé parameters are then equal to each other.

Perret's measurements of stress wave arrival times indicated an elastic wave velocity of $4,669 \mathrm{~m} / \mathrm{s}$, as shown in Fig. 5 . The reason for the disagreement with the pre-shot value of $4,550 \mathrm{~m} / \mathrm{s}$ is not known.

Triaxial compression tests have been performed on a number of cores from the vicinity of shot depth in the Tatum dome by the Bureas of Reclatration. 22 Under lateral confining stress of $20 \mathrm{MPa}$, the sait cores failed at an axial stress of $89.1 \mathrm{MPa}$ at $20 \%$ deformation. This gave a value of $32.6 \mathrm{MPa}$ for octahedral shear strength, defined by the relation

$$
\sigma_{0}=\frac{1}{3}\left[\left(\sigma_{1}-\sigma_{2}\right)^{2}+\left(\sigma_{2}-\sigma_{3}\right)^{2}+\left(\sigma_{3}-\sigma_{1}\right)^{2}\right] 1 / 2
$$

where $a_{1}, a_{2}$, and $a_{3}$ are principal stresses.

Heard, Abey, Bonner, and Duba ${ }^{23}$ have made extensive measurements on the properties of polycrystalline NaCl, including compressibility and shear strength. They tested samples of pure fine-grained salt that had been compacted to about one percent porosity. Among other results, they found that shear failure stress remains quite constant at about $40 \mathrm{MPa}$ over a wide range of confining pressure, as shown in Fig. 6. They observed a strain hardening effect, in that larger values of shear stress are required for larger values of inelastic deformation. They also observed that the brittle-ductile transition occurred at a pressure of about $3 \mathrm{MPa}$, and that failure in the brittle regime is preceded by volumetric expansion.

The sainfles tested by Heard et al, were composed of pure polycrystalline $\mathrm{NaCl}$, while the Salmon medium contained $10 \% \mathrm{CaSO}_{4}$, so that considerable 


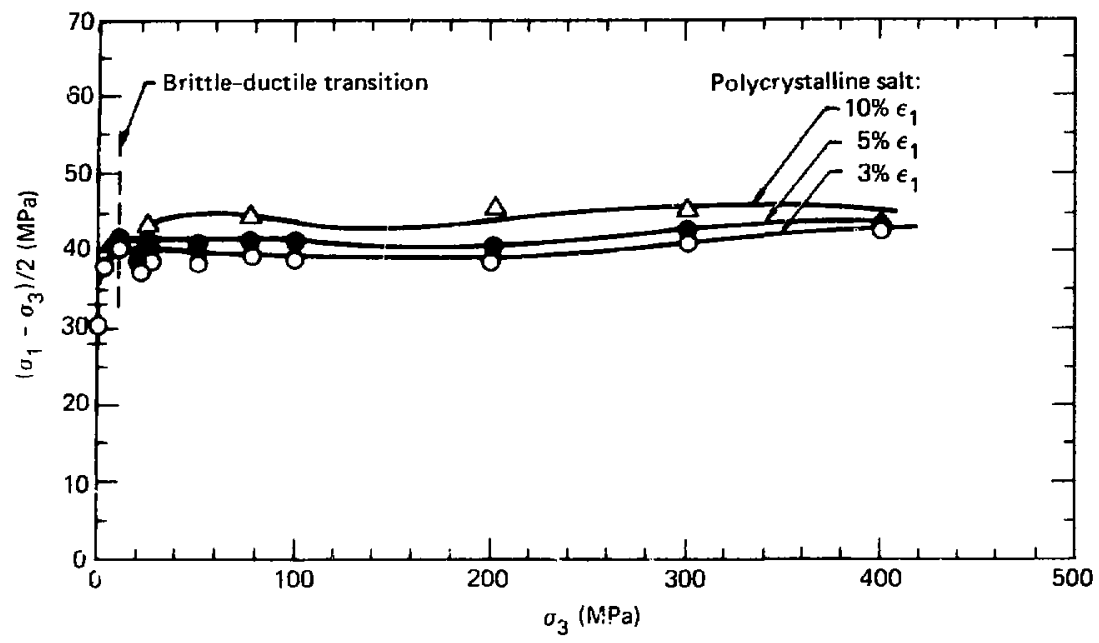

FIC. 6. Measurements of shear streagth vs confining pressure on polycrystalline Nacl at permanent strains of $3 \%, 5 \%$, and $10 \%$. (Adapted from Reference 23.)

differences might be expected in the failure levels, although the Salmon salt might still be expected to exhibit plastic failure.

Since the samples tested by Heard et al. contained $1 \%$ air-filled porosity, their measurements showed much $n$ re compressibility than the Salmon salt. Preliminary SOC calculations were made using as input the colstitutive relations given by Heard, et al, but the calculated residual displacements at a distance of some hundreds of meters from the explosion center were toward the center rather than away from it. In the version of the SOC code used for these calculations, the pores were collapsed by the outgoing stress wave, and when the stress wave had passed by, the medium rebounded to a position nearer the center than the original position. There is no provision in this version of SOC for either reopening the pores when the negative phase of the wave arrives, or of providing for dilatancy upon brittle shear failure. Although a version of SOC exists that has been designed to overcome these difficulties, it is very slow-running. ${ }^{24}$ The gas-filled porosity was set at zero to overcome the difficulty with $S O C$ and to match the actual Salmon salt porosity more closely. 
The PMUMAT code, developed by Butkovich 25 and modified by Sne11, 26 was used to generate a more realistic compressibilicy curve for the Tatum salt, with zero gas-filled porosity. The composition was set at $91 \%$ Nacl and 9\% limestone, since the $\mathrm{CaSO}_{4}$ component was not available in the PMUMAT library. Although the salt was known to be dry, $0.4 \%$ by weight of water was added in the input to PMUMAT to make the medium more compressible and to lower the sonic and plastic wave velocities. This procedure did give the additional compressibility that was required for the resulting wave velocities to match the field data. Figure 7 shows the resulting compressibility curve. Although the PMUMAT code aiso provides shear strength data, this option was not used because the resulting values of shear strength were much hightr than the known values for salt.

The results of the PMUMAT calculation gave $23.2 \mathrm{GPa}$ as a value for bulk modulus $K \rightarrow t$ low stress levels. At a Poisson's ratio of 0.25 and a density of 2.2 , the resulting elastic wave velocicy is $4,357 \mathrm{~m} / \mathrm{s}$, which is appreciably slower than the experimentally determined velocity of the low amplitude sonic waves of Salmon salt.

A grid extending out $1,600 \mathrm{~m}$ was used in these Salmon calculations. This grid was divided into 692 cells by the soc automatic zoner. With this grid size, the stress waves could be followed for about $0.4 \mathrm{~s}$ before reflections from the outer boundary would return and would begin to obscure the outgoing wave form.

The fine details of the geometry of the energy source and the time history of the energy emission were not considered in the SOC calculations of salinon because the very high frequency behavior was not of interest, and becaus: most of the zones in the salt were too large to permit accurate calculation of the effects of these fine source details. Instead, the source was represented by a sphere of iron vapor $50 \mathrm{~cm}$ in radius and of a density to give a total source mass of $1,152 \mathrm{~kg}$. The $5.3 \mathrm{kt}$ of energy was applied uniformly over this volume of vapor.

Table 1 gives the values used for the various soc input quantities. Also shown are corresponding values used by Rogers, ${ }^{14}$ patterson, ${ }^{15}$ and Terhune et al. 17 in their finite difference calculations. 


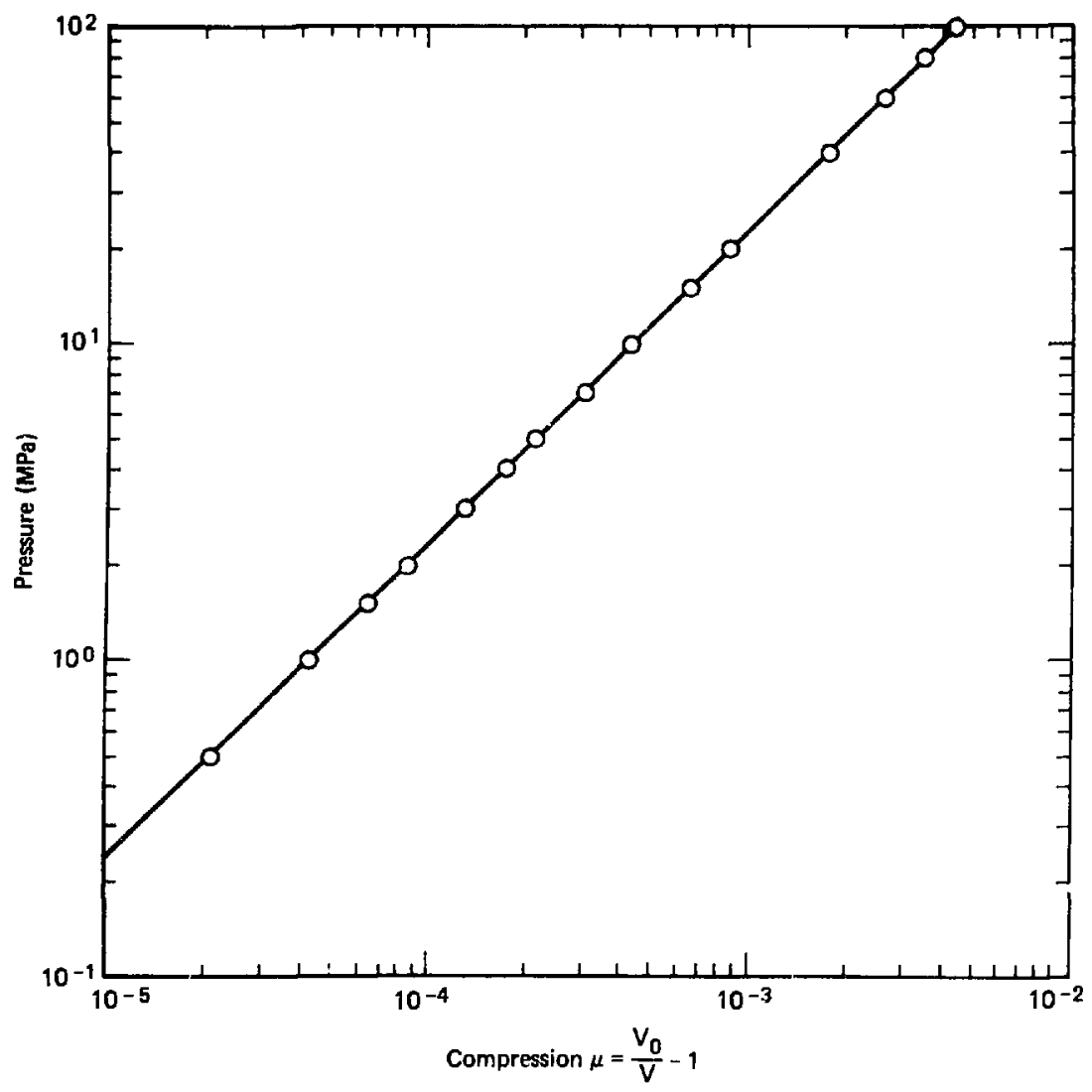

FIG. 7. Compressibility curve for Salmon salt, used as input for soc calculations. 
TABLE 1. Input parameter values used in SOC calculations in this report. Also shown are the corresponding values used by others in their calculations.

\begin{tabular}{|c|c|c|c|c|}
\hline & $\begin{array}{l}\text { This } \\
\text { study }\end{array}$ & Rogers 14 & Patterson 15 & $\begin{array}{l}\text { Terhune, } \\
\text { Snel1, and } \\
\text { Rodean } 17\end{array}$ \\
\hline Medium & Salt & Salt & Salt & Granite \\
\hline Initial density $\left(\mathrm{g} / \mathrm{cm}^{3}\right)$ & 2.2 & 2.24 & 2.24 & 2.67 \\
\hline Elastic Poisson's ratio & 0.25 & & & 0.28 \\
\hline initial bulk modulus (GPa) & 23.18 & 29.0 & 32.42 & 46.84 \\
\hline Initial shear modulus ( $\mathrm{GPa}$ ) & 13.91 & 10.4 & 10.45 & 24.15 \\
\hline Sound speed $(\mathrm{m} / \mathrm{s})$ & 4357 & & & 5440 \\
\hline Total porosity & 0 & & & 0.0267 \\
\hline Saturation & $100 \%$ & & & $100 \%$ \\
\hline \multicolumn{5}{|l|}{ Shear strength ( $\mathrm{MPa}$ ) } \\
\hline Fast rising stress & $B$ & 50 & 40 & \multirow{2}{*}{$\begin{array}{l}\text { Dependent } \\
\text { on pressure }\end{array}$} \\
\hline Slowly rising stress & 3 & 14 & 14 & \\
\hline Tensile strength (MPa) & 0.2 & & & 5 \\
\hline $\begin{array}{l}\text { Energy, to melt medium } \\
M b\left(\mathrm{~cm}_{3} / \mathrm{g}\right)\end{array}$ & .0417 & & & .0493 \\
\hline $\begin{array}{l}\text { Energy to vaporize nedium } \\
\text { : }\left(\mathrm{cm}^{3} / \mathrm{g}\right)\end{array}$ & .1971 & & & .1767 \\
\hline Overburden pressure (MPa) & 18.1 & 18.1 & & 26 \\
\hline Mass of iron & $1151 \mathrm{~kg}$ & & $30001 b$ & $5910 \mathrm{~kg}$ \\
\hline
\end{tabular}

RESULTS OF THE BOC CALCULATIONS ON SALMON

We performed many 500 calculations, varying the compressibility and the shear strength curves for the salt. The arrival times for the elastic and the plastic waves $w$ re controlled by the shape of the compressibility curve, but the widths of the positive and negative phases of the velocity or the pressure curves were controlled primarily by the failure behavior.

For coupling studies, a quantity of primary interest is the residual displacement, so efforts were made to watch this quantity in the parameter studies. Most of the early calculations gave residual displacements that were too low, even with the salt with zero gas-filled porosity. As noted earlier, 
this difficulty may have been partly caused by the fact that the soc code did not allow for dilatancy upon shear failure.

The shear strength of the salt was found to have a strong effect on the residual displacement. Several different shear: failure curves were used in the SOC calculations. Figure 8 shows some of these curves, and Fig. 9 shows the resulting curves of residual tisplacement vs radial distance. The displacement curves for the 8-MPa and the 5-MPa shear strengths fit the experimental data moderateiy well. Figure 10 shows the computed values of residual RDP plotted as a function of shear strength.

Several velocity waveforms for the calculation where the shear strength was $8 \mathrm{MPa}$ are shown in Fig. 11, along with experimental results. The match is quite good, although the peak velocities of the calculated cirves fall below the peak valocities measured by Sandia. It may be that the artificial viscosity value used in the calculations attenuated and broadened the peaks to

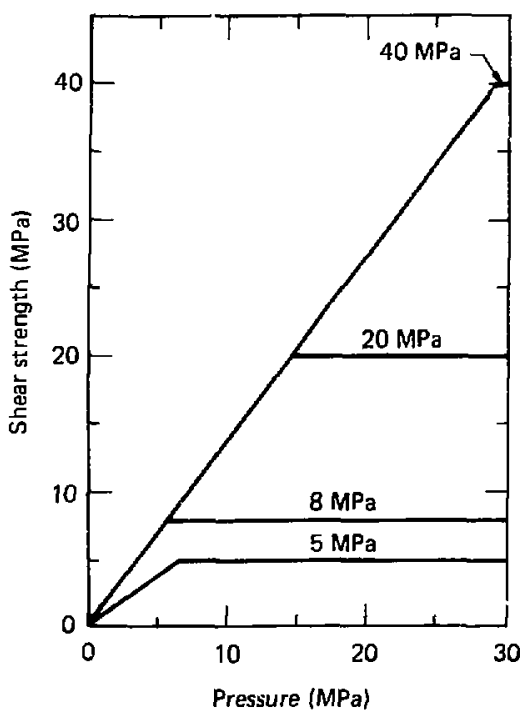

FIG. 8. Trial shear strength curves for salt as input for SOC calculations on the Salmon event.

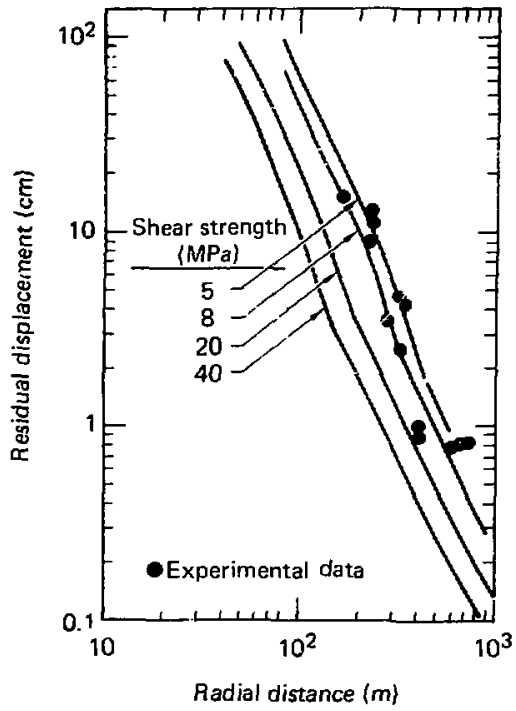

FIG. 9. Computed residual displacement plots for several shear strength values from SOC calcılations on the Salmon event. Data points are from the Salmon experiment. 


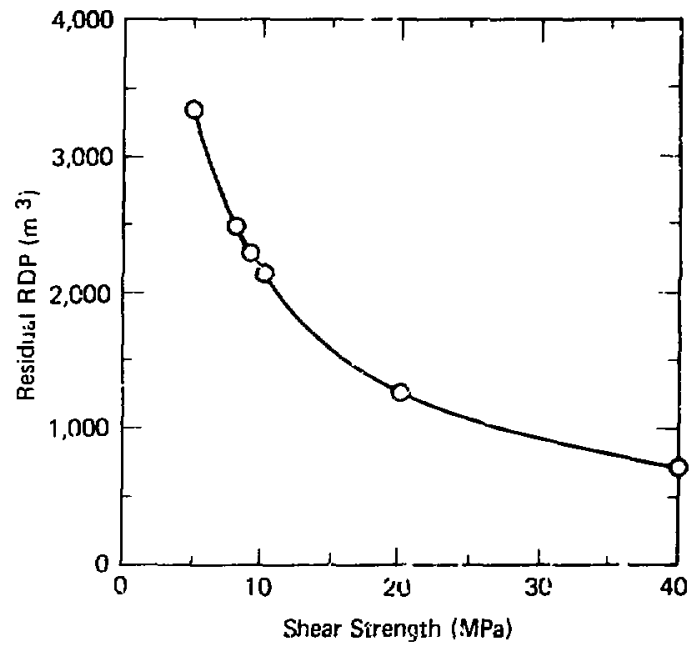

FIG. 10. Computed zesidual reduced displacement potential as a function of shear strength.

a greater extent than actually accurred. This form of attenuation in the calculation does not depend on the constitutive relations for the medium, but is a calculational artifact.

Figure 12 compares the displacement curves correspording to these velocity curves with the experimental curves. For these three curves, the calculated values lie below the experimental ones. There is a fair amount of scatter in the experimental points, as 1 s evident in Fig. 3, so a: exact match with all experimental data is not possible.

Ine cavity radii for several values of shear strength of the salt are given in Fig. 13, and the final cavity pressuris are shown in Fig. 14. It has been estimated that the Salmon cavity rafius was $22.3 \mathrm{~m}$ immediarely after shot time, and that the salt flowed plastically inwaid during the interval before the radius was actually measured several months later. ${ }^{27}$ This measured radius was $17.4 \mathrm{~m}$.

Figure 15 compares the curve of computed residual RDP for the 8-MPa shear strength with experimental values. Also shown is the RDP otsained from Eq. (6), for a cavity radius of $24 \mathrm{~m}$. Tatle 2 lists the $\mathrm{soc}$ resuits for the 

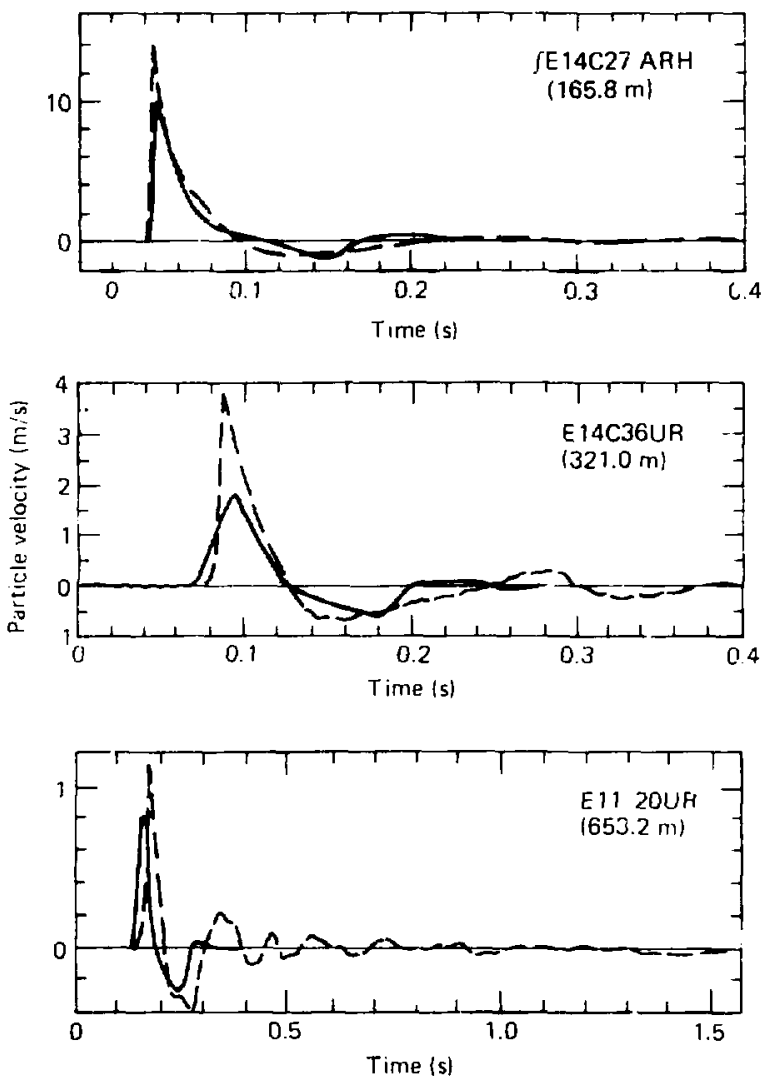

FIG. $1 \mathrm{l}$. Comparison of computed velocity histories with those measured by Sandia in the Salmon event. Solid lines wers obtained by caiculation; dotted lines represent experimental data. 

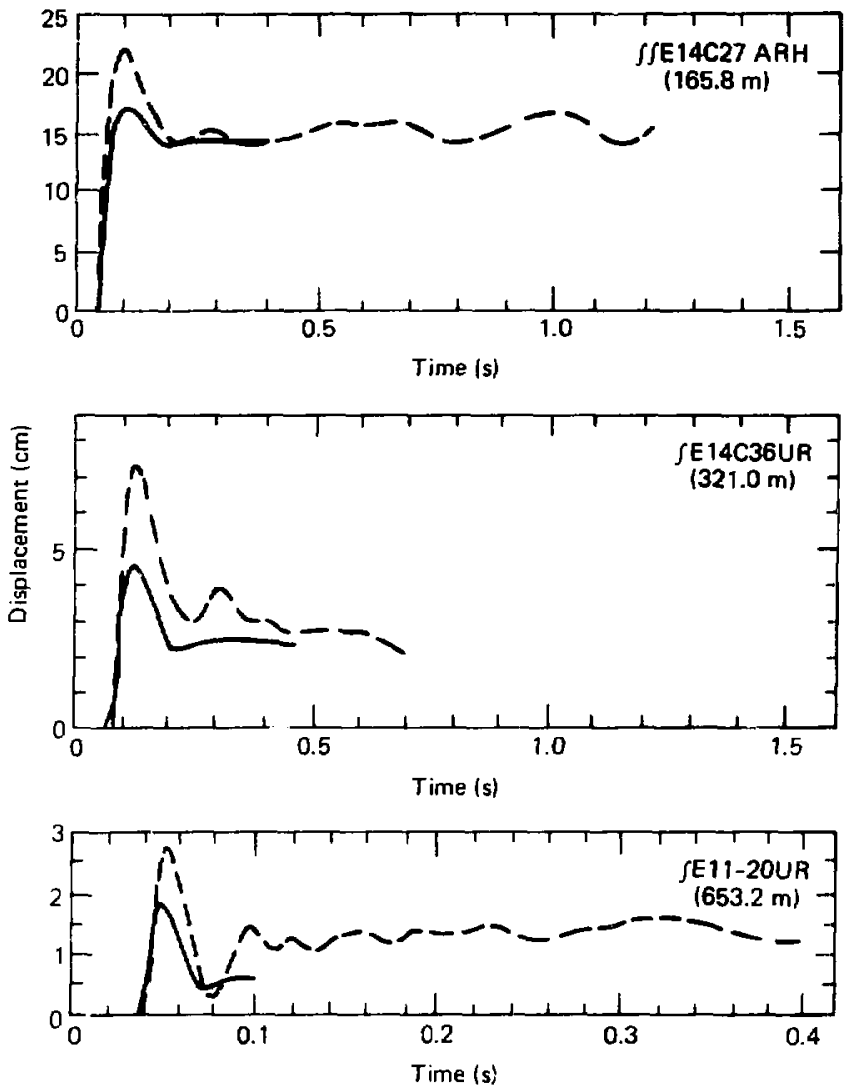

FIG. 12. Comparison of computed displacement histories with those measured by Sandia in the Salmon event. Solid lines were obtained by calculation; dotted lines represent experimental data. 


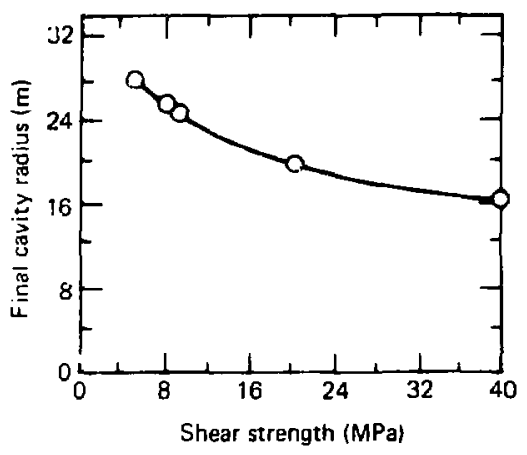

FIG. 13. Compuced final cavicy radius as a function of shear strength of the salt.

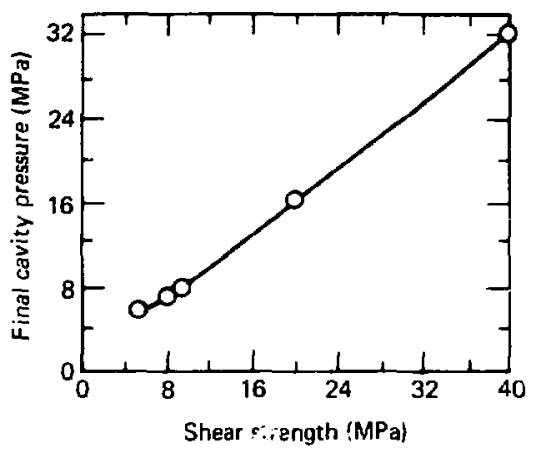

FiG. 14. Compuced final cavity pressure as a function of shear strength of the salt.

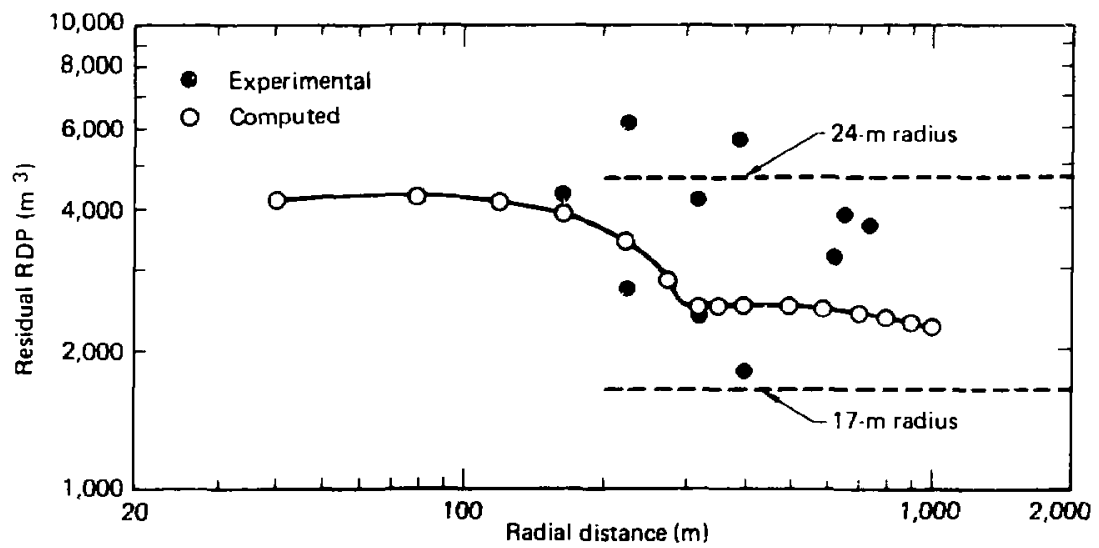

FIG. 15. Comparison of the computed residual RDF for Salmon with the experimental values obtained by Sandia. The dashed lines represenc the RDP for cavities of 17 - and $24-m$ radii. 


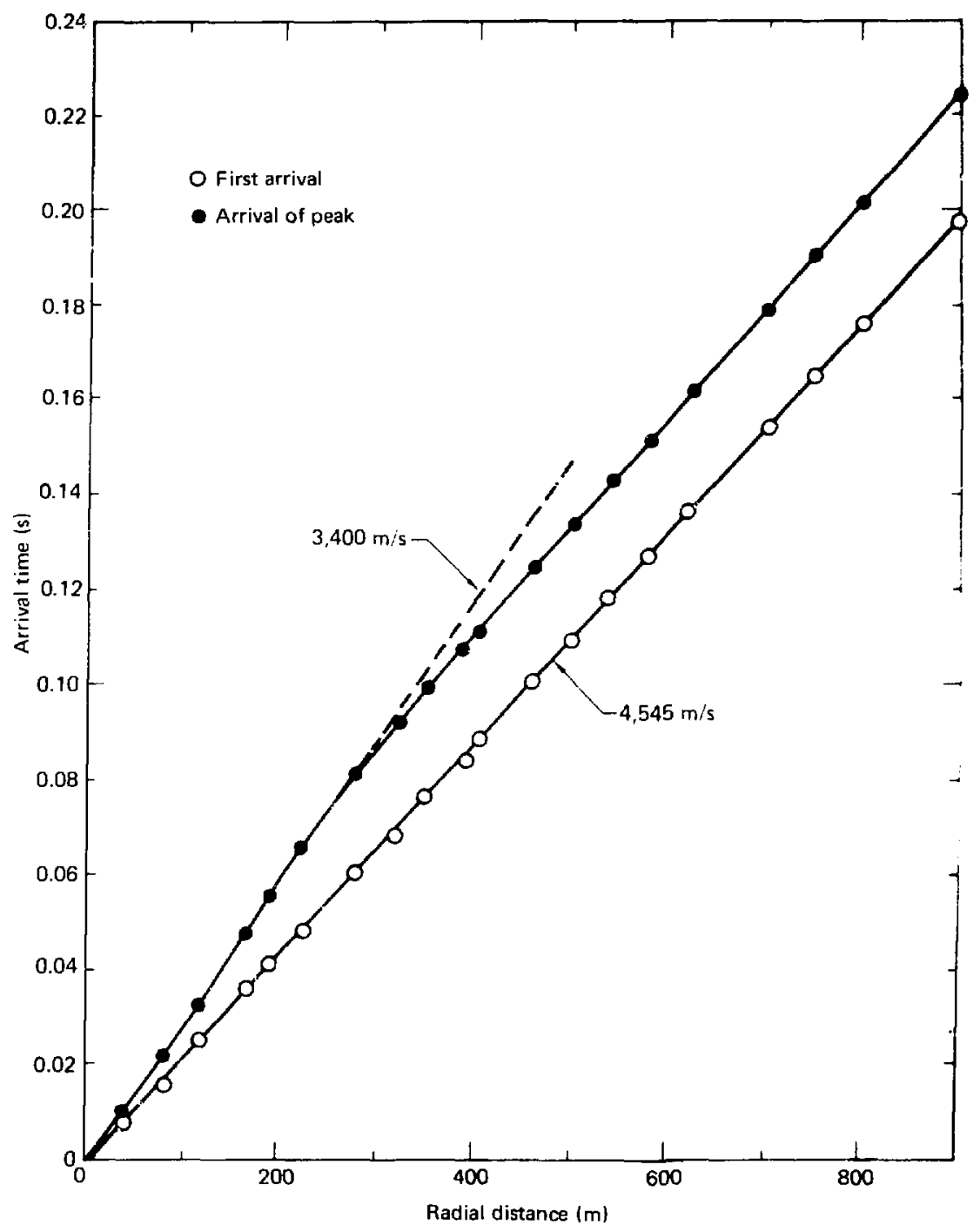

FIG. 16. Computed arrival-time curves for the leading edge and the peak of Lhe particle velocity waves for the salmon event. 
TABLE 2. Results of SOC calculations on explosions in Salmon salt with several values of shear strength. The energy source is $5.3 \mathrm{kt}$, overburden pressure $18.1 \mathrm{MPa}$, and initial cavity radius $0.5 \mathrm{~m}$.

\begin{tabular}{cccc}
$\begin{array}{c}\text { Shear strength } \\
\text { (MPa) }\end{array}$ & $\begin{array}{c}\text { Final cavity } \\
\text { pressure (MPa) }\end{array}$ & $\begin{array}{c}\text { Final cavity } \\
\text { radius }(\mathrm{m})\end{array}$ & $\begin{array}{c}\text { Residual reduced } \\
\text { displacement } \\
\text { potential }\left(\mathrm{m}^{3}\right)\end{array}$ \\
\hline 5 & 5.09 & 27.65 & 3350 \\
8 & 7.22 & 25.43 & 2490 \\
9 & 7.93 & 24.88 & 2280 \\
10 & 8.64 & 24.40 & 2130 \\
20 & 16.3 & 19.80 & 1260 \\
40 & 32.5 & 16.17 & 710 \\
\hline
\end{tabular}

effects of shear strength on final cavity pressure, final cavity radius, and residual RDP.

Figure 16 shows the arrival time curves for the elastic wave and the peak of the inelastic wave, as indicated by par-icle velocity measurements. These curves indicate wave velocities comparable to the experimentally measured curves of $\mathrm{Fig}$. 5, although the computed wave forms do not include che elastic precursor, and the first arrival of the plastic wave could not be identified in the computed waveforms. In the computed curve, the pulse peak at first had a velocity of about $3400 \mathrm{~m} / \mathrm{s}$, but at a distance of about $275 \mathrm{~m}$, its velocity rose to that of the elastic wave.

\section{DETERMINATION OF ELASTIC RADIUS}

The radius at which the transicion from inelastic to elastic behavior occurs is of interest in coupling studies. It can be regarded as a measure of the energy available to be radiated as seismic waves. Furthermore, it can be regarded as a scale factor for the frequency of the seismic signal emitted, particularly in specifying the "corner frequency." Several criteria can be used to define this radius, although these criceria are not necessarily independent of each other. These criteria are listed as follows: 
1. The radius at which the residual displacement begins to follow a $1 / r^{2}$ relation. (This requirement is necessary but not sufficient.) At higher Erequencies, the attenuation rate is perturbed by the presence of both induction and radiation components, however, so that at very large distances, the peak displacement follows a $1 / \mathrm{r}$ relation, while the residual displacement still follows the $1 / r^{2}$ relation.

2. The radius at which the reduced displacement potential or the reduced velocity potential attains a constant amplitude and waveform. This criterion is independent of induction component effects.

3. The radius at which the wave velocities of all components of the stress pulse become sonic.

4. The radius beyond which the medium remains undamaged or unaltered afcer the passage of the stress wave.

5. The radius beyond which the residual pressure equals overburden pressure after passage of the stress pulse.

6. The radius beyour which the energy flux carried by the radiation field wave through a solid angle integrated over time remains constant.

7. A radius defined by the frequency content of the seismic waves, for some assumed radial stress history at the elastic radius.

Several of these criteria have been applied to the Salmon event, with only partial success. One of the difficulties is that the inelastic-elastic transition is not well defined in the actual situation. For example, a seismic wave attenuation factor $Q$ is included in determining transmission of seismic waves to large distances, even though at these large distances the medium behavior is often regarded as elastic. other difficulties are the lack of vital pieces of information, and the experimental uncertainties in the data actually measured.

In the soc calculations the transition between the regions of inelat-tic . and elastic behavior is more clearly defined, although this transition is not necessarily realistic. Inelastic behavior occurs at some radius in SOC only where the stresses at that radius reach the failure envelope, either in shear or in tension. The phenomenon of compaction is not present in this nonporous medium.

The experimental data on Salmon and the results of the SOC calculations are evaluated here using these seven criteria for determining the elastic 
radius. The experimental data have too much scatter to determine the radius where the $1 / \mathrm{r}^{2}$ variation in residual displacement begins, as is seen in Fig. 3. In the computed plots of Fig. 9, the point at which $1 / \mathrm{r}^{2}$ behavior becomes evident is about $300 \mathrm{~m}$ for the 8 - MPa shear strength curve. Similarly, the field data do not indicate a radius where the RDP becomes constant, while tne calculations indicate a radius of $300 \mathrm{~m}$, as is evident in Fig. 15 .

In the experimental data in Fig. 5, it is not evident where the wave velocity becomes sonic, while the calculated curve in Fig. i6 indicates that this point is at $275 \mathrm{~m}$. With respect to damage of the medium, Rawson et a1. 21 have studied cores taken from a hole drilled inco the cavity several months after the Salmon shot, and found microfractures and other aiteration of the medium out to a radius of about $120 \mathrm{~m}$. I. the SOC results, the last zone in which damage to the medium had occurred was at 325 m radius.

There were no extensive pressure measurements made on the Salmon event. As seen in Fig. 17, the SOC results indicate that the uniform overburden pressure of $18.1 \mathrm{MPa}$ was reached at $325 \mathrm{~m}$ radius.

Perret has studied the sixth criterion, that of determining the integrated energy flux per unit area at several radiai distances for the Salmon, Sterling HE, Sterling, and Diode Tube events, all at the Tatum salt dome. 28 He evaluated the quantity

$$
F=\rho c \int_{t_{1}}^{t}[u(t)]^{2} d t
$$

where $\rho$ and $c$ are the density and the propagation velocity characteristics of the rock at the observing station, $u$ is free-field particle velocicy, and $t_{1}$ and $t_{2}$ are the times at which the motion began and ended. In Perret's calculations the total energy represented by the quantity $E=4 \mathrm{r}^{2} \mathrm{~F}$ did not reach a constant value out to the outermost measuring station, at a radial distance of $744 \mathrm{~m}$ for the Salmon event. For sterling and Diode Iube, it afnozrej tnat the ground motion was elastic already at the innermost station distances of $165.8 \mathrm{~m}$, and for Sterling $\mathrm{HE}$ at $261 \mathrm{~m}$. Perret did not include the effect of the deposition of the energy of the induction field component as permanent strain energy, however, so that his results are not completely valid. We did not perform the corresponding analysis of integrated energy flux on the $S O C$ results. As in the experimental case, the induction field 
components would be expected to make a simple direct integration of the total energy flux invalid for determining elastic radius.

Criterion 7 for determining elastic radius bears on the reason this radius is important in coupling studies. Over the region where the wave motion is inelastic, the concept of frequency components is not very useful because these components cannot te treated individually; the behavior of a single component depends on the forr of all the others. When the elastic radius is finally reached, these frequency components become independent, and wave motion beyond this point becomes calculable from the linear differential equations of stress wave motion in solid media. As a first approximation in some past analyses, the radial stress wave was assumed to have a step form at this elastic radius. A refinement was the addition of a spike, decaying away exponentially, superimposed on the step. 29-32

The linear differential equations for spherically symetric expansion have been solved by Sharpe ${ }^{33}$ and Blake 34 in complicated analytic form. Numerical solutions can be easily obtained with the use of a Fourier series solution, manipulated with the sockITTOME data processing code. 35 Simplified mathematical formulas for use with the Fast Fourier Transform (FFT) are developed in Appendix B. $1 \pi \mathrm{Fig} .18 \mathrm{ch}$ - scaled velocites at various scaled distances are sinown for a step radial stress input at the elastic radius. The velocity waveforms become narrower at larger radial distances until the effects of the induction term in the analysis become negligible.

The experimental measurements can be related to these calculated waveforms by a corparison of the time durations of the positive phases of the velocity curves. Perret ${ }^{18}$ has tabulated these values for the experimental Salmon data, as plotted in Fig. 19. There is a considerable spread in the values, with an indication that the durations at the larger radii were greater rather than less than those at smaller radii. These data indicate a duration of abuut $0.06 \mathrm{~s}$ at a distance of $300 \mathrm{~m}$. At a scaled distance of 2 on Fig. 18 , the dimensionless time duration is 1.5 . The time scaling factor is $t=$ $c t / a$, where $r$ is dimensionless $t i m e, c$ is sonic velocity, a is source radius, and $t$ is time. The source radius is then $a=c t / \tau=(4357)(.06) / 1.5$ $=174 \mathrm{~m}$. Obviously this estimate is quite crude.

The comparable time from the SOC run with an 8-MPa shear strength is $0.055 \mathrm{~s}$ at a radius of $300 \mathrm{~m}$. This leads to an elastic source radius of $167 \mathrm{~m}$. Healy, Chi-Yu King, and $U^{\prime} \mathrm{Neill}{ }^{36}$ have made a more thorough analysis on 


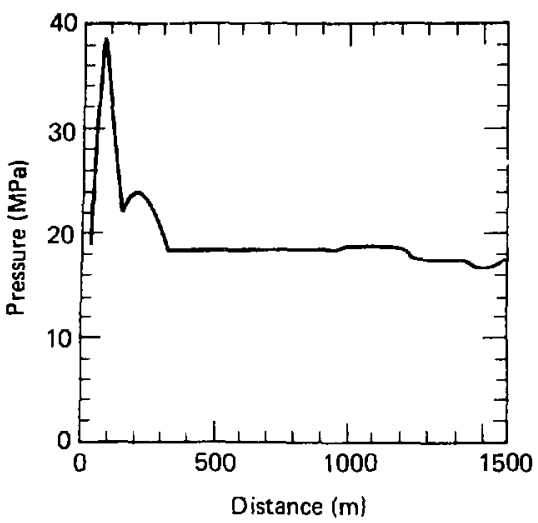

FIO, 17. Variation of residual pressure with distance at a late time, as indicated with soc. Elastic behavior is reached at a distance of $325 \mathrm{~m}$, where the pressure has returned to overburden pressure.

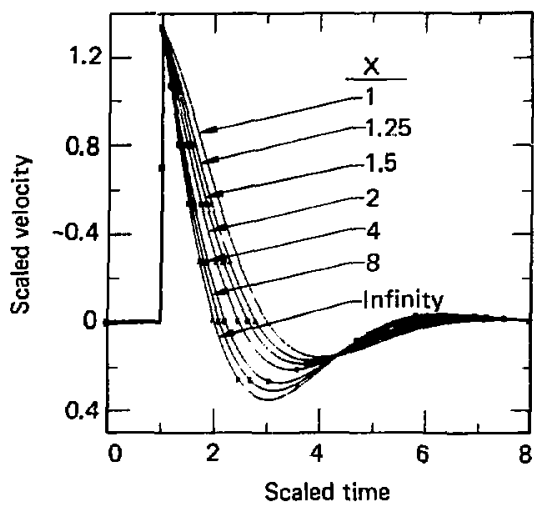

FIG. 18. Scaled velocity histories at various scaled distances for step pressure input, as calculated with the Fast Fourier Transform. A factor $1 / x$ has been removed from the curves for easier comparison, whare $x$ is scaled distance.

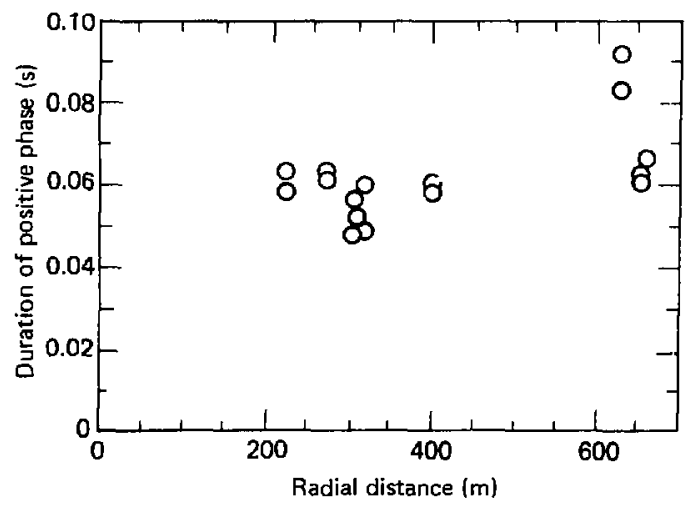

FIG. 19. Time durations of the positive phases of the particle velocity curves, as measured by Sandia in the Salmon event. (Adapted from Reference 18.) 
obtsining the apparent elastic source radius with a step input in radial stress from the rise time of the observed displacement. They used a pulse propagation velocity of $3960 \mathrm{~m} / \mathrm{s}$, and obtained an equivalent elastic radius of $169 \mathrm{~m}$.

Actually, the stress history at the inelastic-elastic interface is not a simple step in pressure for tamped explosions. Figure 20 shows the radial stress histories at several distances as calculated by soc. The region of elastic behavior is reached at about $300 \mathrm{~m}$ in these calculations, and at this distance the radial stress history consists of a broad spike, a trough, and then a stress plateau at a level somewhat higher than overburden stress. The residual pressure in the elastic region must be equal to overburden pressure, as is discussed in Appendix $C$.

When the region of elastic behavior is reached, the radial stress should remain unchanged in form at increasing distances except for the varying contributions of the induction and radiation components of the wave. This complication is avoided in the RVP plots, as shown in Fig. 21, wherc the RVP curves have reached an unchanging form at a distance of $319 \mathrm{~m}$.

Since the radial stress history at the elastic radius does not have a simple form, the usefulness of the elastic radius as a coupling indicator is diminished. At this elastic radius, however, the RDP-time and the RVP-time curves take forms that remain unchanged at all larger radii.

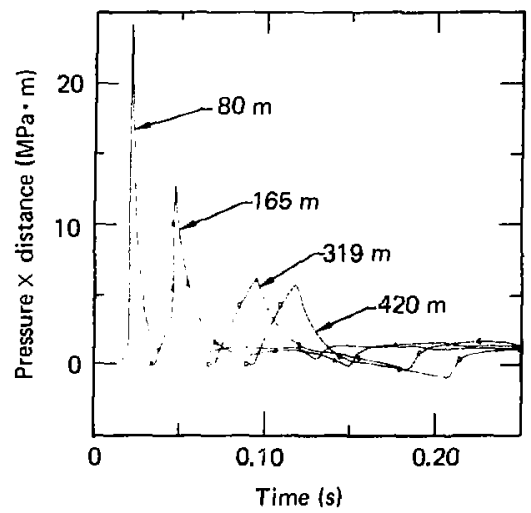

FIG. 20. Radial stress histories at several radial distances in the Salmon event, as computed with $\mathrm{SOC}$.

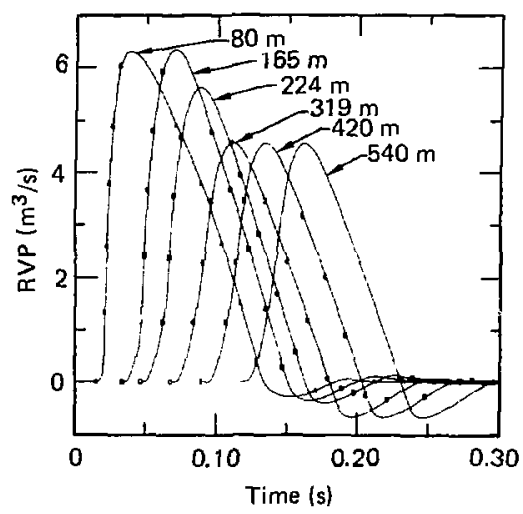

FIG. 21. RVP waveforins at several radial distances in the Salmon event, as computed with soc. 
It is concluded that the existing Salmon experimental data do not lead to a reliable determination of the distance out to the inelastic-ejistic interface. Instead, they indicate that the elastic state is approached in an asymptotic manner. Perret ${ }^{28}$ has observed elastic precursors at most of the accelerometer locations in the Salmon event, corresponding to radial stres; amplitudes up to $1 \mathrm{MPa}$, and merging into the main waves at transition stresses of about $1.95 \mathrm{MPa}$. If these precursors represent the elastic wave, then the main wave must involve energy loss to the medium, and the medium cannot be considered to be in the elastic domain.

In the SOC calculations, the inelastic-elastic interface is at a radial distance of $325 \mathrm{~m}$, as indicated by several of the criteria, especially by the beginning of the plateau of residual pressure as shown in Fig. 17.

\section{DISCUSSION}

The results obtained in this optimization of the input values for the SOC calculations have given fair agreement with the experimental data. The scatter in the experimental data precludes an exact fit.

The data shown in Fig. 6 and the results of the parameter optimization in the series of SOC calculations indicate that the Tatum salt failure was of the von Mises type--the failure appeared to occur at a particular value of shear stress, independent of the confining pressure. This salt behavior differs markedly from a material such as granite, where the shear strength is of a Coulamb-Mohr type, with strong dependence of shear strength on confining pressure.

Leo Rogers made Soc calculations on Salmon at the time it was fired, and obtained results similar to those reported here. His input values for shear failure were also of the von Mises type, and included a 50-to-60-MPa shear strengtn for a fast-rising signal, and $10 \mathrm{MPa}$ for a slowly-rising signal. He used a separate shear-strength value of $5 \mathrm{MPa}$ for material that had already failed. The SOC code at the time of his calculati.uns difiered from the present $50 \mathrm{C}$ code in several respects. In this earlier version, two separate shear strengths dependent on the loading rate were provided for, but this feature was later remned because there were not enough time-dependent failure data available to justify inclusion of this complication.

The same shear strength curve was used in the present calculations for the unfailed and the failed salt because of the requirements imposed by the 
relaxation option. Because of the plasticity of the salt, we believe that this limitation does not introduce gross error into the calculations.

The present version of SOC permits the use of compressibility and of shear strength data in tabular form. Actually, the compressibility curve used here was practically linear at pressures less than about $1 \mathrm{GPa}$, where the more important stress-strain interactions were occurring.

There is a large discrepancy between the values of shear strength of the Tatum salt of $32.6 \mathrm{MPa}$ as measured by the Bureau of Reclamation and the value of $8 \mathrm{MPa}$ as used in the SOC calculations. The following are some reasons for this discrepancy:

1. The medium may have been heterogeneous, with areas or planes of weakness larger in scale than the dimensions of the core samples. In this situation the strength tests would give higher values for shear strength than the values applitable for the SOC calculations.

2. The actual failure of the salt may have been complex, including inelastic yielding of some component of the salt medium at a shear stress corresponding to a radial stress of approximately 2 MPa, and gradually mare failure with increasing shear stress up to the laboratory failure value of $32.6 \mathrm{MPa}$. It is conceivable that for the soc input, a single failure stress value intermediate between shese two levels might give a fair match with the observed results.

It may be that both of these possible factors, as well as others not suggested here, account for the discrepancy between the Bureau of Reclamation value of $32.6 \mathrm{MPa}$ and the Soc input value of $8 \mathrm{MPa}$ for the shear strength of the salt.

DECOUPLING CALCULATIONS

GENERAL DESCRIPTION

All the foregaing calculations were made on the tamped Salmon explosion. These results were then used as a basis for further calculations on decoupled events. A reference energy source of $1 \mathrm{kt}$ was selected, to make scaling up or down in size more convenient than with the 5.3-kt energy source in Salmon. The constitutive equations used for the salt were the optimized set obtained from the Salmon calculations, with the limitation that the shear failure 
surves should be the same for original and for failed salt, as required by the relaxation requirement discussed in "Relaxation Analysis," below. In this set of calculations, the l-kt energy source was introduceo uniformly throughout an iron vapor that filled mined cavities of various sizes, and the resulting displacements, RDP's, and RVP's were calculated with the soc code. This uniform energy deposition produced pressure histories at the cavity walls that were stepwise in form.

In a Later set of calculations, the geometry of the sources was introduced in a more detailed form, and the gas motions inside the cavities were calculated with SOC. These calculations showed that the pressure histories at a savity wall actually consisted of a spike followed by a reverberating pressure signal.

\section{RELAXATION ANALISIS}

When a cavity is mined out in a salt formation chat is in a state of uniform pressure and of zero shear stress, this stress state is altered. The radial stress at the cavity wall becomes completely relieved, while the uniform overbulen pressure at large distances remains unchanged.

The scress distributions existing in the medium outside the cavity where the medium remairs in the elastic range have been solved by Lamé. His relations for the radial and transverse scresses $\sigma_{1}$. and $o_{i}$ For tine case where the cavity pressure is zero and the distant overburden pressure is o are the following:

$$
\begin{aligned}
& \sigma_{r}=-P_{0}\left[1-(a / r)^{3}\right] \\
& \sigma_{t}=-P_{0}\left[1+\left(\frac{a / r}{2}\right)^{3}\right]
\end{aligned}
$$

The quantity a is the radius of the cavity. It is noted that the pressure, equal to $-\left(\sigma_{t}+2 \sigma_{t}\right) / 3$, is aqual to $P_{o}$ over this whole elastic region. The shear stress of can be found from Eqs. (10) and (11):

$$
\begin{aligned}
\sigma_{s} & =\frac{\sigma_{t}-\sigma_{r}}{2} \\
& =\frac{3}{4}\left(\frac{a}{r}\right)^{3} P_{0}
\end{aligned}
$$


This shear stress is a maximum at the cavity wall, where $r=a$. In the problem considered here the overburden pressure was $18.1 \mathrm{MPa}$, giving a maximum shear stress of $13.575 \mathrm{MPa}$. This is beyond the 8-Mra shear strength assumed for the salt, so local failure must have occurred and the stress distribution was not given by Eqs. (10) and (11), which are for an elastic medium.

The formulas tor the case where inelastic flow has occurred are given in Appendix $C$ for a particular Eailure curve spplicable to salt.

RELAXATION WITH THE SOC CODE

After the cieation of the cavity, the surrounding salt is no longer in a state of uniform hydrostatic pressure. The salt becomes prestressed to some degree, and failure from a subsequent explosion in the cavity depends upon this prestressing. It is necessary to assign the proper prestress values to each zone in finite difference computer calculations cofore the energy source is applied within the cavity for the dynamic calculation. Furthermore, if the salt is not in a stace of equilibrium before the detonation, it will relax during the dynamic phase of the calculation, so that the calculated effects will be the result from the superposition of the two different processes.

The static option in SOC has Deen used to relax configurations where the stresses from the overburden were comparable with the stresses expected from the later explosion in the cavity. Since this relaxation computation is quite slow, relatively small grids extending out only 400 or $600 \mathrm{~m}$ were used for these problems.

Figure 22 shows the resulting values of radiai and of cangential stress after relaxation of a geometry with a 20 -m-radius cavity, with an overburden pressure of 1B.l MPr, and with a shear strength of the salt of $8 \mathrm{MPa}$. Figure 23 shows the corresponding values of shear stress and of pressure. Also shown on these two figures are the values obtained from the analytic equations (C-7) and $(C-8)$ in Appendix $c$. The agreement is practically exact, indicating successful relaxation by the static option.

From Eq. $(\mathrm{C}-6)$, the elastic-inelastic interface was calculated to be at a radial Jistance of $25.23 \mathrm{~m}$, which agrees with the static results. Beyond this distance the stresses followed the Lamé relations for an elastic medium, and the pressure was constant, equal to overburden pressure. Within this distance the shear stress was constant, equal to the failure shear siress for the salt. 


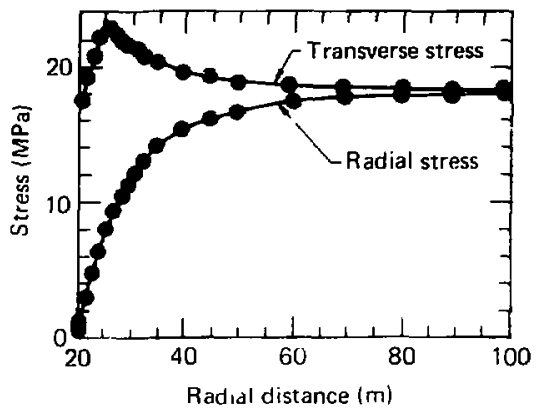

FIG. 22, Relaxed radial and transverse stressis around $a$ :nined cavity $20 \mathrm{~m}$ in radius. Shear strenzth was $\delta$ ypa and averburden pressure was 18.1 MPa. Solid lines are analytic surves; data points are from soc.

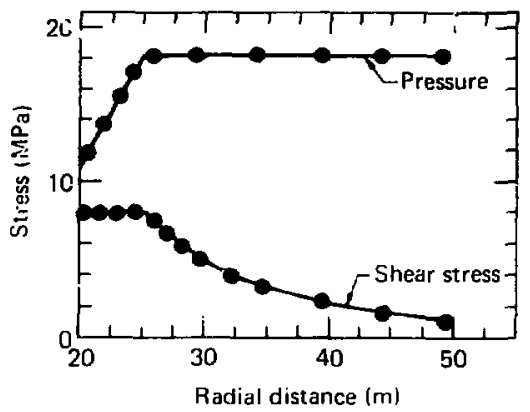

FIC. 23. Relaxed pressure and shear stress aruand a mined cavity $20 \mathrm{~m}$ in radius. Shear strength was $8 \mathrm{MPa}$ and overburden pressure was 18.1 spa. Solid lines are analytic curves; iata points are from SOC.

The stresses wert relaxed with the rather small inward tnotion of 1 . bi cm in this example. The numerical sesults obtained from Eq. (C-g) alsa gave an inward displacement ot i. X6 in at che cavity wall.

DYNAMIC GALEILATIAS WITH THE SOC CODE--STEP [NPITT PRESSURES

The Ivmanic calculations consisted essentially of it.serting $1 \mathrm{kt}$ of energy ints savities sf various radii in the salt, and letermining the resulting notions of the salt. For the problems where the cavity radius was 5 in or less, iL was nut acsessary to go through the static calculatiuns first, decause the stresses considered were trivial in comparison with the stresses resulting from the explosion. For cavities with radi of ln mor greater, a static phase was calculated first, to obtain an equilibrium stress state vefore initiation of the dynamic phase.

Decoupling san be calculated either by applying a fixed amounc of energy to cavities of various sizes, or by applying various anounts of energy to a cavity of a f:..ed size. Where relaxation was required, we used the second approach in most of the calculations presented here because it permitced us to calculace the preliminary explosion and subsequent relaxation only once for a series of decuupling calculations. The scaling required to convert to an equivalent injut energy of $l k t$ is discussed in Appendix D. The results 
obtained in the calculations described in this section were scaled back to an input energy of $l \mathrm{kt}$ in this manner. These scaling relations have bien verified in this study in that the resulting values of RDP and RVP es calculated by varying the cavity size or by varying the energy input in the SOC calculations fell on the same smooth curves.

The optimized input parameters listed in rable 1, obtained by matching with Salinon results, were used for these calculations. It was not realistic co include only the mass of the nuclear device in the larger cavitias, Decause tinis wuld leas to initial densities throughout the cavities far less than tnat of air at standard conditions. Instead, 6his ky, of izon were p'st lnto the cavity, and the air in the cavity, at a density of 0.001 g/cr, was added to the device assembly mass. The air was assigned the equation of state (Eus) ut the iron vapor in the calculations.

The calculation gridextender out to ad distance ot un! bou mi iul che runs where the cavity was large enoubl to necessilate relaxation. The uynanle calculations could be cairied au lo ouly o.lo s before interference oceureu

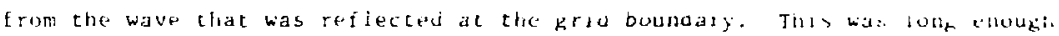

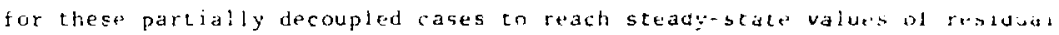
RDP. The peak values of RVP were reached varlie: in tame.

Figure 24 and Table 7 show the results for the residial kilp val ats it a

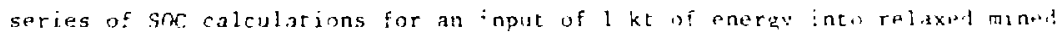

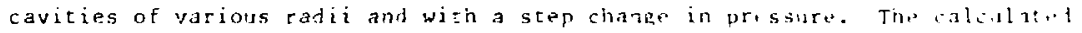
residual RDP was approximately $553 \mathrm{~m}^{3}$ for a cavity with a ra.i at i. m,

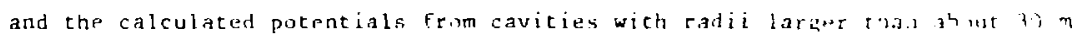
were approximately $3 \mathrm{~m}^{3}$. These galues indicaced a decolping rit. it abut lo4 for the low frequency componsut of the seismic waves.

The residual RDP obtained fom the salmon measurement

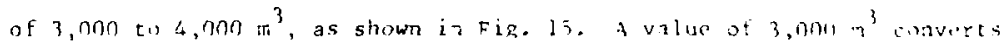

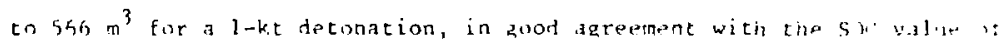
$553 \mathrm{~m}^{3}$ at a cavity radius oi n.s $\mathbf{n}$.

The calculateo static coupling increased appreciably is tat siae si the original emplacement cavity was increased from $0 . j m$ to $2-\eta$ in ratias. Tht coupling remained high out to about $10 \mathrm{~m}$, then decreased rapldy, and at a cavity radius of 20 in the explosion was alinost completelv decoupled for the induction field wave, as indicated by the rosidual RDP. 


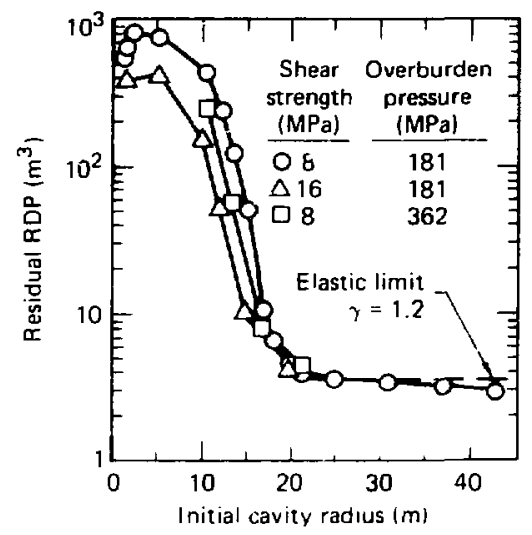

Flu. 2i. Variation of residual RDP with initial cavity radius as cumputeis with SOC in Salmon salt, for several values of shear strangth and overburden pressure, tor an input energy of $1 \mathrm{kt}$, and with step change in pressure.

The analve solution for residual RDp upon tho application it a itep in pressure on the walls if the cavity where the medium remains in its $-13, t$ range is:

$$
\psi(\infty)=\frac{P_{3}^{1}}{4 u}-
$$

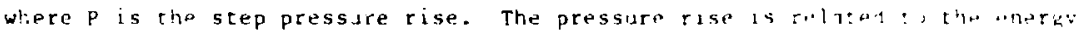
input through the relation

$$
P=\frac{(\gamma-1) F}{\frac{4}{3} \pi a^{3}}
$$

where $r$ is the ratio of enthalpy to internal energy, and 5 in the input energy. Fqs. (13) and (14) lead to the relation:

$$
\psi(\infty)=\frac{3(\gamma-1) E}{16 \pi \mu}
$$

It is noted that when all the medium around the cavity remans in the elast: range, the residual RDP is independent of cavity radius. 
TABLE 3. Calculated residual teduced displacement potentials, expressed in $\mathrm{m}^{3}$, as functions of cavity radius for an energy input of $1 \mathrm{kt}$. The four series have different values for shear strength and overburian pressure.

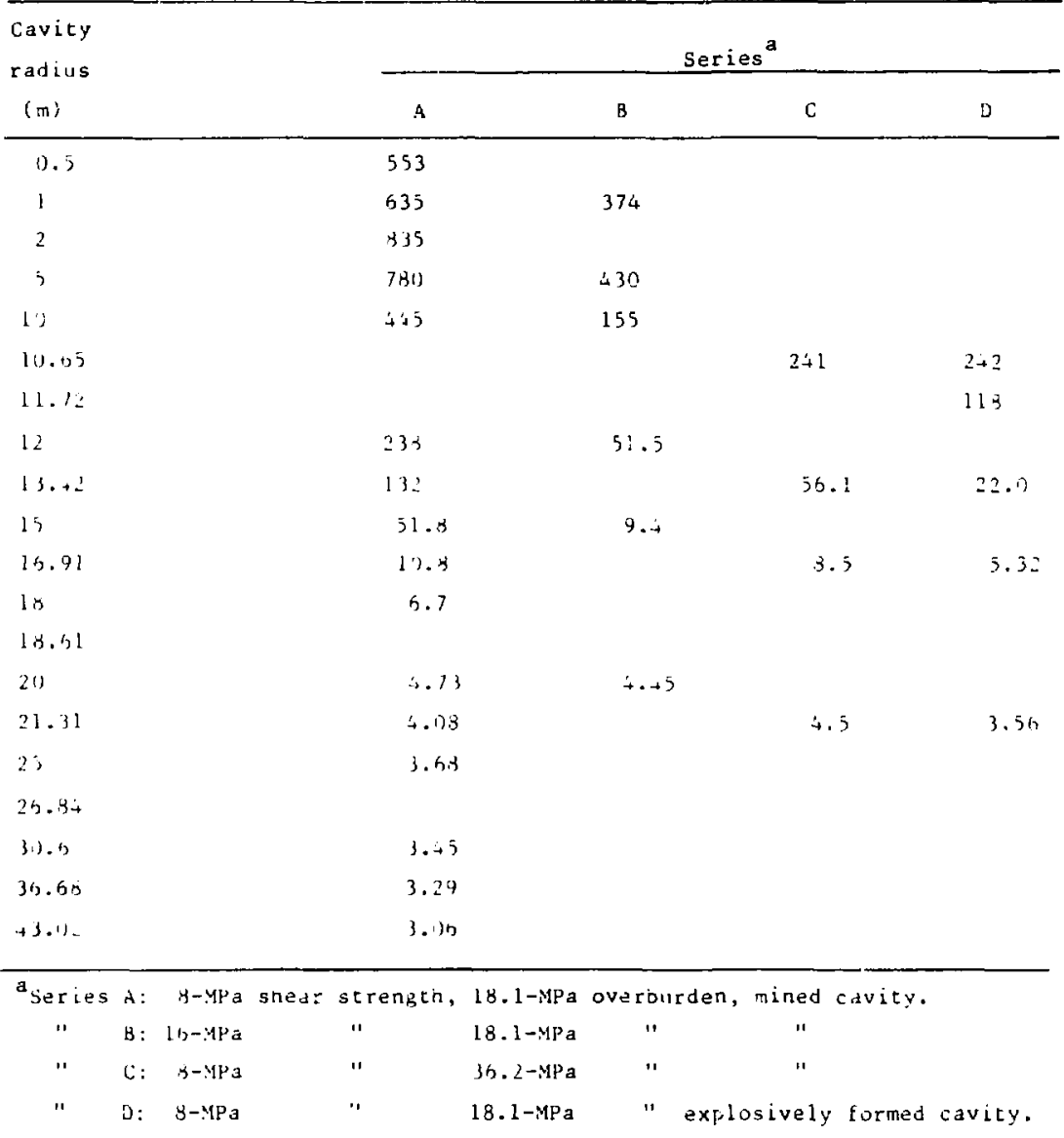


The appropriate value of $r$ is 1.2 , as will be shown later. For an input energy of $1 \mathrm{kt}$ and a value for $\mu$ of $13.9 \mathrm{GPa}$, the value for residual RDP from Eq. (15) is $3.6 \mathrm{~m}^{3}$. This value has been added to Fig. 24 for comparison with the points obtained from the SOC runs.

For cavities larger than $25 \mathrm{~m}$ in radius, the resulting energy density in the iron vapor was so low that the resulting values of $\gamma$ fell considerably below the value 1.2. Since in such cases most of the mass was that of the air in the cavity, and since the value of $\gamma$ for air should remain near 1.2 , corrections were made to these sor results. New values for energies corresponding to the step pressures in the cavity were calculated from Eq. (15), using a value for $Y$ of 1.2. The corrected values of cavity radius, RDP, and RVP were then scaled from these new energy values.

Even with this correction, the $50 C$ points beyond a $25-m$ cavity radius fell below the line reptesenting the theoretical elastic limit at the RDP value of $3.6 \mathrm{~m}^{3}$. The rcason for the discrepancy is not known.

The values calculated for peak RVP are Listed in Table 4 and plotted in Fig. 25, for $1 \mathrm{kt}$ of energy in relaxed mined cavities of various radii. The theoretical curve for peak RVP in the elastic region, as derived in Appendix E, has been added to his graph for comparison with the soc results.

This curve of peak RVP vs cavity radius, from the SOC calculations, has the same general form as that for the residual RDP (Fig. 24), with a region of high coupling at small cavity radii, and much lower coupling at larger cavity radii where the medium response is approximately elastic. There are several differences, however. In the elastic region the residual RDP becomes almost constant, while the peak RVP decreases as $1 / a$. At sinall cavity radii, the increase in residual RDP with increasing cavity size is quite large, while for peak RVy the increase is smaller.

A third difference is that the range in coupling indicatec by the residual KDP is greater than that indicated by peak RVP. Since the peak RVP curve does not reach a lower plateau at large radii, however, this comparison becomes subjective. The ratio of the residual RDP value from the cavity $2 \mathrm{~m}$ in radius to that from the 25 m cavity, where elastic behavior is apparenty reached, is 233 . The corresponding ratio for peak RVP for these two cavity sizes is 48.3 . This is a significant difference.

It is observed that the transition from high coupling to low coupling in the salt is not abrupt, but is rather gradual. Furthermore, the couplins, curves, such as those given in Figs. 24 and 25 , are plotted with respect to 
TABLE 4. Calculated peak reduced velocity potentials, expressed in $\mathrm{m}^{3} / \mathrm{s}$, as functions of cavity radius for an input energy of $1 \mathrm{kt}$. The four series have different values for shear strength and overburden pressure.

\begin{tabular}{|c|c|c|c|c|}
\hline \multirow{2}{*}{$\begin{array}{l}\text { Cavity } \\
\text { radius } \\
(m)\end{array}$} & \multicolumn{4}{|c|}{ Series $^{\mathbf{a}}$} \\
\hline & A & B & C & $\mathrm{D}$ \\
\hline 0.5 & 16,100 & & & \\
\hline 1 & 16,500 & 16,000 & & \\
\hline 2 & 28,500 & & & \\
\hline 5 & 26,000 & 14,000 & & \\
\hline 10 & 8,000 & 4,500 & & \\
\hline 10.65 & 6,746 & & 5,318 & 4,683 \\
\hline 11.72 & & & & 2,596 \\
\hline 12 & 4,500 & 1,950 & & \\
\hline 13.42 & & & 1,751 & 1,197 \\
\hline 15 & 1,550 & 830 & & \\
\hline 16.91 & 722 & & 715 & 550 \\
\hline 18 & $6 \cap 0$ & & & \\
\hline \multicolumn{5}{|l|}{18.61} \\
\hline 20 & 500 & 500 & & \\
\hline 21.71 & 454 & & 452 & 408 \\
\hline 25 & 359 & & & \\
\hline \multicolumn{5}{|l|}{26.84} \\
\hline 30.5 & 275 & 250 & & \\
\hline 36.58 & 219 & & & \\
\hline 43.02 & 172 & & & \\
\hline
\end{tabular}

aseries A: 8-MPa shear strength, 18.1-MPa overburden, mined cavitv.

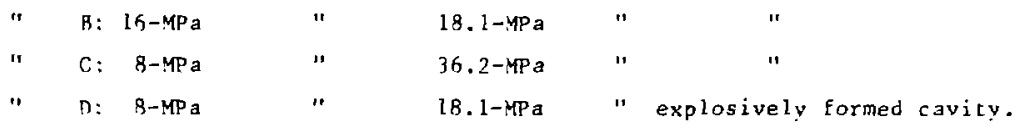




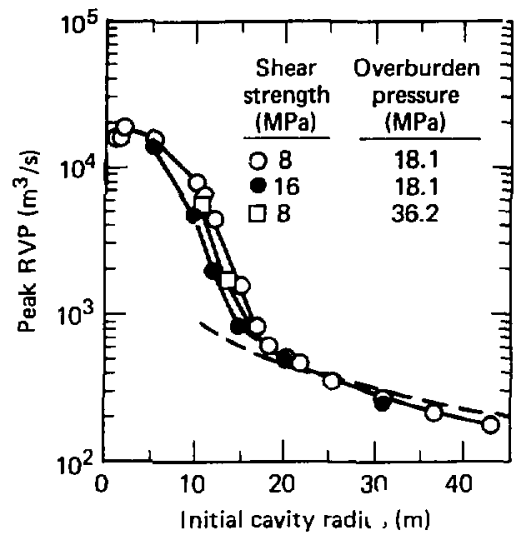

FIG. 25. Variation of peak RVP with initial cavity radius as computed with soc in salmon salt, for several values of shear strength and overburden pressure, and for an input energy of $1 \mathrm{kt}$. The dashed 1 ine represents theoretical peak RVP in an elastic medium.

cavity radius rather than volume. Since the energy density and pressure vary inversely with cavity volume, the volume parameter is more significant than the radius. The data of Figs. 24 and 25, for the 8-MPa shear strength and the 18.1-MPa overburden pressure with the mined cavity, are replotted as residual RLP versus cavity volume in Fig. 26 , and a.j peak RVP vs volume in Fig. 27 . These graphs show that most of the decoupling occurs at a relatively small cavity volune. The conclusion is that a cavity of moderate size will lead to a moderate amount of decoupling, but that for complete static decoupling, the cavity must be very much larger.

Since the increase in coupling is associated with inelastic motion of the medium, it would be expected that a medium with a greater shear strength would decouple down to smaller values of cavity radius. Several soc runs were made with a shear strength of $16 \mathrm{MPa}$ for the salt rather than the usual $8 \mathrm{MPa}$. The resulting values of residual and of peak RVP are also shown in Figs. 24 and 25. As expected, the 16-MPa points lie to the left of the 8-MPa curve, indicating that a smaller cavity in the stronger medium is sufficient for decoupling.

In addition, the maximum residual RDP value in the soupled region is lower for the stronger medium. This feature had already been studied in the Salmon investigation, where it was found necessary to use a low value of shear 

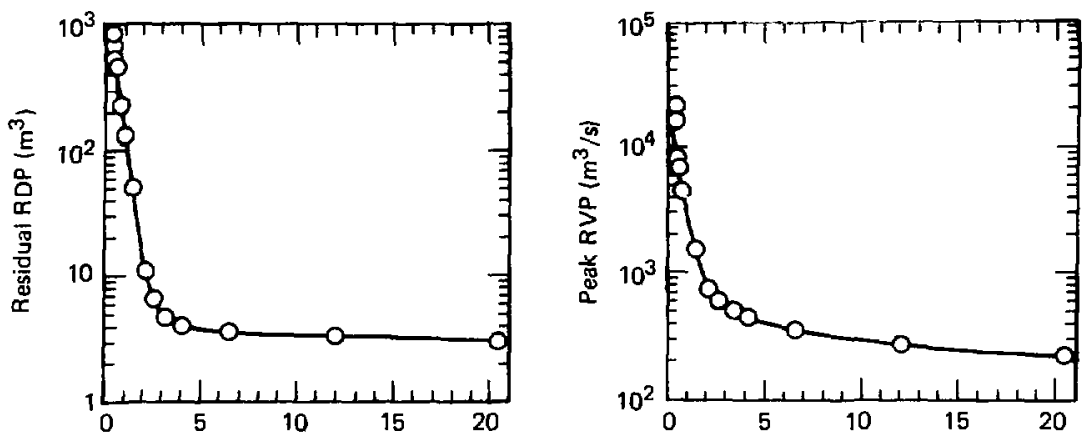

Initial cavity volume $\left(10^{4} \mathrm{~m}^{3}\right)$

FIG. 26. Variation of residual RDP with initial cavity volume for mined cavities, as computed with SOC. Shear strength was $8 \mathrm{MPa}$, overburden pressure $18.1 \mathrm{MPa}$, and input energy $1 \mathrm{kt}$.
FIG. 27. Variation of peak RVP with initial cavity volume for mined cavities, as computed wit، SOC. Shear strength was $8 \mathrm{MPa}$, overburden pressure $18.1 \mathrm{MPa}$, ant input energy $1 \mathrm{kt}$.

strengih in order to obtain large enough values of displacement and of RDP to match the experimental results.

Several SOC runs were made to investigate the effect of the overburden pressure on the coupling. The shear strength of the medium was set at $8.0 \mathrm{MPa}$ but the overburden pressure was doubled, to $36.2 \mathrm{MPa}$. The stresses around a mined cavity of radius $16.91 \mathrm{~m}$ were then relaxed under this greater overburden pressure. The resulting radial distance of failed material was $37.6 \mathrm{~m}$, in good agreement with the value of $37.3 \mathrm{~m}$ obtained from Eq. $(\mathrm{C}-6)$. The various values of energy were put into the cavity, and the results were scaled to give the residual RDP vs cavity-radius data, also shown in Fig. 24 , and the peak RVP vs cavicy-radius data in Fig. 25.

An upper limit of residual RDP as the shear strength approaches zero can be found. In such a case, the final residual pressure inside the cavity will equal the overburden pressure. The cavity radius can then be obtained from Eq. (14), again assuming a value of 1.2 for $Y$. At $1 \mathrm{kt}$ of energy input and a pressure of $18.1 \mathrm{MPa}$, the radius of the resulting cavity was found to be $22.3 \mathrm{~m}$. It is valid to use Eq. (6) to obtain the RDP in this application, and the resulting limiting value for RDP is $3,697 \mathrm{~m}^{3}$. This is considerably 
higher than the peak value of about $800 \mathrm{~m}^{3}$ for the medium with $8 \mathrm{MPa}$ of shear strength as obtained from SOC.

Figure 28 shows the input pressures obtained from SOC for the various cavity radii, with the usual overburden pressure of $18.1 \mathrm{MPa}$ and a shear strength of $8 \mathrm{MPa}$. These are to be compared with the pressures as given by Eq. (14), where the value used for $\gamma$ is 1.2. The agreement between the two sets of points is fairly close. (This really indicates that the $\gamma$ in the SOC gas tables for iron vapor was approximately 1.2.)

Also shown are the inal pressures in the cavities, as obtained from sOC. This curve shows that in a cavity with a radius of $1 \mathrm{~m}$, the final cavity pressure for a camped explosion was much lower than for the explosion in a cavity with an original radius of 12 or $15 \mathrm{~m}$.

Figure 29 shows the variation of final cavity radius with original cavity radius. The $1 \mathrm{kt}$ of energy created a cavity 15 to $17 \mathrm{~m}$ in radius for all

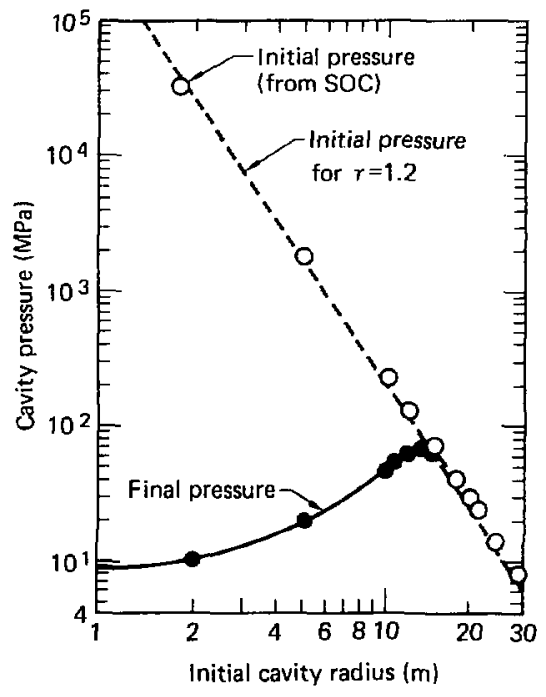

FIG. 28. Variation of initial and final cavity pressures with initial cavity radius, as computed with SOC. Shear strength was $8 \mathrm{MPa}$, overburden pressure $18.1 \mathrm{MPa}$, and input energy $1 \mathrm{kt}$. 


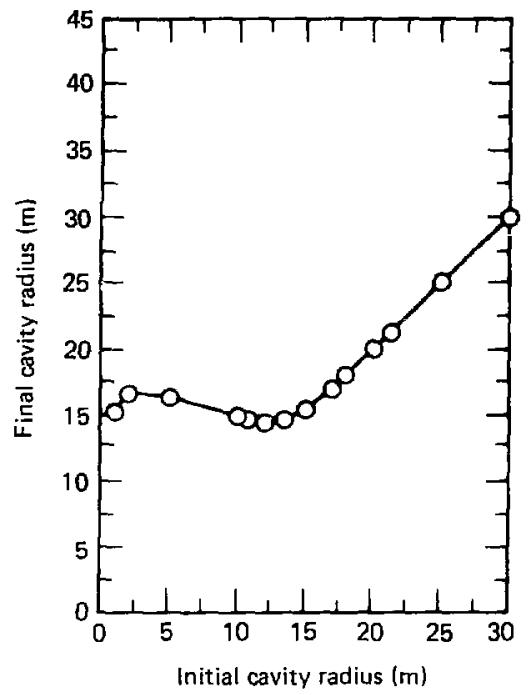

FIG. 29. Variation of final cavity radius with initial cavicy radius, as computed with SOC. Shear strength was $8 \mathrm{MPa}$, overburden pressure $18.1 \mathrm{APa}$, and input energy l kt.

origina! cavities chat were initially smaller. On the other hand, there was almost no increase in size for cavities that initially had radii larger than $15 \mathrm{~m}$. Table 51 ists chese data on cavity radii and pressures.

It is observed that an input of $1 \mathrm{kt}$ will create a cavity that is large enough to partially decouple a subsequent l-kt explosion in the same cavity. This sequence of shots involves complications that are considered in Section D.

COMPARISON WITH OTHER RESULTS

Haskel $1^{4}$ has calculated seismic coupling curves for a Coulomb-Mohr medium under the assumption of quasi-static expansion in response to an energy input inside the cavity. His shear failure curves for two values of internal friction coefficients are shown in Fig. 30 alang with those used by Terhune et al. 17 for granite, and with the failure curve used for the soc calculations 
TABLE 5. Calculated variation of final cavity radius, initial cavity pressure, and final cavity pressure with initial cavity radius. The energy source is $1 \mathrm{kt}$, overburden pressure is $18.1 \mathrm{MPa}$, and shear strength is $8 \mathrm{MPa}$.

\begin{tabular}{|c|c|c|c|}
\hline $\begin{array}{l}\text { Initial cavity } \\
\text { radius }^{a}(\mathrm{~m})\end{array}$ & $\begin{array}{c}\text { Final cavity } \\
\text { radius (m) }\end{array}$ & $\begin{array}{l}\text { Initial cavity } \\
\text { pressure (MPa) }\end{array}$ & $\begin{array}{l}\text { Final cavity } \\
\text { pressure (MPa) }\end{array}$ \\
\hline 1 & 15.15 & $3.42 \times 10^{5}$ & 8.79 \\
\hline 2 & 16.60 & $3.28 \times 10^{4}$ & 10.2 \\
\hline 5 & 16.36 & 1729 & 19.7 \\
\hline 10 & 14.86 & 216.6 & 48.0 \\
\hline 10.65 & 14.62 & 175.0 & 53.2 \\
\hline 12 & 14.45 & 122.6 & 62.5 \\
\hline 13.42 & 14.62 & 91.8 & 67.1 \\
\hline 15 & 15.40 & 68.7 & 62.3 \\
\hline 16.9 & 16.96 & 46.3 & 45.8 \\
\hline 18 & 18.04 & 38.4 & 38.2 \\
\hline 20 & 20.03 & 28.8 & 28.7 \\
\hline 21.3 & 21.3 & 23.0 & 23.0 \\
\hline 25 & 25 & 13.3 & 13.2 \\
\hline 30 & 30 & 7.0 & 7.0 \\
\hline
\end{tabular}

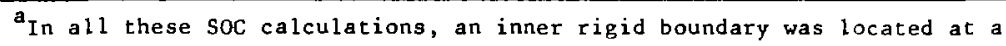
radial distance of $50 \mathrm{~cm}$ to prevent the occurrence of high transient pressures at the center.

presented in this report. Figure 31 shows the resulting RDP vs cavity radii curves that correspond to these failure curves. Haskell's curves were obtained for an overburden pressure of $5.1 \mathrm{MPa}$, and Terhune's curves were for $26 \mathrm{MPa}$.

It is seen from Fig. 30 that the input shear strength values used for the salt calculations presented here were lower than the Haskell values except for pressures lower than 30 or $50 \mathrm{MPa}$. It was not obvious what the effect of this difference in shear strength should be on the resulting RDP. Therefore we made a separate soc calculation to give results that could be compared more directly with Haskell's results. The data used by Haskel1, including an 


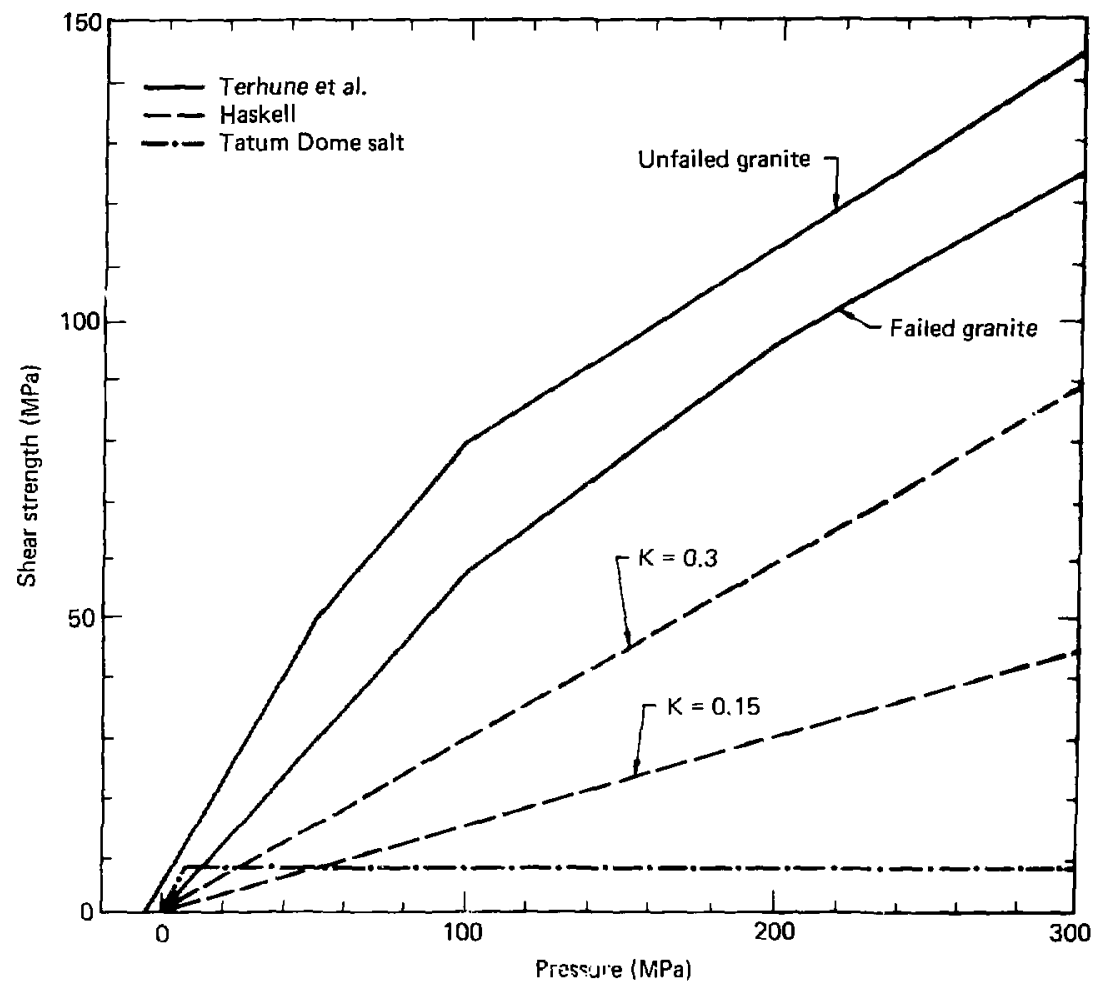

F[G. 30. Shear failure curves used by Haskel1,4 Terhune et al, 17 and in the calculations presented for Tatum dome salt in this report.

overburden pressure of $5.117 \mathrm{MPa}$ and a modulus of rigidity of $1.697 \times 10^{4}$ $M P a$, were used in the SOC input. We used the shear failure curve for $K=0.3$, shown in Fig. 30. The overburden stresses around the cavity were relaxed in che usual manner, using this shear failure curve, and the input energy values were then inserted into the cavity. Figure 31 shows two resulting residual RDP points from these calculations. It is seen that the agreement between these SOC points and the Haskell curve for $K=0.30$ is moderately good. 


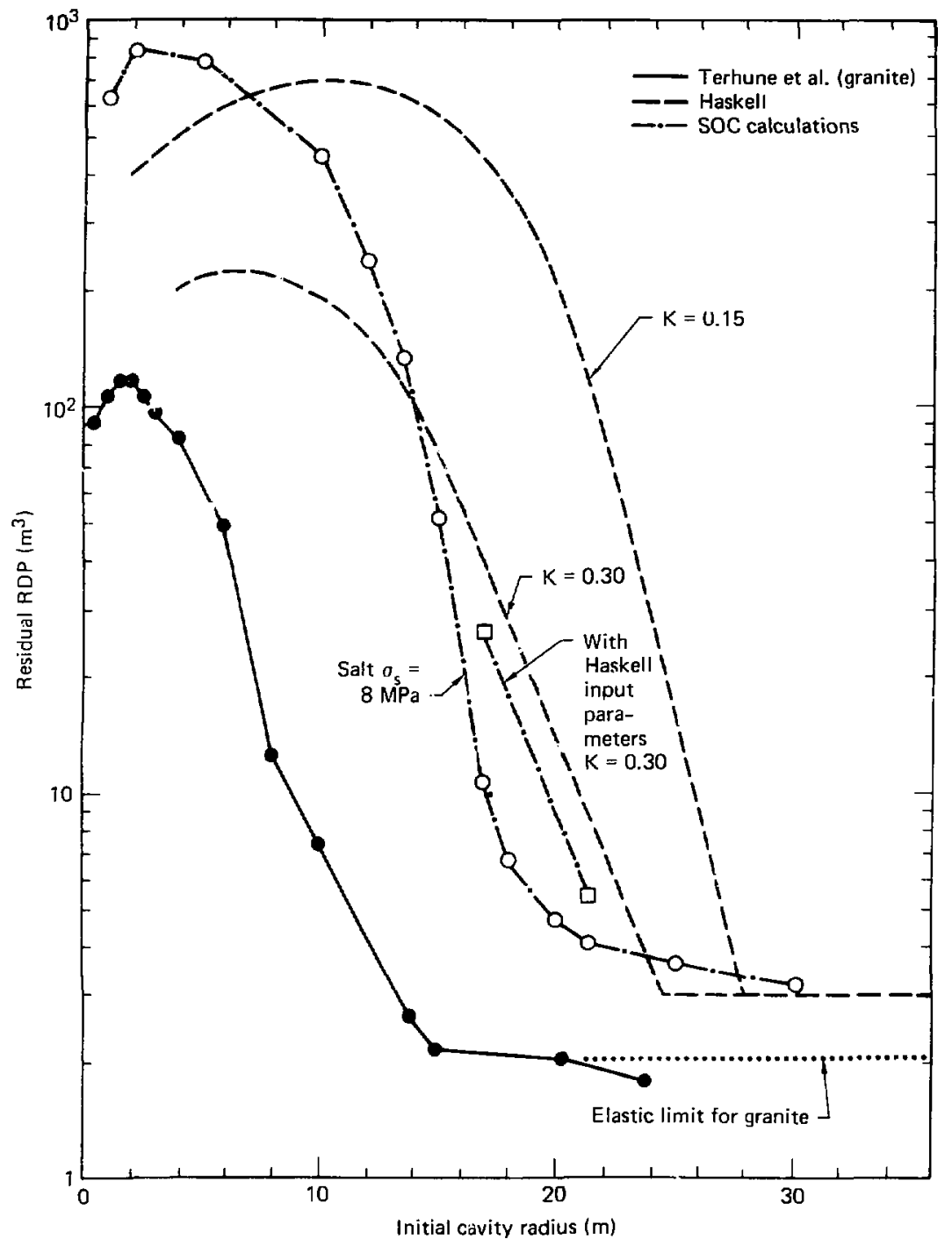

FIG. 31. Comparison between the curves of Haskel1,4 Terhune et al.,17 and the results of the SOC calculations presented in this report on the variation of residual RDP with initial cavity radius. Input energy was $1 \mathrm{kt}$. The shear failure daca used are as shoun in Fig. 30. 
DECOUPLING IN MINED AND IN EXPLOSIVELY FORMED CAVITIES

QUALITATIVE DESCPIPTION

The method of creation of an underground cavity has a strong effect on the stress field around the cavity. Furthermore, this pre-existing stress field would be expected to have an effect on the decoupling in these cavities. In the preceding section it was assumed that the cavity was a mined cavity, and the stresses were al lowed to relax inco their equilibrium configuration unjer the overburden piessure, with zero pressure inside the ravity. The energy from a nuclear ex.losion was then put into this cavity, and the restlting RDP was determined by a SOC calculation.

This procedure is not valid for a cavity seated by an earlier explosion because the earlier explosion will have produced its own non-uniform stress field. The material originaily inside the cavity has been moved outward, and this additional mass outside the cavity results in a region at high density and pressure. After this first explosion, a resifual pressure remains in the cavity, which is eventually vented to the atmosphere in normal prodedure. This pressure decrease is accompanied by a readjustment of the stresses in the surrounding medium. When a second explosion is fired in this cavity, the subsequent behavior of the medium depends upon this readjusted stress field.

This sequence of events was calculated with the soc code in the following steps:

(1) The cavity was first formed by application of 1 kt of energy at a point within the medium. Actually, the energy was applied to the inside of a small cavity $2.5 \mathrm{~m}$ in radius, representing a shot room, to permit larger zone sizes rear the origin. The entire grid extended out to a radius of $600 \mathrm{~m}$, which represented a compromise between the large size desiced in the dynamic phase to give information at large distances and prevent the early arrival of reflection effects from the outer boundary, and the small size desired co permit complete stress relaxation in a reasoncble amount of computer $t$ ime. The $\operatorname{soc}$ code was operated in the dynamic mode for this calculation.

(2) The residual pressure in che cavity was set to zero, corresponding to a release of the cavity gas to the atmosphere. The soc code was then operated in the static mode to relax the stressas in the medium to their new equilibrium configuration, under the 18.1-MPa overburden and with the additional mass present around the cavity. The stresses were considered to be 
completely relaxed when material velocities dropped to a very low value, and when the stresses no longer changed with time.

(3) The energy source for which the decoupling was to be determined was applied throughout the cavity, resulting in a step in pressure, and a dynamic soC run was made. The decoupling could be determined either by applying a fixed amount of energy to cavities of various sizes, or by applying various amounts of energy to a cavity of a fixed size. In the decoupling calculations of this section, the first approach was used. The scaling discussed in Appendix 4 was used to convert the results to those of l-kt explosions in cavities of various radii.

For the minc 1 cavity, step ( 1 ) of the procedure for the explosion-created cavity was eliminated. Instead, a geometry was set up that included a cavity with a radius of $16.9 \mathrm{~m}$, equal to that created by the $1-k t$ explosion. The stresses around this cavity, resulting from the condition of overburden pressure of $18.1 \mathrm{MPa}$ and zero pressure in the cavity, were then relaxed with a SOC run in the static mode. The energy sources of various sizes were then applied inside the cavity and dynamic soc runs were performed to determine the resulting couplings.

RESULTS OF SOC CALCULATIONS

The 1-kt explosion in the salt medium resulted in a calculated cavity diameter of $16.9 \mathrm{~m}$. Figure 32 shows the resulting radial and transverse stresses, and Fig. 33 shows the pressure and shear stress fields. For this calculation, where $1 \mathrm{kt}$ of energy was put inco the original cavicy with a radius of $2.5 \mathrm{~m}$, the final residual pressure was $11.2 \mathrm{MPa}$. This value is less than the overburden pressure of $18.1 \mathrm{MPa}$.

These stresses were then relaxed with the SOC code, to the condition of zero cavicy pressure and $18.1 \mathrm{MPa}$ overburden pressures. The resulting stress ano pressure distributions are also shown in Figs. 32 and 33 . It is observed that this scress relaxation procedure changed the strain field very litcle from its earlier configuration. Only within about $50 \mathrm{~m}$ of radial distance were the stresses adjusted appreciably.

The stress field around this explosively created cavity is seen co be quite irregular. The irregularities in the $s 0 c$ output were creaced mainly by the shear failure of the salt medium as the first stress wave passed by and as the slower stress readjustments occurred. Some shear failure occurred out to 


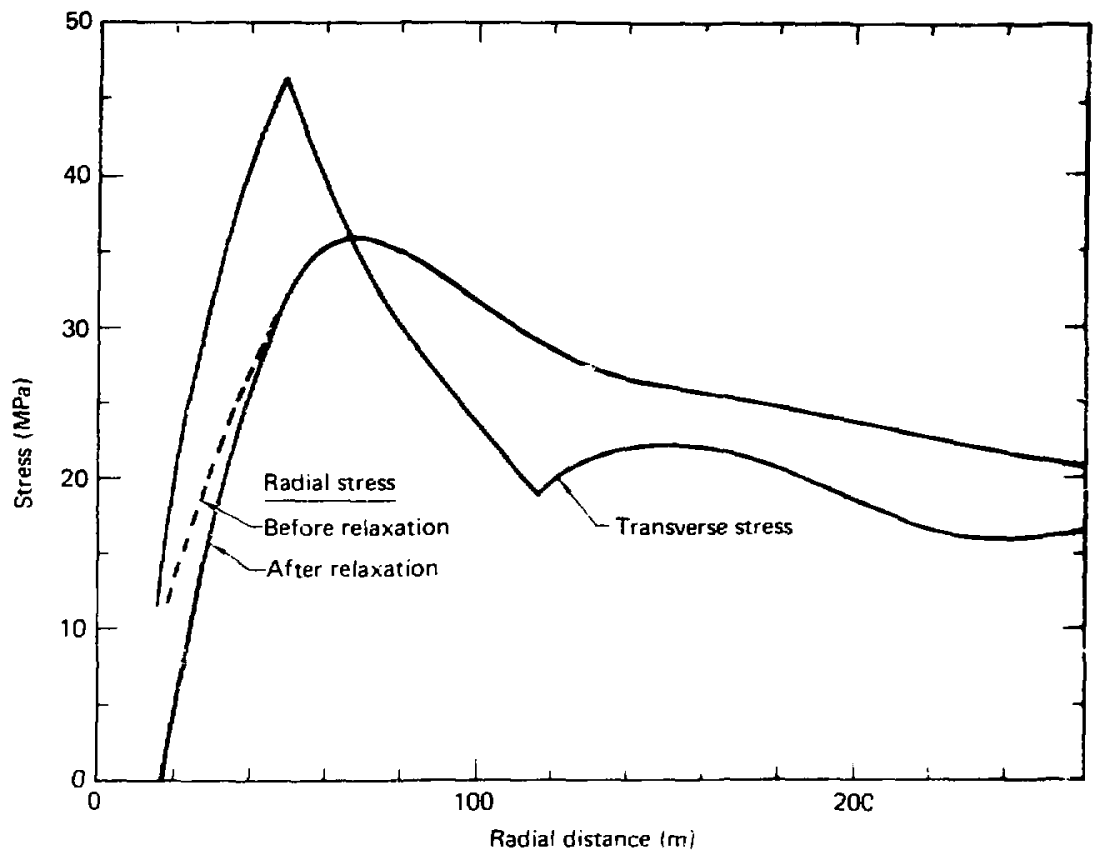

F[6. 32. Radial and transverse stresses around an explosively formed cavity $15.9 \mathrm{~m}$ in radius sefore and afcer stress relaxacion, as computed with SUC. Shear strength was $\mathrm{g}$ MPa and overburien pressure was $18.1 \mathrm{MPa}$.

a radial distance of $226 \mathrm{~m}$, as indicated by the damage tabulation $2 \mathrm{n}$ the $50 C$ output. The damage at such a large distance was very small, though, 'n comparison with the damage at smaller radial distances.

The stress fields calculated by soc are highly dependent on the particular features of the shear failure data used for the salt medium. These details are probably not accurate, and in addition the soc treatments of the failure mechanisms are probably in only approximate agreement with reality. Consequently, these stress distributions are probably only approximately correct.

On the other hand, these results are probably better than the results from analytic studies. Analyses by Haskel $1^{4}$ and by Lewis and Treiman ${ }^{5}$ deal with aspects of this problem, but assume that the stress field is applied 


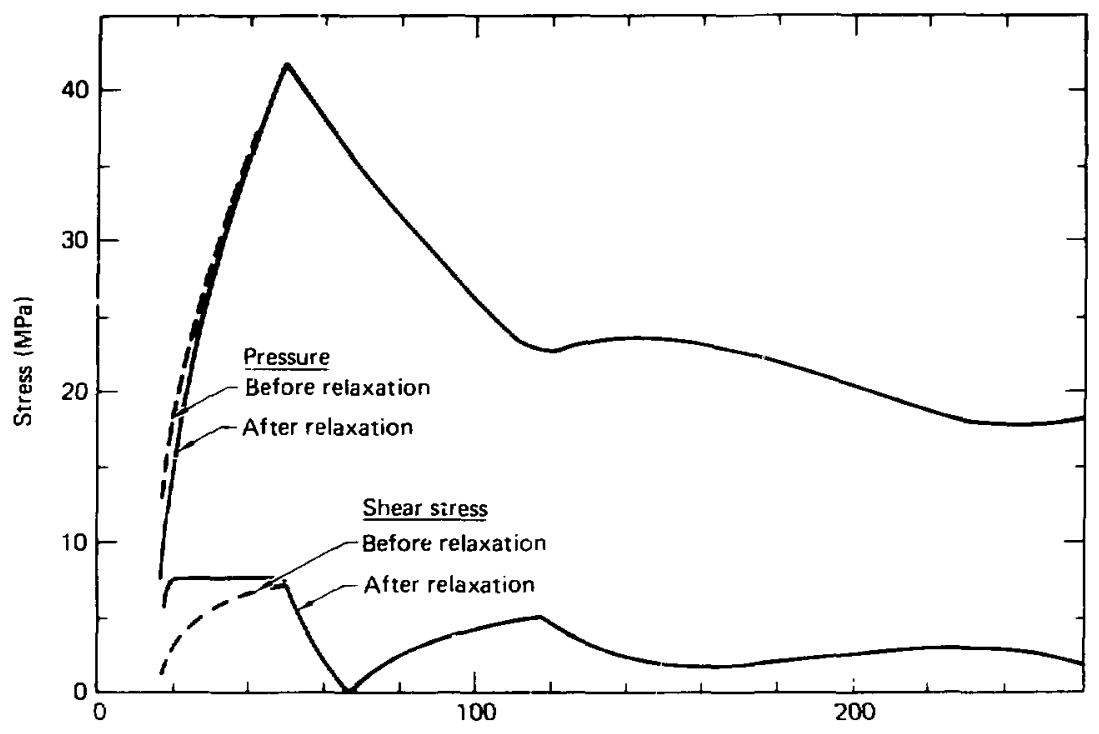

Radial distance $(\mathrm{m})$

F[G. 33. Pressure and shear stress around an explosively formed cavity 16.7 r. in radius before and after stress relaxation, as computed whth $S O C$. Shear strength was $3 \mathrm{MPa}$ and overburden pressure was $18.1 \mathrm{MPa}$.

slowly and adiabatically, so that the entire stress faeld as nisar mullbrtuin at all times. They to not consider the failure caused during the passage ui tre transient stress waves.

The pressure in the medium near the cavicy was increased when the explosively created cavity was formed because of the presence of the additional mass chat had been moved outward to form the cavicy. In the sinear failure curve assumed for these soc calculations, the shear strength was independent of pressure above the knee of the $a_{f}-p$ shear failure curves, sc that this extra pressure should not have affected the shear failure in SOC results for a subsequent shot in the cavity.

Figure 34 shows the calculated values for residual RDP for the case of an expicsion in a cavicy that had been explosively formed, and Fig. 35 gives the companion curve for peak RVP. These values are campared with the curves for RDP and RVP from shots in mined cavities. A result is that at some particular 


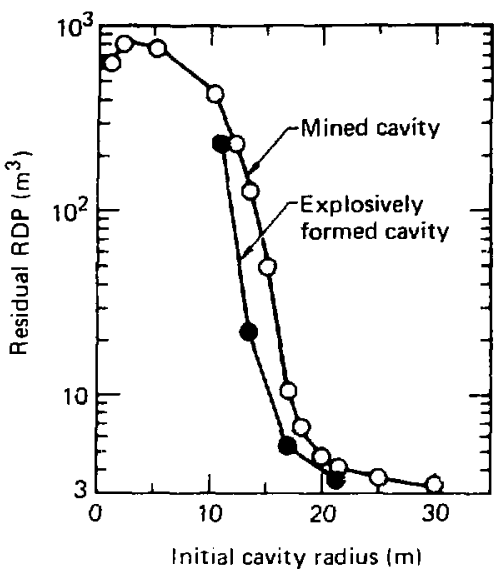

FIG. 34. Comparison of residual KDP for explosively furmed cavicies in Salmon salt with the RDP curve for mined cavities, as compuced with Soc. Input exergy was $1 \mathrm{kt}$.

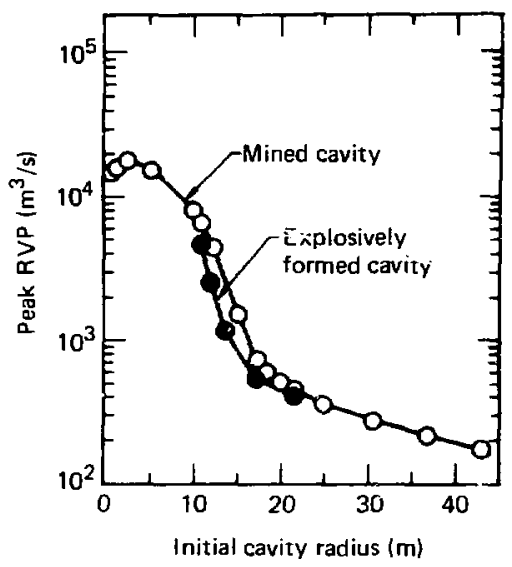

FIG. 35. Comparison of peak RVP for explosively formed cavities in salmon salt with the RDP curve for mined caviries, as computed with soc. Input energy was 1 ikt.

cavily radius, the resulting values of RDP and RVP for the explosion-formed cavity are less than those for the mined cavicy. The reason for this behavior may have been caused by the increased pressures in the medium near the cayity, resulting in an effect sinilar to that from increased overburden pressure. The suc calculations did not include strain-hardening effects.

DECOLPLING APPLICATIONS

COUPLING OF NUCLEAR AND CHEMICAL EXPLOSIONS

The energy density at the source for a nuclear explosion is very much higher than that from a chemical explosion, so that differences in seismic coupling inight occur. In the cowboy series of shots, the explosive used was Pefletol, with a density of $1.0 \mathrm{~g} / \mathrm{cc}$ and an output energy in a tamped configuration of $3200 \mathrm{joules} / \mathrm{gm}$. At this energy density, an explosive charge of l $\mathrm{kt}$ would fill a sphericai cavity $6.78 \mathrm{~m}$ in radius. This is not 
equivalent to a 1-kt nuclear explosion in a cavity of this radius because of the large amount of mass inside the cavity.

Comparative SOC calculations were made on 1 -kt nuclear and 1-kt chemical explosions in salt with the properties determined for the Salmon event, but at an overburden pressure of $5.33 \mathrm{MPa}$. The source radius for the nuclear calculation was $0.50 \mathrm{~m}$, while for the tamped chemical explosion it was $6.78 \mathrm{~m}$. The resulting values of residual RDP were $612 \mathrm{~m}^{3}$ for the nuclear shot and $1,283 \mathrm{~m}^{3}$ for the chemical explosion. The corresponding values of peak RUP were $17,200 \mathrm{~m}^{3} / \mathrm{s}$ and $27,850 \mathrm{~m}^{3} / \mathrm{s}$, respectively.

These results show that the seismic coupling from a tamped shor depends upon whether the energy source was nuclear or chemical. Consequencly, in the analysis of Cowboy, all calculations on decoupling were made with respect co a tamped chemical energy source, and in bribble, all were made with respect to the tamped Salmon event.

SUWBOY

Background

In Project Cowboy many experiments were carried out in which various charges of chemical explosives were deconated in cavities and camped holes in a salt medium. The masses of the charges varied fron 20 to $2,000 \mathrm{lb}$, and ground motions were measured out to $585 \mathrm{ft}$ from the explosions in free-field configurations. Surface seismic measurements were made out to $30 \mathrm{mi}$ from the source region, but useful records for the decoupled shots were obtained only at stations $14,400 \mathrm{ft}$ and $2:, 100 \mathrm{ft}$ from ground zero. Table 6 provides information on the various Cowboy shors.

Computer calculations with the SOC code were made for most of the Cowboy shots. The salt was assumed to be similar to that in Salmon, so the same material properties were used as before, including a shear strength of $8 \mathrm{MPa}$ for the salt. In the course of the calculations, it developed that the calculaced decoupling ratios for some of the shots were larger than those observed experimentally. Further calculations were made at salt shear strengths of $4 \mathrm{MPa}$, discussed below, to obtain results more in agreement with experimental results. 
TABLE 6. Sumnary of shot data for Project Cowboy.

\begin{tabular}{ccl}
\hline Shot no. & Charge weight (1b) & \multicolumn{1}{c}{ Type of shot } \\
\hline 1 & 20 & Coupled, 45-ft hole \\
2 & 20 & Decoupled, 12-ft diam sphere \\
4 & 100 & Decoupled, 12-ft dian sphere \\
5 & 100 & Coupled, 45-ft hole \\
6 & 198.35 & Decoupled, 30-ft diam sphere \\
7 & 200.0 & Decoupled, 30-ft diam sphere \\
8 & 199.65 & Coupled, 110-ft hole \\
9 & 477.4 & Decoupled, 30-ft diam sphere \\
10 & 499.7 & Coupled, 110-ft hole \\
11 & 954.0 & Docoupled, 30-ft diam sphere \\
12 & 1003.0 & Coupled, 110-ft hole \\
13 & 929.0 & Decoupled, 12-ft diam sphere \\
14 & 987.6 & Coupled, 110-ft hole \\
15 & 992.4 & Decoupled, 12-ft diam sphere \\
16 & 936.2 & Coupled, 110-ft hole \\
17 & 199.5 & Coupled, 45-ft hole \\
\hline & 199.8 & Coupled, 45-ft hole
\end{tabular}

The Cowboy shots were not as deep as the Salmon event. The 6-ft and 15-ft radius cavities were at the ends of horizontal drifts from the main mine workings at a depth of about $800 \mathrm{ft}$ below the surface, while the tamped shots were at the bottoms of holes drilled downward from the mine floor, so these shot points were somewhat deeper. Since the existing overburden stresses were not accurately known, it was assumed for the Soc calculations that the overburden stress vould be proportional to depth, with the salmon overburden pressure as the reference value. This gave an overburden pressure for Cowboy of about $5.33 \mathrm{MPa}$. Separate $\mathrm{SOC}$ calculations were made with this smaller overourden pressure at cavity energy densities corresponding to some of the Cowboy shots, for comparison with the experimental data. As in the earlier calculations presented in the section, "Dynamic Calculations with the SOC code," above, this change in overburden pressure did not have a strong effect on the calculated values of peak RVP or displacement. 
The output energy from the Pelletol explosive used in the Cowboy shots is not known accurately. Calorimetric measurements at LLNL, as reported by Trulio, ${ }^{37}$ indicate that the energy output from tamped Pelletol at full densicy is about 3,200 joules per gram, while the untamped output from small charges is 2,500 joules per gram. These values were used in the SOC calculations. Other Pelletol calculational parameters were obtained from Finger and collins. ${ }^{38}$

\section{Tamped Cowboy Shots}

Figure 36 shows the peak displacemenis as measured by Murphey ${ }^{8}$ on various Cowboy tamped shots. Also shown in this figure are the peak displacements as calculated with SoC for input energies of 200, 500, and 1000 lb of Pelletol and with a shear strength of $8 \mathrm{MPa}$ for the salt. The agreement between calculated and measured displacements is fairly good, although the experimental displacements decay with distance more rapidly than the salculated values do. The slopes of the experimental curves of peak displacement vs radial distance are about -1.5 , while the slope of the computed curves is about -1.1 at the larger radial distances. In the radiation field (far field) region in an elastic medium, the peak displacement decays as $r^{-1}$. The higher rate of decay of the experimental peak displacements was probably caused by the attenuation of the higher frequency components by the salt medium. The higher decay rate in the computed curves was probably caused by the effects of the finite zone size and by the artificial viscosity, since the stresses involved at the larger distances were well within the failure envelope assumed for the salt.

If it is assumed that there was no controlling length or time factor, the experimental results can be scaled and combined into a single plot, as shown in Fig. 37. The Soc results are also plotted on this graph. The agreement between the experimental points and the computed curve is fairly good, although the experinental data show the greater decay rate, as mentioned earlier.

In principle, the decay rate caused by inelastic effects implies an e-folding decay distance, which will ordinarily not scale properly. On the other hand, if the attenuation varies directly with frequency, then this decay distance varies directly with time, which scales as $\mathrm{E}^{1 / 3}$, and in this case, scaling with the cube root of energy is valid. It is probable that in cowboy and in the laboratory experiments, the ranges considered were not large enough 


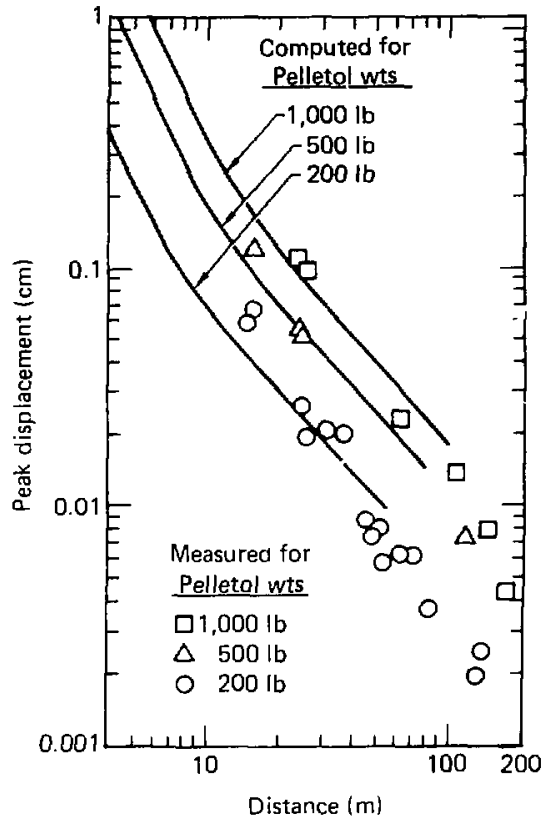

FIG. 36. Comparison of measured and computed peak displacements for Cowboy tamped explosions.

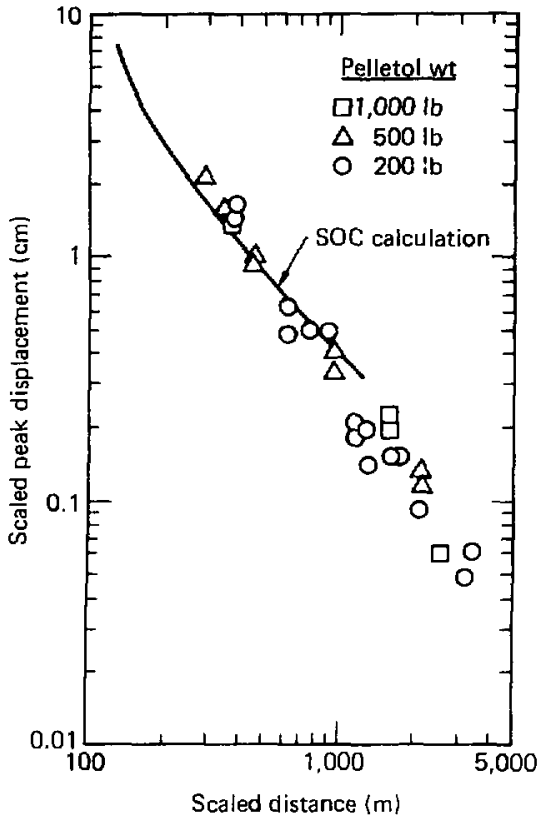

FIG. 37. Comparison of compured peak displacements with experimental data for Cowboy tamped explosions, scaled to an energy of $1 \mathrm{kt}$.

to permit the nonscaling of the inelastic attenuation to have a significant effect.

\section{Cavity Shots--Step Pressure Input}

In some of the Cowboy cavity shots, the expected shot pressuras were in the same range as the overburden pressures, so that the dynamic deformations were in the same range as the original static deformations that occurred before stress equilibrium around the cavity was reached. Because of this, in the calculations the stresses around the cavities were first relaxed to produce the condition of zero pressure within the cavity but overburden pressure at large distances from the cavity. Since overburden pressure was 
53.3 $\mathrm{MPa}$ while shear strength of the salt was $8 \mathrm{MPa}$, inelastic deformation in the salt around the cavity occurred before equilibrium could be attained.

In this relaxation process, the salt next to the cavity was unsupported in one direction, and some brittle failure might have occurred. Completely ductile behavior might be reached only at some distance from the cavity, where all the principal stresses were appreciably compressive. Heard, Abey, Bonner, and $D^{2} \mathrm{ba}^{23}$ have reported brittle failure when pure salt specimens were subjected to uniaxial stress, so it is plausjble that the impure Cowboy salt failed in a nonductile manner when subjected to stress in two directions. Because of this possibility, and because the experimental decoupling ratios seemed to require it, dynamic calculations were made for the cavity shors with a salt shear strength of $4 \mathrm{MPa}$, as well as a value of $8 \mathrm{MPa}$.

In the set of dynamic calculations, the energy was introduced uniformly throughout the cavicy gas, as described in "Decoupling Calculations," above. The amplitudes of the pressure steps for Cowboy shots 6,8 , and 10 were 0.19 ,

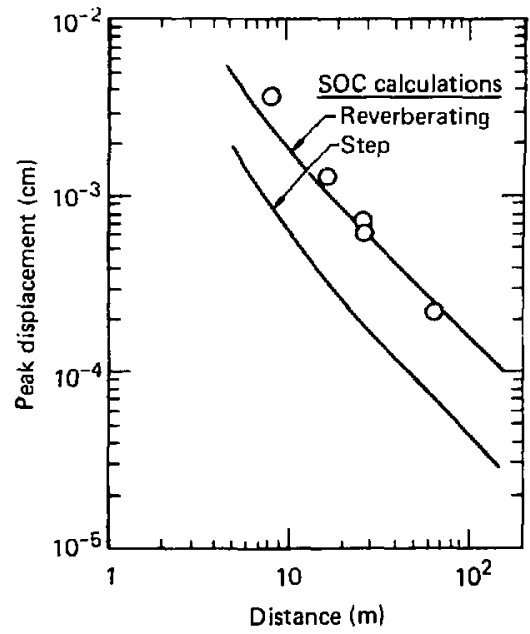

FIG. 38. Comparison of computed peak displacements with experimental data for Cowboy shot 6 . Charge weight was $200 \mathrm{lb}$ and cavity radius was $4.57 \mathrm{~m}$. Curves are shown for reverberating and for step covity pressure inpurs.

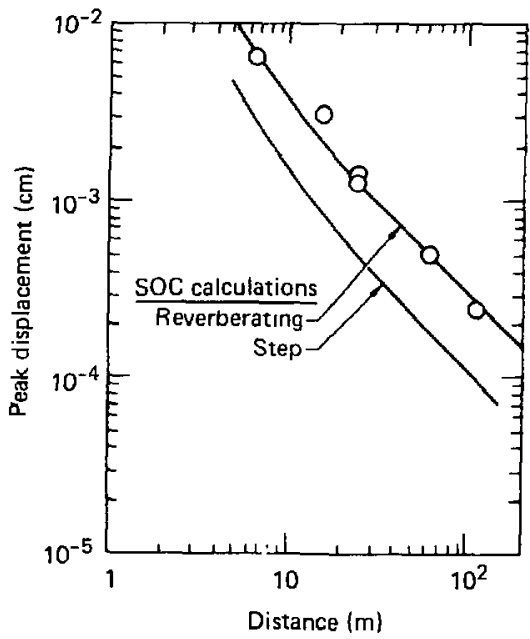

FIG. 39. Comparison of computed peak displacements with experimental data for Cowboy shot 8 . Charge weight was 477.4 ib and cavicy radius was $4.57 \mathrm{~m}$. Curves are shown for reverberating and for step cavity pressure inputs. 
0.46, and $0.92 \mathrm{MPa}$, respectively, as obtained from the energy-pressure relationships for air in the LLNL computer library and for Pelletol from the JWL ${ }^{38}$ tables. These pressure steps were considerably below the assumed shear strengths of 4 or $8 \mathrm{MPa}$ for the salt, so the salt should have behaved elastically with respect to this dynamic loading. Figures 38,39 , and 40 show the peak displacements calculated with FFT for Cowboy shots 6, 8, and 10 , respectively, and are compared with the experimental peak displacements as measured by Murphy. In every case the calculated values are below the experimental values by a factor of about three. These results do not depend upon whether the shear strength of the salt was 4 or 8 MPa because the salt remained in the elastic range for either strength value.

The calculated input step pressures for shots 12 and 14 in the 6-ft radius cavity were 14 and $29 \mathrm{MPa}$, respectively, so that inelastic deformation of the salt was expected, and it was necessary to use soC racher than FFT to calculate the salt motions. Figures 41 and 42 show the resulting calculated

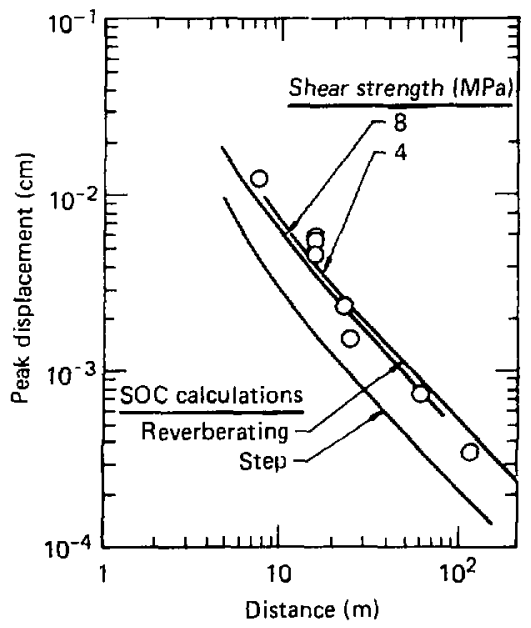

EIG. 40. Comparison of compuled peak displacements with experimental data for Cowboy shot 10. Charge weight was $954 \mathrm{lb}$ and cavity radius was $4.57 \mathrm{~m}$. Curves are shown for reverberating and for step cavity pressure inputs. 


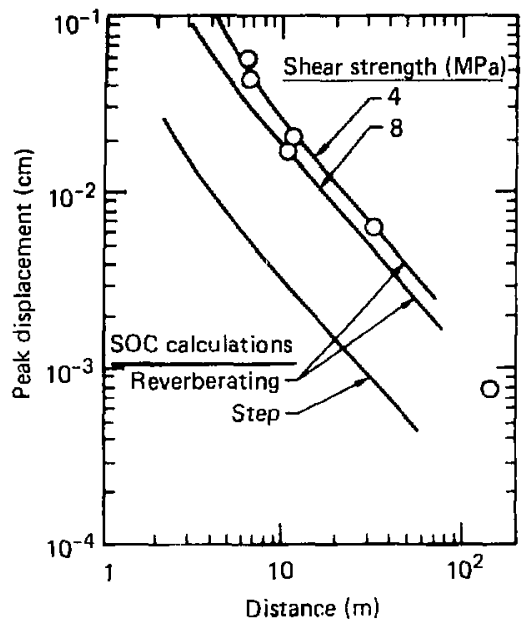

FIG. 41. Comparison of computed peak displacements with experimental data for Cowboy shot 12 . Charge weight was 929 lb and cavity radius was $1.33 \mathrm{~m}$. Curves are shown for reverberating and for step cavity pressure inputs.

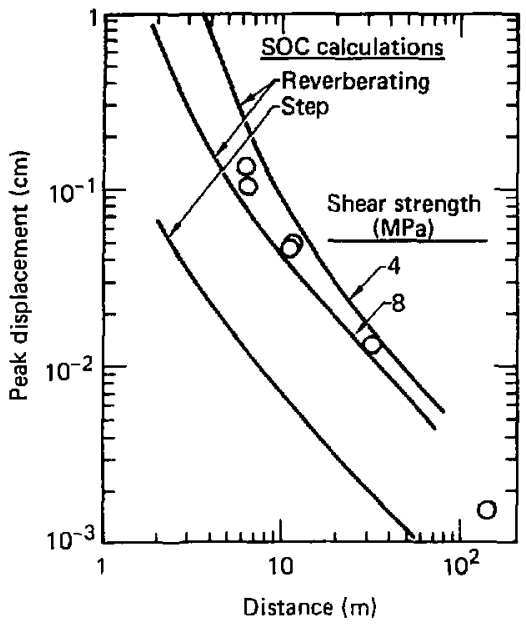

FIG. 42. Comparison of computed peak displacements with experimental data for Cowboy shot 14. Charge weight was 1902.4 lb and cavity radius was $1.83 \mathrm{~m}$. Curves are shown for reverberating and for step cavity pressure inputs.

displacements for the 8-MPa and 4-MPa shear strengths of the salt, and compare them with the experimental peak displacements. For these shots, the soc values lie below the experimental results by a factor of about five.

Since the experimental peak displacement curves for the cowboy decoupled shots did not reach the $\mathrm{r}^{-1}$ decay rate, they do not lead to unique values for peak RVP. Rough values may be obtained from the displacements measured at the 200-ft station for the 15-ft cavity shots and at the 100-ft station for the 6-te cavicy shots. The resulting values for peak RVP have been scaled to $1 \mathrm{kt}$, and are compared with the calculated peak RVP decoupling curve Erom Fig. 25 in Fig. 43. The experimental points lie considerably above the calculated curve.

The expcrimental decoupling curves for the Cowboy shots were determined by Herbst et al. 9 from comparisons of the seismic signals from pairs of shots of approximately the same charge weight, one tamped ard the other in a 


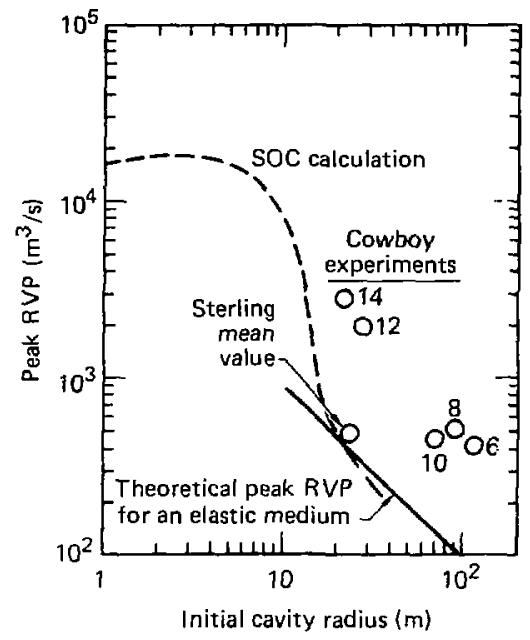

FIC. 43. Variation of computed peak RVP and experimental peak RVP with cavity radius, scaled to an input energy of $l \mathrm{kt}$, and with step pressure input. SOC calculation was performed using an 18,1-MPa overburden pressure and an 8-MPa shear strength.

cavity. Consequently, decoupling ratios were calculated with SOC on similar pairs of chemical explosions, so that the calculated and the experimental ratios could then be compared directly. Actually, since similarity conditions were met for the tamped chemical explosions, a single tamped pelletol shot was calculated, and values of residual RDP and peak RVP for various input energies were scaled from this single calculation by the relations given in Appendix $D$.

Table 7 lists the calculated values of residual RDP (or zero-frequency spectral amplicude of KVP), along with the calculated zero-frequency decoupling ratios for the cavity shots, with step pressure input. Also listed are the approximate values obtained Eron Herbst et al. " The racios are in rough agreement for Cowboy shots 6,8, and 10 , bist the computed decoupling ratios for shots 12 and 14 are much larger than those found from the seismic data. 
TABLE 7. Calculated pressures, values of residual RDP, and zero-frequency decoupling ratios for Cowboy and for Sterling.

\begin{tabular}{|c|c|c|c|c|c|c|c|c|c|}
\hline \multirow[b]{3}{*}{ Shot } & \multirow{3}{*}{$\begin{array}{l}\text { Cavity } \\
\text { radius } \\
\text { (m) }\end{array}$} & \multirow{3}{*}{$\begin{array}{l}\text { Input } \\
\text { energy } \\
\text { (J) }\end{array}$} & \multirow{3}{*}{$\begin{array}{l}\text { Input } \\
\text { pressure } \\
\text { (MPa) }\end{array}$} & \multicolumn{3}{|c|}{ Residual RDP ( $\left.{ }^{3}{ }^{3}\right)$} & \multirow{2}{*}{\multicolumn{3}{|c|}{$\begin{array}{l}\text { vecoupling ratio at } \\
\text { zero frequency }\end{array}$}} \\
\hline & & & & \multirow[b]{2}{*}{ Tamped } & \multicolumn{2}{|r|}{ Cavity } & & & \\
\hline & & & & & step & Reverberating & Step & Reverberating & Experimental \\
\hline Cowboy 6 & 4.572 & $2.268 \times 10^{8}$ & 0.19 & .0695 & .0032 & .0032 & 217 & 217 & \\
\hline Cowboy 8 & 4.572 & $5.414 \times 10^{8}$ & 0.45 & .1659 & .00077 & .00077 & 215 & 215 & \\
\hline $\begin{array}{l}\text { Cowboy } 10 \\
4 \mathrm{MPa}^{2} \\
8 \mathrm{MPa}\end{array}$ & 4.572 & $1.082 \times 10^{9}$ & 0.90 & .3316 & .00155 & .00093 & 214 & 356 & $\sim 150$ \\
\hline $\begin{array}{l}\text { Cowboy } 12 \\
4 \mathrm{MPa} \\
8 \mathrm{MPa}\end{array}$ & 1.829 & $1.053 \times 10^{9}$ & 14 & .3227 & .00236 & $\begin{array}{l}.00924 \\
.00398\end{array}$ & 136.8 & $\begin{array}{l}34.9 \\
82.1\end{array}$ & -20 \\
\hline $\begin{array}{l}\text { Cowboy } 14 \\
4 \mathrm{MPa} \\
8 \mathrm{MPa}\end{array}$ & 1.829 & $2.157 \times 10^{9}$ & 29 & .6611 & .0535 & $\begin{array}{l}.0737 \\
.0156\end{array}$ & 12.4 & $\begin{array}{r}9.0 \\
42.4\end{array}$ & -10 \\
\hline 1 kt HE & 6.78 & $4.186 \times 10^{12}$ & & 1283 & & & & & \\
\hline $\begin{array}{l}1 \mathrm{kt} \text { Nucl. } \\
(5.33 \mathrm{MPa} 0\end{array}$ & 0.5 & $4.186 \times 10^{12}$ & & 612 & & & & & \\
\hline Salmon & 0.5 & $2.219 \times 10^{13}$ & & 2480 & & & & & \\
\hline $\begin{array}{c}\text { Steriing } \\
4 \mathrm{MPa} \\
8 \mathrm{MPa}\end{array}$ & 17 & $1.591 \times 10^{12}$ & & & 1.45 & $\begin{array}{l}2.74 \\
1.64\end{array}$ & $12:$ & $\begin{array}{r}65 \\
108\end{array}$ & -54 \\
\hline
\end{tabular}




\section{Calculation of Reverberating Pressure Sources}

Measurements by Murphey ${ }^{8}$ on cavity pressures in some of the cavity shots showed that the pressure history at the cavity wall was not a simple step in pressure, but included a spike followed by an oscillatory signal. In the soc calculations described in the preceding sections, the actual geometry of the energy sources had been simplified by the replacement of the explosive device and surrounding air by an equal mass of iron vapor filling the cavity uniformly. This idealized geometry produced only a step in pressure if the cavity wall did nor move outward appreciably. Ccnsequenty, in zider to reproduce in the $S O C$ calculations the reverberating pressure that was observed experimentally, it was necessary to simulate the actual source more closely.

For SOC input, the Pelletol explosive source for a particular cowboy shot was included as the actual weight of explosive multiplied by a factor of 1.1 to provide for the mass of the container and supports, and tiıis assembly was assigned a density of $1.0 \mathrm{~g} / \mathrm{cc}$. The cavities had been evacuated down to about one-twentieth of nurmal air pressure for each shot, so the density of the air in the SOC inpec was set at $6.25 \times 10^{-5} \mathrm{~g} / \mathrm{cc}$.

Finite difference calculations are not well suiced to problems where there are large density differences from one region in the problem to another, as Erom the pelletol to the air, and it is necessar; to use very smali time ster's per cycle to obtain reasonable results. Consequently, it was necessary to breal. each Cowboy cavicy problem into two parts, the first consisting of calculating the dynamics of a cavity surrounded by a rigid wall, and the second consisting of calculating the dynamics of the salt medium surrounding the cavity when subjected to the pressure history at the cavity wall as calculated in the first part. This procedure is valid where the outwart motion of the cavity wall is small, so that the benavior of the cavity gas is not much affected by replacing the air-salt interface with a rigid wall.

\section{Cowboy Shots--Reverberating Pressure Inpur}

Figure 44 compares the pressure at the wall of the $15-f$ t cavity for Cowboy shot 10, calculated with SOC, to the experimental results reported by Herbst et ai." These results are quite similar, indicating that the soc calculations did give realistic results. The details of the decay of the reverberations differ, however. In the experimental situation, the decay was 


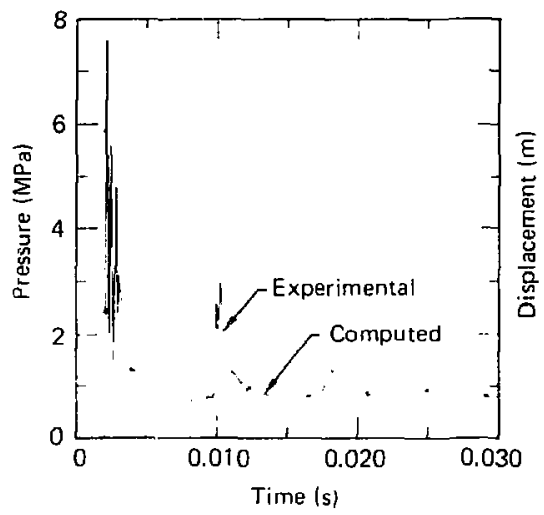

FIG. 44. Comparison of compuced and measured pressure at the wall of the cavity in Cowboy shot 10 .

frobably cassed by viscosity of the gases and by departures from spherical symmetry, which break up the reverberating wave. In the compuced case, the decay was probably caused primarily by the arcificial viscosity included to statilize the calculations, and dy the finite zone size.

The peak trotions as measured in the salt medium near che cavicy were those resultirg from the first pressure soike, so that the peak tisplacement did not depend on the decay rate of the reverberations. On the orher hand, in spectral studies the decay rate should havi a direct effect.

The Soc calculations do not give reliable results on the height of the pressure spike because this spike ordin.rily involves a shock front narrower in spatial extent than the zone size. The calculated values of the momentum impulse, which are related to the time integrals of the pressure spikes, are basic to the SuC calculations and should be more reliable. When the inomentum pulse accurs in a time that is short compared with the characteristic time of the cavity, then the motion of the medium depeuds primarily on this momentum pulse rather than on the height of the gressure peak, assuming that the mediutn remains elastic. If the positive phase is followed in a very short time interval by a negative phase, then some cancellation occurs, and tre seismic coupling is decreased. 


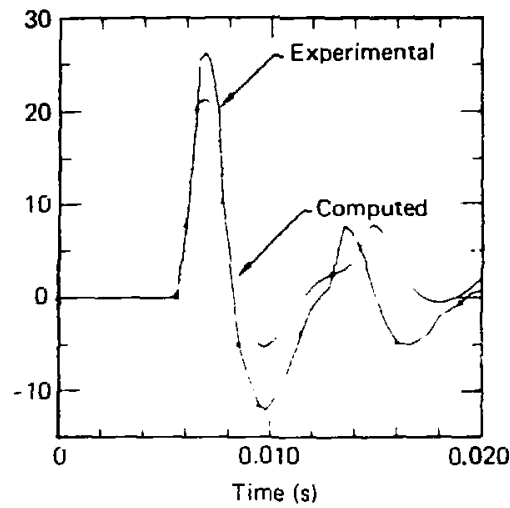

FIG. 45. Comparison of computed displacement at a distance of $80 \mathrm{ft}$ for Cowboy shot 10 with experimental results.

Figure is compares the displacement calculated using FFT for a poinc 30 tit from the center of the 15-ft cavity, possessing this input pressure histury, with the experimental curve. These curve; agree qualitarively. further verifying the suc calculations. The same displacement curves were uDtained for salt shear strengths of \& MFa and $8 \mathrm{MPa}$, indicating tnat unly elastic inceractions occurted in the calculations.

rigure it shows the calculated pressures at the wall of the is-ft cavity for Cowboy shots $6, B$, and 10 , and Fig. 47 shows the pressures at the wall of the b-it cavity for shots 12 and 14 . In these calculations the reveruerations appear to die away faster for the larger shots,

In shots 6 and 8 the peak pressures calculated for the cavities andicated tnat the resulting stresses in the surrounding salt should have been too low to lead to iallure. For chese problems the FFT procedures, which are valid for elastic motion, were used to calculate ile dynamic behavior of the salt. For Cowboy shots 10,12 and 14 , the cavity pressures were considerably higher, and for these shots the SOC code was used for the salt calculations, where inelastic behavior could be provided for, although tne mutions in shot la appeared to be elastic in che calculatinns. 


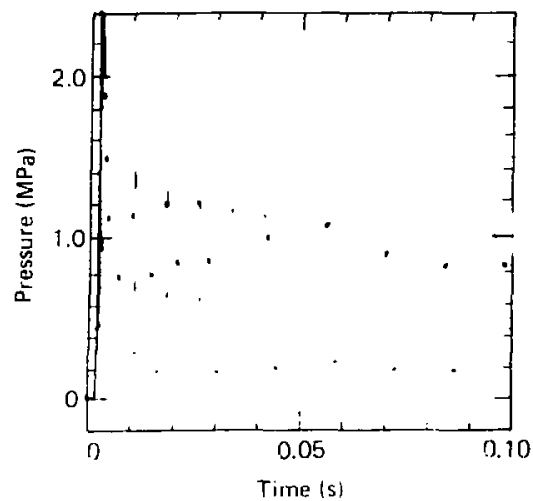

ili. ar. sinuced pressure nistorles at [i.t Elvity wall tor Cowboy shots

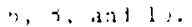

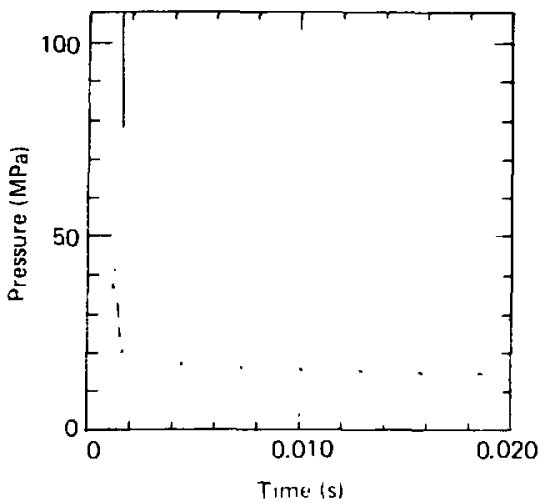

$F[1,+7$. Compised prassire nisturbes at the cavity wall tir iuw jy shots 12 and 14.

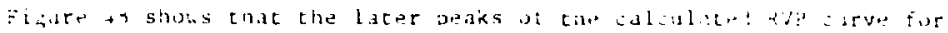

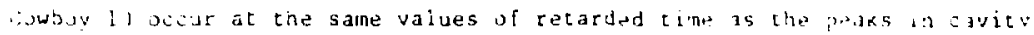

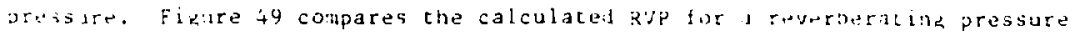

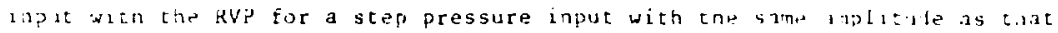

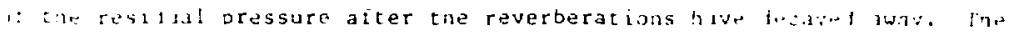

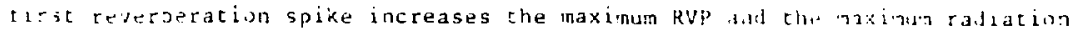

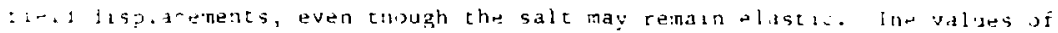

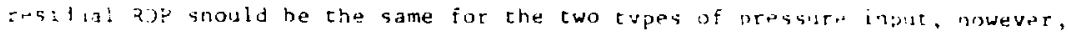

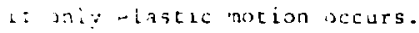

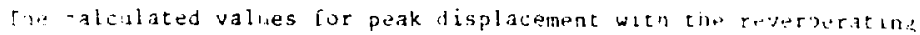

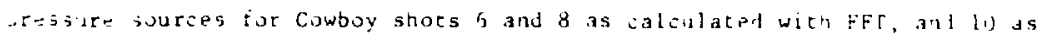

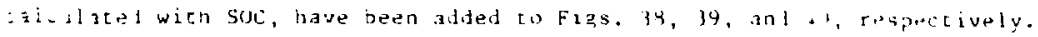

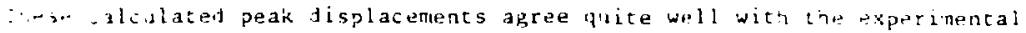
$\therefore \ldots$

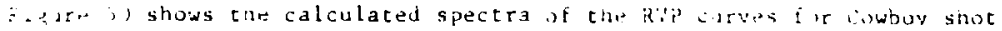
. : st., pressure input, for reverberating pressure insit, dial for a tamped

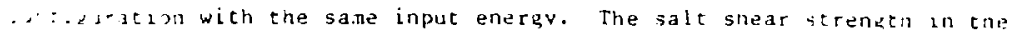
:a; : aiculation was set at 8 MPa, while for the cavity calculations it was

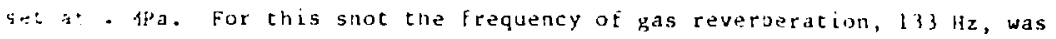
apprexintioly equal to the corner frequency of the cavity is ft in radius. an 


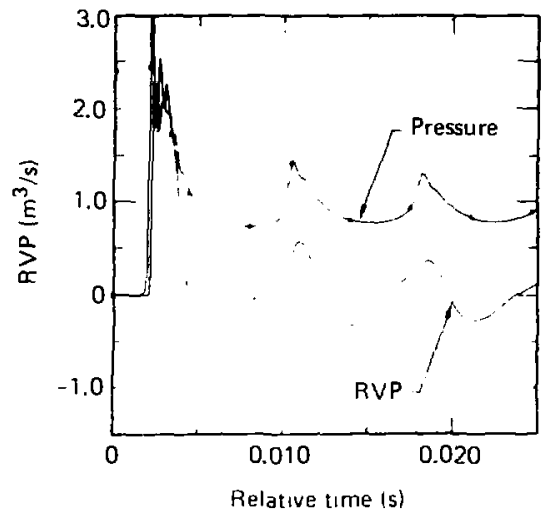

FIG. th. Relation at compuced rvp curve t) the inpul cavity presaure reverserationi tor Cowbuy 1 J.

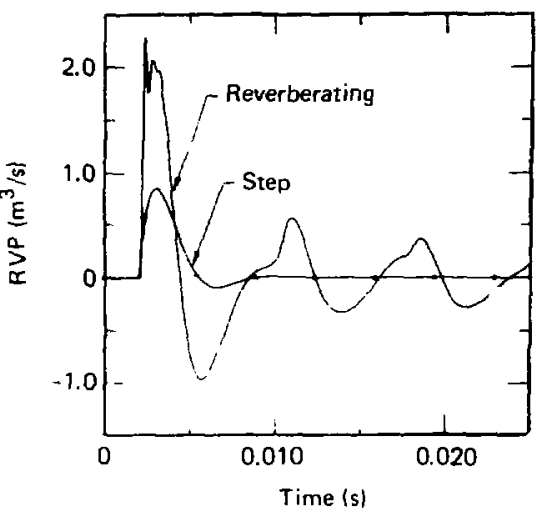

FIG. 49. Computed RVP histories for Cowboy snot 1) with step and with reverberating aavity pressure inputs.

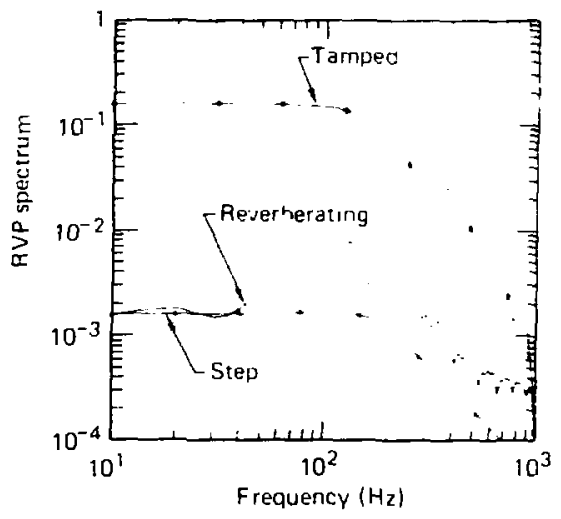

FIi. 50. Computed RVP spectra for Cowboy shot 10 with step and with reverberating pressure inputs at the savity wall, and in tamped geometry. 


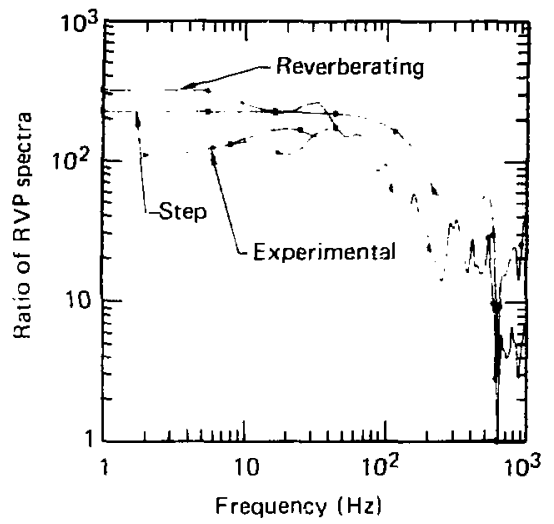

FIG. 51. Computed decoupling ratios for Cowboy shat 10 with step and with reverberating pressure inputs at the cavity wall.

interestar sucome is that the raverberating input pressure zave a resulting value iur risilial RDP lower thar that with step pressure, even though the values af late pressure in the cavity were the same, 0.90 4Pa. This result ay indicate that the inelastic motion resulting from the pressure spike relievel sine of the stresses associaced with the overburden pressure. Fizure il compires the resulting decoupling curves with experimental data. The calculated values of decoupling are higher than the experinental values rpourted 7 Herost et al. 9

Fizures i2 to 55 show some results of the calculations on sinot 12 . Figure 52 compares the RVP signals with step and with reverberating input pressures. The large amount of inelastic motion resulting from the reverberacing signal in the weakened salt (4-MPa shear strength) increased the anplicute of the RVP curve greatly. Figure 53 compares the RVP curves resulting from elastic motion only, in salt with an 8-Mpa shear strength and in salt with a 4-MPa shear strengtil. The inelastic yielding of the salt increased both the amplitude and the width of the first positive excursion of the KVP signal. Figure 54 compares the RVP signals from a camped explasion, from a reverberating pressure input in the cavity, and from a step change in pressure in the cavicy. For the camped shot the shear strength of tne salt 


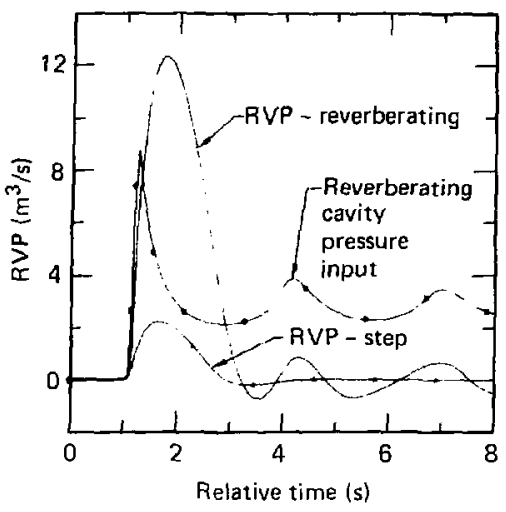

FIG. 52. Computed RVP histories for Cowboy shot 12 with step and with reverberating cavity pressure inputs. Also shown is the reverberating cavity pressure input, for timing comparison.

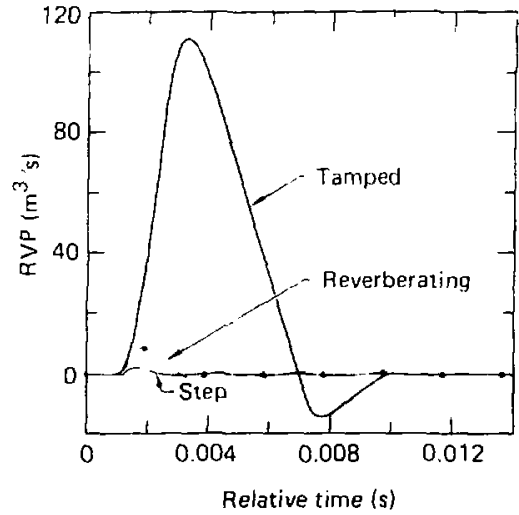

FIG. 54. Computed RVP + istories for Cowboy shot 12 with step and with reverberating pressure inputs at the cavity waI, and in tamped geometry.

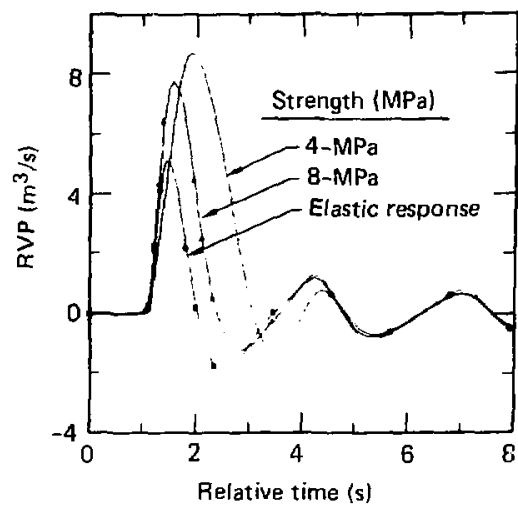

F[G. 53. RVP histories for Cowboy shot 12 as computed with FFT tc show the elastic response, and as computed with SoC to show the effects of the inelastic deformation, with reverberating cavity pressure input.

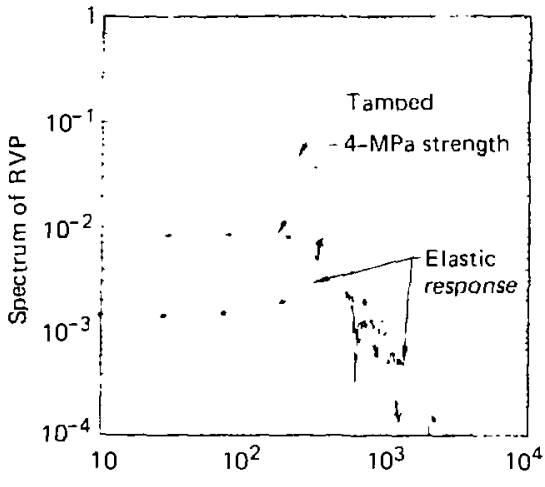

Frequency $\{\mathrm{Hz}\}$

FJG. 55. Computed RVP spectra for Cowboy shot 12 with reverberating pressure inputs at the cavity wall, and in tamped geometry. Also snown is the curve for reverberating cavity pressure input with elastis rasponse of the medium. 
was $8 \mathrm{MPa}$, while for the reverberating and step pressure cavity shots it was $4 \mathrm{MPa}$. Figure 55 shows the computed RVP spectra for a tamped shot, a cavity shot with a 4-MPa shear strength, and one with infinite shear strength (elastic solution). Figure 56 compares the resulting decoupling ratio curves with the experimental data.

Similar comparisons are made in Figs. 57 to 61 on Cowboy shot 14 . Figure 57 compares the RVP signals for $4-\mathrm{MPa}$ and 8-MPa salt shear strengths. In the calculation with the 4-MPa shear strength, even the second reverberating pressure peak contributes appreciably to the RVP signal. The curves of Fig. 58 show the large increase in RVP signal amplitude and duration caused by the inelastic motion. In Fig. 59 a graphic comparison is made among the RVP signals for a tamped shot and for cavity shots with reverberating and with step pressure input. Figure 60 shows the RVP spectra for a tamped shot and for cavity shots with 4- and 8-MPa salt shear strengths, and Fig. 61 shows the resulting decoupling curves. The decoupling ratio curve for the calculation with 4-MPa shear strength agrees moderately well with the experimental data, although the experinental results show a rise in decoupling with frequency. The computed curve shows only a slight increase at the corner frequency.

Table 7 lists the calculated values of residual RD, equal to the net positive area under the Rvp curves.

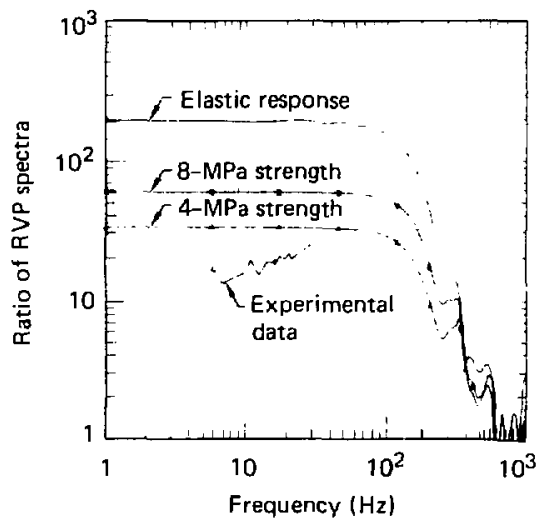

F[G. 56. Computed decoupling ratios for Cowboy shot 12 with reverberating pressure inputs at the cavity wall. Also shown arc the experimental data as given by Herbst et al.9 


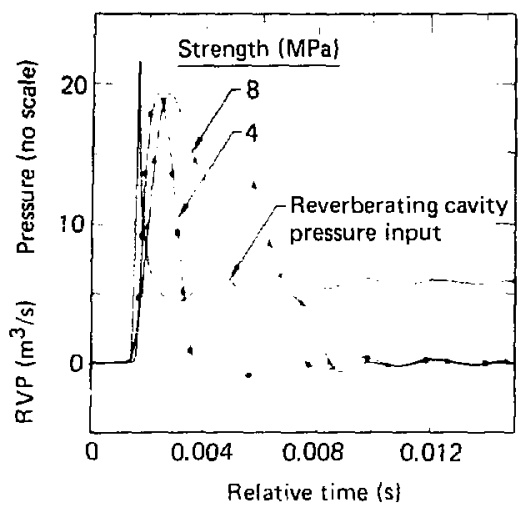

FIG. 57. Computed RVP histories Eor Cowboy shot 14 with reverberating cavity pressure inputs. Also shown is the reverberating cavity pressure input for timing comparison.

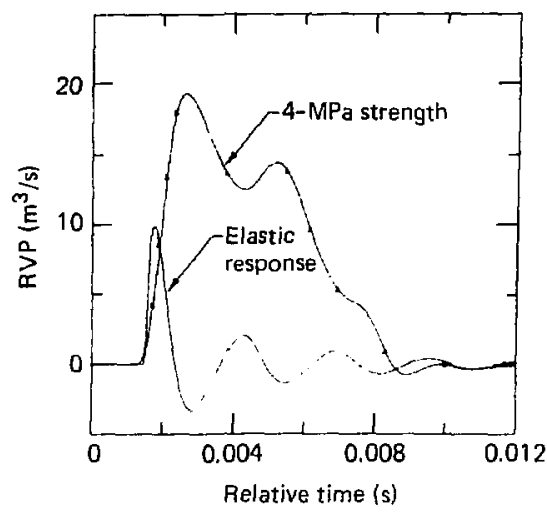

FIG. S8. RVP histories for Cowboy shot 14 as computed with FFT to show the elastic response, and as computed with SOC to show the effects of the inelastic deformation, both with reverberating cavity pressure input.

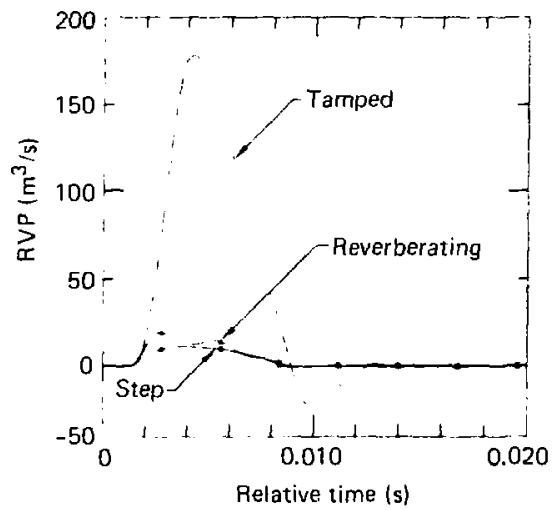

FIG. 59. Computed RVP histories for Cowboy shot 14 with step and with reverberating pressure input at the cavity wall, and in tamped geometry. 


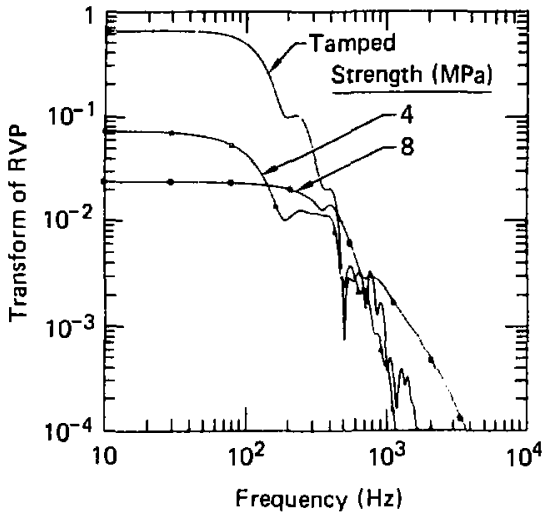

FIG. 60. Computed RVP spectra Eor Cuwboy shot 14 with reverberating pressure input at the cavity wall, and in tamped geometry.

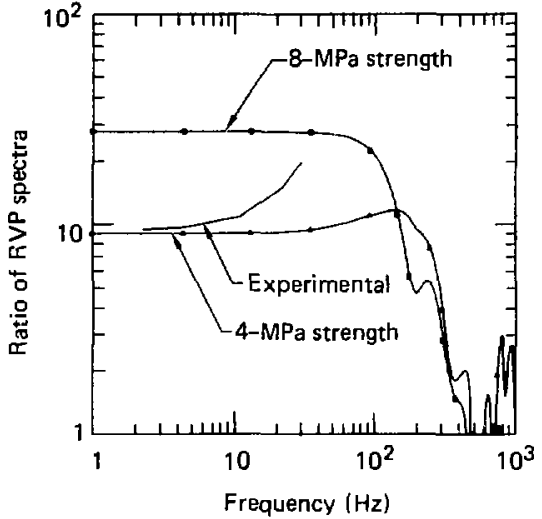

FIG, 61. Computed decoupling ratios Eor Cowboy shot 14 with reverberating pressure inputs at the cavity wall. Also shown are the experimental data given by Herbst et al. 9

These calculations on the cavity shots with the lowered shear strength of the salt are oversimplified, in that the strength of the salt was decreased to $4 \mathrm{MPa}$ at all radial distances. Actually, the salt nearest the cavity would ie degraded in strength the most, and the salt should be less affected with increasing radial distance. When the cavity $6 \mathrm{ft}$ in radius in a salt medium of 4-MPa shear strength, under the 5.33-MPa overburden pressure, was relaxed, inelastic motion took place out to a radial distance of $6.5 \mathrm{~m}$. In the subsequent dynamic calculation for Cowboy shot 14 , the outer extent of inelastic motion was about $9 \mathrm{~m}$, although at this distance the salt should have retained its original strength of $8 \mathrm{MPa}$. The exact variation of shear strength of this impure medium with inelastic strain is not known, so that an accurate curve of shear strength vs radial distance is not available.

\section{STERLING}

\section{Step Pressure Input}

In Project Dribble the Salmon event consisted of a 5.3-kt nuclear explosion in the salt medium. Extensive ground motion measurements were made 
within $744 \mathrm{~m}$, and also at regional and teleseismic distances. About 2 yr later the 0.38-kt sterling shot was fired in the cavity created by the Salmon explosion, and ground motion measurements were made again, in part with the same instrumentation that had been used in Salmon. The resulting data on Sterling were much lower in amplitude and of lower quality than the data from Salmon. The Salmon event created a cavity with radius of $22.3 \mathrm{~m}$, as estimated by perret. This cavity contracted in size, and 6 mo after salmon the radius was measured at $17.4 \mathrm{~m}$. The cavity volume as measured by Rawson et al. 21 was $19,600 \mathrm{~m}^{3}$, giving a radius of $16.7 \mathrm{~m}$. A value of $17.0 \mathrm{~m}$ was used in Sterling calculations here.

The coupling of Sterling was measured by Sisemore, Rogers, and Perret, ${ }^{12}$ and some of their results are presented here for comparison with the results from SOC. Figure 62 shows their data on maximum values of displacement. These points have a large scatter, and do not define a straight line well.

In the SOC calculations, the shear strength was again set at $8 \mathrm{MPa}$, and the input overburden pressure was 18.1 MPa. Preliminary relaxation calculations were cairied through on a mined cavity, and the resulting stresses were very similar to those shown in Figs. 31 and 32 . The sterling input energy of $9.38 \mathrm{kt}$ gave a calculated step in pressure of $17 \mathrm{MPa}$ in the 17-m radius cavity. Figure 62 also shows the peak displacements calculated with SOC, with fair agreement with the sattered experimental points. This agreement is in contrast to the results with the step pressure inputs for all the sowboy shots.

\section{Reverberat ing Pressure Input}

In the Sterling event, most of the mass present inside the cavity was that of the air, in contrast to the Cowboy shots, where nost of the mass was in the form of chemical explosive. The Sterling working point was $754.1 \mathrm{~m}$ below sea level and the temperature of the air in the salmon cavity was about $66^{\circ} \mathrm{C}$, so that the air density was about $1.14 \mathrm{~kg} / \mathrm{m}^{3}$. The total mass of the air in the cavity of radius 17 m was therefore some $23,650 \mathrm{~kg}$, which is much greater than that of the nuclear explosive assembly, consisting of a few hundred kilograms.

Since the temperatures involved might have been high enough to involve energy transport by radiation flow, a one-dimensional code that included 


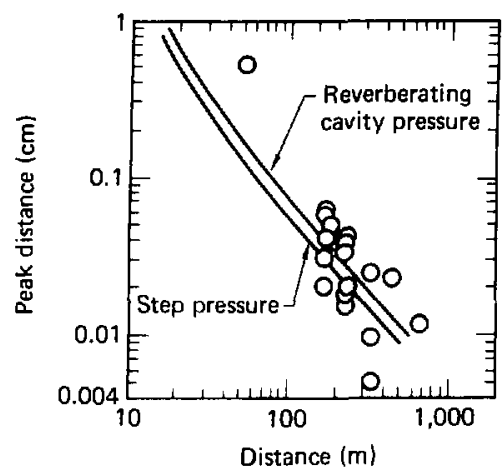

FIG. 62. Comparison of computed peak displacements with experimental data for the Sterling event. Energy input was $0.38 \mathrm{kt}$ and cavity radius was approximately $17.0 \mathrm{~m}$.

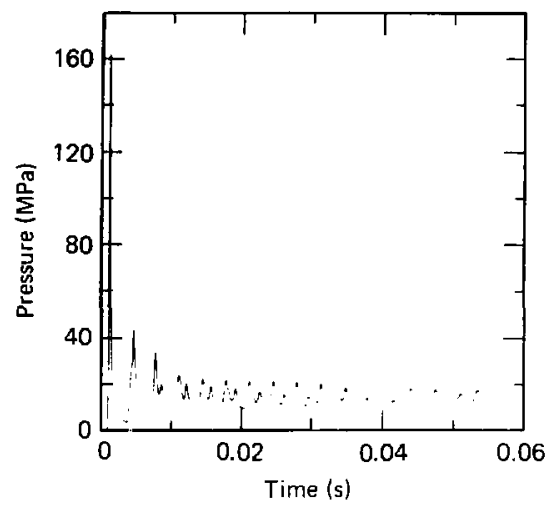

FIG. 63. Computed pressure history at the cavity wall for the sterling event.

radiation flow was used in place of SOC for the preliminary calculation of the reverberating pressure at the cavity wall. This reverberating pressure history, shown in Fig. 63, was then used for the subsequent sid calculations.

since the cavity in which the sterling shot was fired had been explosively formed by the salmon detonation, a large amount of iafiastic flow of the salt surrounding the cavity had occurred. Some additional inelastic flow occurred as the stresses relaxed to the condition of zero pressure inside the cavity. The post-shot velocity logs in Fig. 64 as presented iy perret ${ }^{18}$ show that the compressive and the shear wave velocities were appriciably decreased in the vicinity of the Salmon explosion. This indicates that the elasticity relations had been changed, and introduces the probabilitv that the shear strength had also been altered by Salmon and by the subsequent stress relaxation. In the calculations on sterling, the salt shear strength was set at $4 M^{\mathrm{T}} \mathrm{T}$ as in the cavity shots in cowboy. Additional calculations were made at a hear strength of 8 MPa for comparison with the 4-MPa calcilations.

Figure 65 show 3 the resulting RVP curve calculated with the SOC code with the reverberating input pressure, along with the pressure signal for an indication of the time relations between the features of the two curves. For this event, the reverberation frequency was much higher than the 


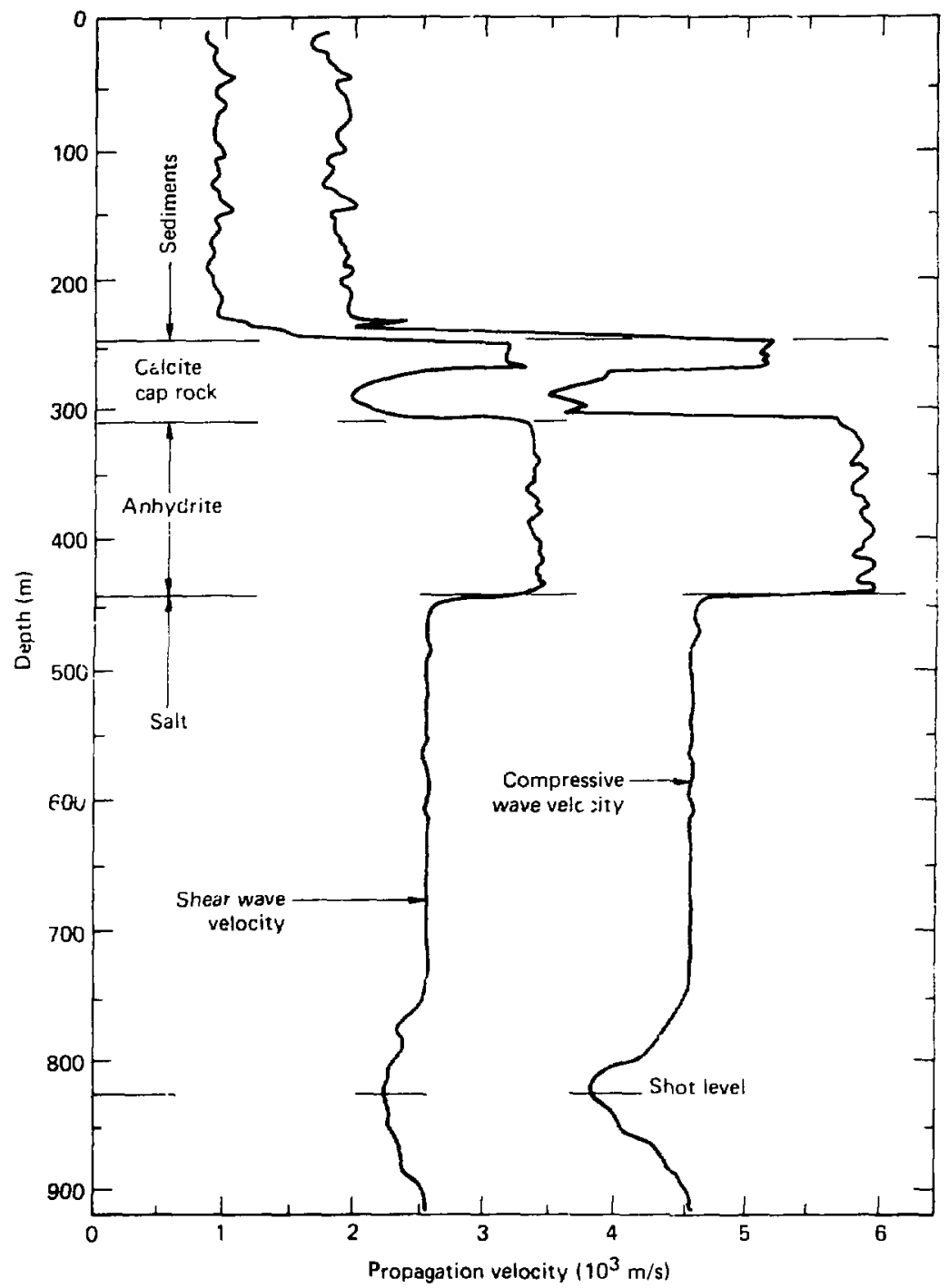

FIC. 64. Post-shot velocity $10 \mathrm{~g}$ in hole offset about $30 \mathrm{~m}$ from the salmon shot hole. (Adapted from Reference 18.) 


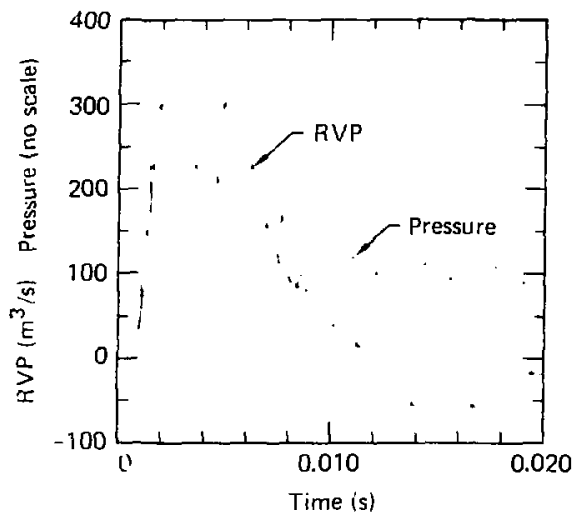

Flis. 65. Relation of computed RVP curve to the input cavicy pressure reverberations for sterling.

characteristic frequency of the cavity because of the high energy-to-mass ratio insude the cavity.

The calculated peak displacements with the reverberating pressure input have bepn added to $F i g$. 62 , where it is noted that this curve $i$, not much higher than that obtained with the step pressure input to the SOC colculations. This same feature is noted in Figs. 65 and 57 , where the RVP curves for the step and the reverberating pressure inputs are compared. The RVP curve with the step input shows the characteristic time of the cavity, while the RVP curve with reverbera ion input also shows this response but with the effects of the high frequency reverberations superimposed. The RVP spectra a. * rouparei in Fig. 67. It is seen that the reverberating source leads to some higher frequency concrubutions, with an RV peak occur-ing well beyond the corner frequency, but that the zero-frenuency amplituda kaqual to residual RDP) is only slightly hi,her than that from the step sourct. jince the residual pressures were the same for the two sources, this incrase in $w(\infty)$ was calised by the greater amount of inelastic deformation that occurred with the reverberating pressure input.

Figure 68 compares the RVP spectra as calculated with soc of sterling, of Salmon, and of sterling and Salmon scaled to an energy of $1 \mathrm{kc}$. The Sterling 


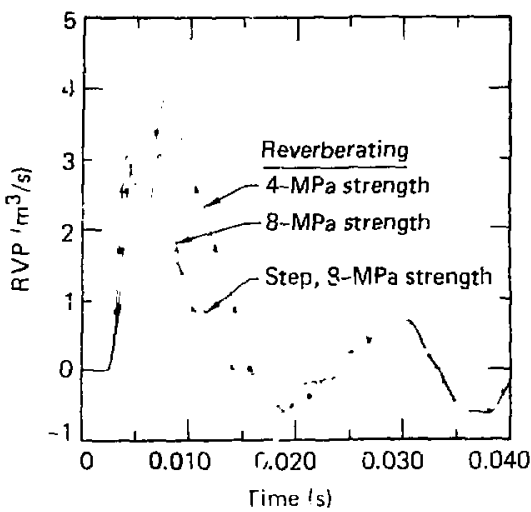

FIG. 66. Compuced RVP histories for sterling with step and with reverberating cavity pressure inputs.

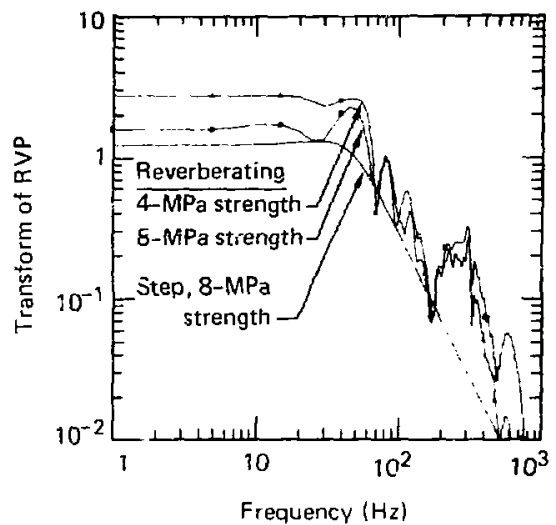

FIG. 67. Computed RVP spectra for Steriing with step and with reverberating cavity pressure inputs at the cavity wall.

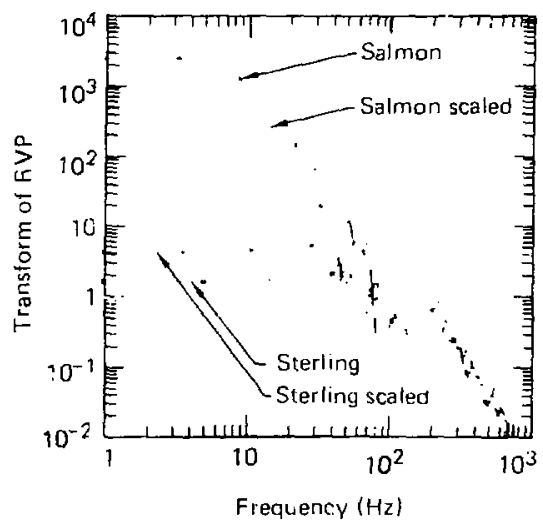

FrG. 38. Computed RVP spectra for

scerling, for salmon, and for

Sterling and Salmon scaled to $\mathrm{l} \mathrm{kt}$.

Reverberating input cavicy pressures

were used for the Sterling

calculations. 
6pectrum concains considerably more high frequency components in a relative sense, even when scaled to the same input on rgy.

The decoupling ratio was obcained as the ratio of the calculated RVP spectra for Salmon and Sterling converted to an energy level of $1 \mathrm{kt}$, and is shown in Fig. 69, along with the experimental curve presented by Murphy. 29 The agreement is good, although the calculated results do not show the increase in decoupling at about a $4-\mathrm{Hz}$ scaled frequency, and difter in the neighborhood of the corner frequency. Also shown is the decoupling curve obtained from soc calculations with a salt shear strength of 8 MPa. This curve lies appreciably above the experimental curve.

Table 7 lists the calculated and experimentally determined values of decoupling for Cowboy and for sterling, along with residual RDP.

\section{ANALYSIS OF CAVITY REVERBERATION}

Since the reverberating pressure sources in a cavity appear to have a significant effect on the earth motion, a study of the cavity dynamics was

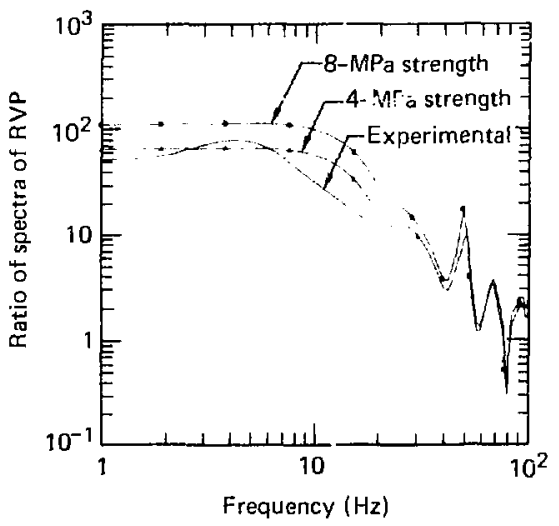

FIG. 69. Comparison of the computed and experimental decoupling curves for Sterling with respect to Salmon, scaled to l kt. Reverberating input cavity pressures wore used for the sterling calculations. 
undertaken. When an explosive is detonated in a cavicy, the detonation wave inoves outward through the explasive from its point of initiation. A shock wave is created in the air at the air-explosive interface, but because of the mass mismatch at the interface, only a small part of the energy is cartied by this shock wave. The remaining energy moves outward with the expending gas from the explosion, at a velocity lower than that of the shuck.

When the shock wave reaches the cavity wall, it is reflected because of the mass mismatch, and this reflected shock moves inward and meets the contact surface between the HE gas and the air. Although tne gas has become more rarefied at thc contact surface, the reflected shock can still be reflected outward again. These inceractions lead to a hashy pressure history following the first spike. Eventually the mass of HE gas nears the wall, and is slowed and reversed by tile pad of compressed air. This mass of gas moves back to the center, is reflected there, and the next reverberating cycle begins.

Figure 7$)$ shows the structure of the first pressure pulse in Cowboy shot 1.) as calculaced with SOL, along with the expericiental curve. This plot shows the rirst air shnck, the minor secondary shocks, and the broader pulse from the arrival of the $\mathrm{HF}$ pas. As seen in Fig. 63 , this oulse is followed by a trough, the pressure dropping to a value lower than the long-cerm stead, stare value.

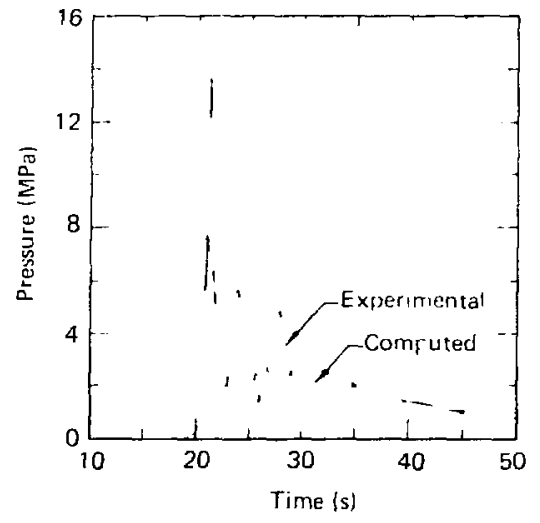

F1G. 70. Comparison of computed pressure history at the cavity wall with experimental data for cowboy shot 10. The time scale is expanded. 
The rise time of the first pressure pulse is very short because of the shock in the air. In the following pulses the waveform is still peaked because the moving gas mass is still concentrated. Eventually this localized motion diffuses down into the lowest mode of oscillation of the gas in the cavity, and the pressure history at the cavity wall becomes sinusoidal in form. The average pressure in the cavity is given by the relation

$$
P=(Y-1) E / V
$$

where $E$ is total energy and $V$ is volume. This energy inclides both the sutput energy frum the explosive and the initial internal energv in the air. The sonic velocity in the cavity gas is given by the relation

$$
\begin{aligned}
c^{2} & =\frac{r}{i} \\
& =\underline{P}(y-1)=:
\end{aligned}
$$

where $r$ is densily and $y$ is total mass in the cavity.

Ine 1 swest rasonant frequency in the acoustic approximation is stuwn in Append $x$ F in De

$$
t_{4}=\frac{4+ \pm 135 r g}{2 \pi a}
$$

The resonant period is the inverse of Eq. (13):

$$
\begin{aligned}
\Delta \mathrm{L}_{\mathrm{r}} & =\frac{2 \mathrm{a}}{4.4935 c 8} \\
& =\frac{2 \mathrm{a}}{4.4935}\left[\frac{\mathrm{M}}{\left.Y_{(Y}-1\right) E}\right]^{1 / 2}
\end{aligned}
$$

In the Cowboy shots the mass was mostly that of the pelletol gas, with $r$ equal to 1.25. In Sterling the mass was mostly that of the air, witn an assumed value for $Y$ of 1.2 . The periods obtained from the daca in lable 8 with the use of E7. (20) are listed in Table 9, along with the periods obtained from the SOC calculations. Also listed are the values from the Cowboy experimental data for the shots where this information could be 
TABLE 8. Masses and energies of the explosives and of the air in the Cowboy cavity explosions and sterling.

\begin{tabular}{|c|c|c|c|c|c|c|c|}
\hline \multirow[b]{2}{*}{ Shot } & \multicolumn{3}{|c|}{ Mass (hg) } & \multicolumn{3}{|c|}{ Energy (MJ) } & \multirow{2}{*}{$\begin{array}{c}E / M \text { ratio } \\
(M J / k g)\end{array}$} \\
\hline & Explosive & Air & Total & Explosive & Air & Total & \\
\hline \multicolumn{8}{|l|}{ Cowboy } \\
\hline Shot 6 & 99.8 & 25.0 & 124.8 & 226.8 & 10.0 & 236.8 & 1.897 \\
\hline Shot 8 & $23 R .2$ & 25.0 & 263.2 & 541.4 & 10.0 & 551.4 & 2.095 \\
\hline Shot 10 & $475 . n$ & 25.7 & 501.0 & 1081.8 & 10.0 & 1091.8 & 2.179 \\
\hline Shot 12 & 453.5 & 1.57 & 465.1 & 1053.5 & 0.63 & 1054.1 & 2.266 \\
\hline Shot 14 & 949.2 & 1.54 & 930.8 & 2157.3 & 0.61 & 2157.9 & 2.270 \\
\hline Sterling & small & 23,420 & 23,420 & $1.591 \times 10^{6}$ & 8200 & $1599 \times 10^{6}$ & 67.3 \\
\hline
\end{tabular}

TABLE 9. Calculated and observed periods in cavity explosions. Calculations were made witn Eq. (21) and with the soc computer code.

\begin{tabular}{|c|c|c|c|c|}
\hline \multirow[b]{2}{*}{ Snot } & \multicolumn{3}{|c|}{ Gas period (msec) } & \multirow{2}{*}{ Cavity period $=\underset{m s e c}{c}\left[\frac{\pi a}{\mu}[2 \mu]^{1 / 2}\right.$} \\
\hline & $\overrightarrow{E q} \cdot(2 I)$ & SOC & observed & \\
\hline \multicolumn{5}{|l|}{ Cowboy } \\
\hline Shot 0 & 8.3 & 8.0 & & 7.6 \\
\hline Shot 8 & 7.0 & 8.0 & 7.5 & 7.6 \\
\hline Shot 10 & 7.7 & 7.7 & 8 & 7.6 \\
\hline Shot 12 & 3.0 & 2.8 & 2.6 & 3.0 \\
\hline Shot 1 : & 3.0 & 2.7 & & 3.0 \\
\hline Sterling & 5.9 & 3.4 & & 28.3 \\
\hline
\end{tabular}

obtained from the records. The agreement among the values from these throe sources is quite good.

The periods obtained from the acoustic approximation method are only approximate because the oscillations were nut infinitesimal in amplitude, and because the final cavity gas was not homogeneous, but consigted of separate regions of gas from the explosive and of air. Although these regions equilibrate to the same pressure at the contact surface, they do not necessarily reach the same sonic velocity. One gas may end up at a higher 
tomperature and lower density than the other, giving a higher sonic velocity. The reverberating gas inuld then act as two coupled oscillators, with two "fundamental" modes of oscillation. Furthermore, a radial variation of temperature and density within each gas would be expected, further complicating the gas motions.

The behavior of the first pressure pulse depends upon additional nonlinear factors not provided for in the acoustic analysis, and this first pulse is the component of the pressure wave that determines peak displacements and velocities at free-field distances. This pulse has a sceep rise because of the increase in velocity with pressure, which is tne basis of the formation of a shock, leading to greater high frequency content. The precise shape and amplitude of this pulse lep ids up in dimensions, densities, material properties, and energies involved i- '.e cavity explosion, but it is noted that in the Cowboy and Sterling cavity shots, the width of this pulse is somewhat less than the half-periods of the acoustic solutions.

It is observed in Figs. $4 F, \cdots, 47$ that the shapes and frequencies of the pressure waves are nearly dent of the amount $o$ oxplusive used. Sincr. the acoustic resonant pei. derands upon the ratio $M / 5$, in $F .7$. (20), and since most of the $m$. In the rowboy cavity shots was present as mass of explosive, increasing the amourt of explosive did not change the M/F ratio appreciably. In Sterling, on the other hand, mos. of the mass was in the air in the cavity, and an increase in nuclear energy outs:t would raise the resonant frequen-y.

The seismic transfer function of the cavity in the elastic range is that of an overdamped oscillator. A crmmonly used reference frequency for the cavity itself along with the surrounding medium is given by the relation

$$
\mathrm{F}_{0}=\frac{c}{2 \pi \mathrm{a}}
$$

where $c$ is the compressional sonic velocity in the medium. This frequency is iadependent of the cavity gas, and depends only on the cavity radius and on the properties of the external medium, and is approximately the corner frequency of the spectrum of the radiated displacement signal when a step in pressure is applied inside the cavity.

This frequency $E_{D}$ is not the resonant frequency of the savity, however. From Eqs. $(A-2),(A-6)$, and $(A-7)$ in Appendix $A$, it is found that the fourier transform for the reduced velocity potential has the form 


$$
\hat{\phi}(f)=\hat{p} \frac{a c}{i} \frac{l}{-\frac{j}{\omega}\left(\frac{c}{a}\right)^{2}-\frac{c}{a}+j \omega\left(\frac{\lambda+2 u}{4 \nu}\right)}
$$

Resonance occurs when the imaginary quantitie in the transfer function on the right-hand side cancel each other, resulting in a maximum of the absolute value of the transfer function. This occurs at

$$
\omega_{T}=\frac{c}{a}\left(\frac{4 u}{\lambda+2 \mu}\right)^{1 / 2}
$$

or

$$
\mathrm{f}_{\mathrm{r}}=\frac{\mathrm{s}}{\pi \mathrm{a}}
$$

where $s$ is the shear wave velocity in the surrounding medium. Rodean 39,40 has investigated the relations among the various quantities involved in elastic wave radiation from spherical sources using the quantity $f_{r}$ as defined in Eq. (25) as a prinary parameter.

Figure 71 givas the dependence of the radiated RVP or displaceme- on the rati, $f_{g} / f_{0}$ for sine-wave excitati $i$, as checked with soc calrulations. The curve shows that the amplitude of the resulting sinusoidal RVp has a maximum wile.s the frequency $\mathrm{E}_{\mathrm{g}}$ is somewhat greater than $\mathrm{F}_{0}$, Eqs. (2L), (22), and (23) give the result

$$
\frac{E_{F}}{E_{0}}=\left(\frac{4 \mu}{i+2 u}\right)^{1 / 2}
$$

When $\lambda=\mu$, as is the case for the salt medium considered here, $\mathrm{f}_{\mathrm{r}} / \mathrm{f}_{0}$ $=1.1547$.

The response curve of Fig. 71 drops off with unity slope on the log plot at lower and higher frequencies. When the cavity pressure is impulsive rather than ginusoidal, the efficiency of seismic radiation for each frequency component in che pressure curve will follow the transfer function shown in Fig. 71 if the medium remains elastic.

The periods associated with the cavity frequencies $f_{r}$ have been added to Table 9 for comparison with the periods associated with the gas frequencies. It is seen that these periods were almost equal for each Cowboy 


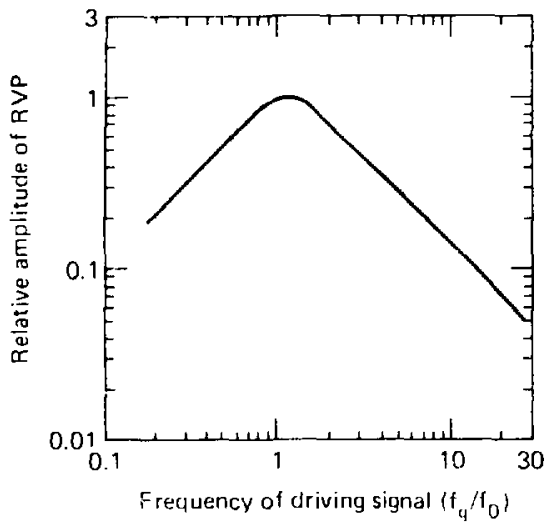

F1G. 71. Computed seismic coupling efficiency as a functiun of the $\mathrm{f}_{\mathrm{g}} / \mathrm{f}_{0}$ ratio (che ratio of the reverberation frequency to the cavity reference frequency).

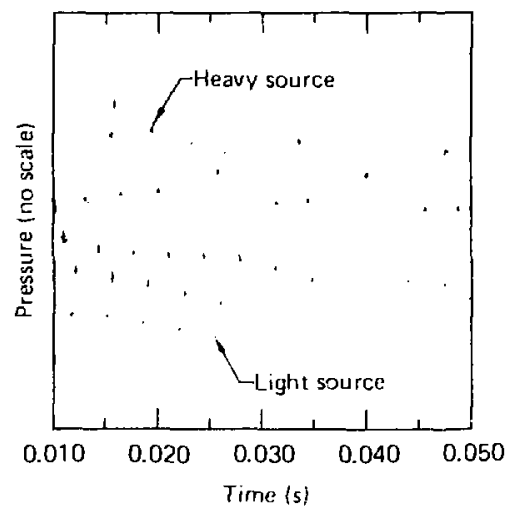

FIr; 72. Comparisins of computed pressure waveforms at the cavity wall in Sterling wit! a low-mass energy source and a high-nass source.

shot, but hat for sterling the gas frequency was much higher than the cavity frequency. In Figs. 50 and 67 the computed amplitude spectra at RVp for Cowboy shot 10 and Sterling, respectively, are presented for step and for reverberacing input pressures. For the Cowboy shots the excitation $p$ appear at the corner frequencies, while for stering the excilation pea far beyond the corner frequency. These spectra with the reverberating nut pressures art only qualitative because the correct decay rate of the waye train is not known, but they do indicate the signifirance of the $\mathrm{f}_{\mathrm{g}} / \mathrm{f}_{0}$ ratio.

Some of the inaccuracies in the calculated soc results could have been decreased by a more precise description of the geometry of the particular cavity shot. In the calculations it was assumed that the explosive and its coitainer would be vaporized by the explosion, but actually the container probably remained as a collection of solid particles as it moved toward the cavity wall. Other refinements, such as finer zoning, might be made in a more meticulous calculation.

The energy transport may take a different form in the nuclear explosions. If the mass of the device is low, then the early part of energy 
transfer is by radiation diffusion, without an appreciable transfer of inomentum. If the early fireball fills the carity, then the mass of air in the cavity is nearly in the form postulated for the step change of pressure, that of a uniforin gas at uniform pressure filling the cavity. On the other hand, if the mass-to-energy ratio of the nuclear device is high, then the energy transport will be through mass motion, and the reverberating pressure source will result. In an internediate case, the fireball will fill only part of the cavity, and the cavity gas will consist of the two regions of gas, one hot and rarefied and the other colder and denser, and the reverberation will be that of a cripled oscillator.

Figure 72 compares the pressure waveforms resulting from a ange in device mass. The lower curve gives the calculated pressure curve for a nuclear ievice with a relatively low mass, and the upper curve gives the pressure wavefor:a for a cevice whose mass is larger by a factor of 6.5 . The interaction sf tite coupled modes of oscillation is prominent in the lower curve, while for the upper curve the irregularities have almost disappeared.

Thr calculations with the heavier nuclear Jevice also gave a nuch higher initial spike in pressure, $242 \mathrm{MPa}$ as compared with $162 \mathrm{MPa}$ for the lighter device. The higher spike should lead to greater inelastic defornation in the surrounding medium, and to greater seismic coupling.

\section{DISCUSSION}

The purfose oîthis investigation was to explain decoupling in salt, both qualitatively and quantitatively, with the aid of computer calculations. This analysis was intended to $u$ ify all experimental data and observed phenomena inio a self-consistent pic, :e. In this work the degree of decoupling at distant seisnic stations was the ultimate quantity of interest, and this decoupling was determined primarily by calculation of the RDP and RVP curves.

Experimental measurements were made at close distances in free-field geonetry, and at large distances in geometries where the received signal has undergone many reflections and refractions. The free-field signals are ordinarily predoninantly high frequency signals and tho seismic signals are low frequency, with practically no overlap between the two. We made many efforts to determine values of residual RDP from the experimental data, since this quantity bears on the very low frequency component of seismic signals, 
but only efforts on the Salmon event were successful. The corresponding determination on Sterling was unguccessful, so we could not obtain the value of the very low frequency decoupling from the residual RDP values. The experimental difficulty was that for the free-field measurements, the transient displacements were much larger in amplitude than the residual displacements from shich residual RDP was to be determined, obscuring the residual signals. In addition, instrument drift was a pervasive problem in the low frequency measurements.

An alternate approach was the determination of the peak value of RVP, since it bore a direct relation to peak displacement. The practical disadvantages of this parameter as an indicating device are that at short distances the high irequencies are highly attenuated by the salt medium, and the elastic region for these frequencies is never reached. In the elastic region the pak displacements should atcenuate wi h distance as $1 / \mathrm{r}$, but in the experimental data the decay rate at the war distances was usually about $1 / \mathbf{r}^{1.5}$. Consequently, valid determinations of peak RVP could not be made from the experimental data.

The residsal mp does not suffer from this difficus:y. Residual PDP is an induction field; antity, and the displacement associated with residual RDP decays as $1 / \mathrm{r}^{2}$. The experimental data from Salmon do give this Jecay rate for residual displacement. Tiı siguir observed at the seismic stations is not "ero frequency and is a radiation field signal, but still the frequeacy spectrum is flat at very low frequencies up to frequencies approaching the $r \in f e r e n c e$ angular frequency $w_{0}=c / a$ of the cavity or of the inelasic sphere. Although the seismic signal is observed in the time domain, and the detecrability of this signal is determined by the poaks in the time domain, it is still necessary to convert to a spectrum in the frequency domain to eliminate the variations in signal peaks caused by multipath transmission. The single decoupling value as determined by a comparison of the peak signals is more satisfying and dirers, but for more consistent and complete results, the decoupling must be determined in the frequency domain.

Since the frequency spectra of the displacements at near and at geismic distances do not overlap, it is not obvious that free-field measurements have much importance in determining decoupling at seismic distances. In performing the calculations presented in this report, however, it became obvious that the inelastic motion associated with the high frequency spike made $=$ direct contribution to the zeromfequency signal. If the pressure spike did not 
drive the medium into the inelastic region, then the zero-frequency couplinB would remain unchanged from the case where the pressure source consisted of only low frequency componeats.

In these calculations the reverberating pressure signals were computed with only moderate accuracy because the shot geometries were not known precisely, hut the results from the calculations still agree fairly closeiy with the experinental data, both free-field and seismic.

Results from the calculations with the reverberating pressure source appear to make inapplicable all the results obtained with the step pressure input to the decoupling problems. On the other hand, the step in pressure represents an idealized input that can be applied to any combination of input energy and cavity radius, while the reverberating pressure sorices represent pressure histories from particular geometries. In the case of a nuclear explosion where the fireball fills the cavity, the pressure signal may contain almost no sjike, and may approach p function instead. The step pressure input may give results that may be rded as lower limits for the coupling.

The SOC calculations probably oversimplify actual rock tehavior. In SOC a precisely delimited failure curve is used, while in reality the failure may occur in a rather statistical manner. This simplification would cause the inelastic effects to disappear rather atruptly in tue calculated cu ve of coupling vs cavity radius, whereas in reaitity th a crange would be expected to be more gradual.

Another difficulty is that crack behavior, involving two- or three-dimensional behavior, cannot be analyzed adequately in a one-dimensional code. It may be that reasonable results are obtained by lowering the nominal shear strength of the medium in the computer calculations. To match the experimentally determined displacements in the Salmon calculitions it was necessary to use a value for shear strength of salt about one-fourth of the laboratory value.

It is often assumet that failure of the salt medium will occur when the transverse stress becomes tensile. With the use of Eqs. $(1 n)$ and (11), the radial and transverse stresses at the cavity wall can be expressed in terms of the cavity pressure $P_{c}$ and the overburden pressure $P_{0}$ :

$$
\begin{aligned}
& \sigma_{r}=-P_{c} \\
& \sigma_{t}=\left(P_{c}-3 P_{o}\right) / 2
\end{aligned}
$$


The radial stress is compressive for all values of cavity oressure, while the transverse stress at the cavity wall becomes tensile at

$$
P_{c}=3 P_{0}
$$

When tise shear strength is quite low, the salt around an umpressurized mined cavity will deform plastically until an equilibrium stress field is established where the maximum shear stress equals the failure shear stress $\sigma_{f}$. Upon application of a step in pressure in the cavity, this shear stress will decrease to zero, and will then increase in the alrernate direction until the failure level is again reached.

Although this reasoning is valid for perfect spherical symmetry, it may not be generally applicable in reality. In any departure from spherical geometry, such as in the case uf cracks extending out radially, there will be stress concentrations at the crack tips. Tensile failure cf the salt medium may then occur at cavity pressures that exceed overburden pressure to a much smaller extent. The limiting $\mathrm{r}$ ressure that could cause such failure wrild be $P_{c}=P_{o}$, which would certainly be valid for a fluid medium. For the salt medium, it would be valid where the pressurized gas flows into the cracks, transferring the region of high gas pressure out to larger radial distances and bypassing the restraining effects resulting from the rigidity of the salt med lum.

In the soc calculations, the salt is not permitted to go appreciably into tension, so that the tensile hoop stresses are relaxed automatically, giving the same effect as the transverse stress relaxation due to the radial cracks. There is no gas flow into the cracks in SOC though, so that in these computations there would be zero pressure inside the cracks.

Figure 28 shows the calculated variation of final cavity pressure with initial cavity radius, for an energy input of $1 \mathrm{kt}$. It is interesting and significant that for a partially decoupled shot, the calculated residual gas pressure remains high, with a peak of about $65 \mathrm{MPa}$ for the case shown, where shear strength is $8 \mathrm{MPa}$ and overburden p: essure $18.1 \mathrm{MPa}$. It is noted that in this case, the cavity pressure was more than three times cverburden pressure, contrary to Eq. (28). This simply means that inelastic deformation must have occurred to accommodate this additional pressure. In principle, this cavity pressure could have been arbitrarily high, with a corresponding increase in the radius of inelagtic deformation. 
This analysis applies only if the gas does not flow into the cracks, however. If the gas should flow into the cracks, hydrofracturing would ociur and would continue until the cavity gas pressure would be lowered almost to overourden prissure. The rate of hydrofracturing would depend upon the $v i s c o s i t y$ of the gas and the openness of the cracks.

The second observation to be made from Fig. 28 is that the residual ga: pressure for the tamped snots is much lower than for the partially decoupled shots, and at $9 \mathrm{MPa}$, is lower than overburden pressure. For this low gas pressure, hydrofracturing will not occur. The conciusion is that the phenomenon of hydrofracturing will occur only for the partially decoupled events, and the variation of coupling with overburden pressu" c whl differ for the tamped and fur the particlly decoupled events.

ln post-shot excavations around the cavity with radius of $6 \mathrm{ft}$, in which Liswoy snuts 12 ind 14 hal been ired, Short ${ }^{4 l}$ observed many carbon-filled cracks that extended out at least one foot into the salt tncdium, and several fractures that axtended to depths of 7 to $9 \mathrm{ft}$. Although these results do not provi: that the couplina was increased by gas flow into the cracks, they do sukgest thas possibi Li\%

\section{ACKNOWLEDGMENTS}

rhese analyses were proposed by perer Youlthrop, and advice dias aiven by Kobert Terhune as to the fitting of the experimental Salmon waveforms. Charles snell of LASL and Landon Bruce and Don Burton of LLivL devised the procedures necessary to relax the stresses around the cavities. Many of the actual machine calculations were carried ut by the cheerful and conscientious efforts of Mirian Lohmann.

A draft of this report was reviewed and cunstructive criticisms were given by Don Larson, Pete Moulthrop, Bob Terhune, Howard Rodean, and Jim itannon.

'The wurk discussed in this report was spons: :ed by the Advanced Research projects Afency and performed under the auspices of the U.S. Department of Energy. 


\section{REFERENCES}

1. A.L. Latter, E.A. Martinelli, and E. Teller, "Seismic Scaling Law for Underground Explosions," The Physics of Fluids 2(3) (1959).

2. A.l. Latter, R.E. Lelevier, E.A. Martinelli, and W.G. McMillan, "A Method of Concealing Underground Nuclear Explosions," J. Geophys. Res. 66(3) (1961).

3. A.L. Latter. F.A. Martinelli, J. Mathews, and W.G. McMillan, "The Effect of Plasticity on Decoupling of Underground Explosions," J. Geophys. Res. 66 (9) (196L).

4. N.A. Haskell, "A Static Theory of the Seismic Coupling of a contained Underground Explosion," J. Geophys. Res. 66 (9) (1961).

5. H.W. Lewis and S.B. Treituan, 'Seismic Signals Erom Nuclear Explosions in Overdriven Cavities," J. Geophys. Res. $71(8)$ (1966).

h. H.C. Rodean, Cavicy Decnupling of Nuclear Explosions, Lawrence livernore National Laboratory, 1.ivermore, Calif, JUCRL-51097 (1971).

T. H.C. Rodean, "An Energy Approach to seismic Coupling Analysis," J. Stophys. Res. $7(17)(1972)$.

8. R. F. Murphey, "Particle Motions Near Fxplosions in yalite," I. Geophys. Kes. $65(3)(1961)$.

9. R.F. Herbst, r..C. Werth, and D.L. Springer, "Use of large cavities to Reduce Seismic Waves from Inderground Explosions," J. Geophys. Res, 6h(3) (1961).

10. G. Werth and P. Randolph, "The Salmon scient ific Fxperimenr," J. Geophys. Ras. $71(14)(1966)$.

11. D. Epringer, M. Denny, J. :ealy, and $W$. Mickey, "The Sterling Fxperiment: Decoupling of Seismic Waves by a Shot-Generated Cavity," I. Geophys. Res. $73(18)(1968)$.

12. C.J. Sisemore, L.A. Regers, and W.R. Perret, "Project sterling: Subsurface Phenomenology Measurements Near a Decoupled Nuclear Event," J. Geophys. Res, 74(17) (1969).

13. H.L. Brode, Nuclear Explosions in Cavities, Rand Lorporation, Santa Monica, Calif., RM 3727 (1965).

14. L.A. Rogers, "Free-Field Motion Near a Nuclear Explosion in Salt: Project Salmon," J. Geophys. Res. $71(14)$ (1966).

15. D. W. Patterson, "Nucleor Decoupling, Full and Partial," j. Geophys. Res. 11(14) (1966). 
15. C. Godfrey, D. Maxwell, F. Finlayson, E.T. Moore, V. Davis, H. ifuises, R. Kant, G. Steel, and C. Mutobe, A Theoretical and Experimental Study of Detonations in Connection with Decoupling, Physics International Company, San Leandro, Calif., DASA 2505, DASA 0l-68-C-0147 (1969).

17. R.W. Terhunc, C.M. Silell, and H.C. Rodean, Enhanced Coupling and Decoupling of Ilnderground Nuclear Explosions, Lawrence Livermore National Laboratory, Livermore, CaliE, IICRL-52876 (2979).

18. W.R. Perret, Free-Field Particle Motion from a Nuclear Explosion in Salt Part l. Sandia i,aboratory, Albuquerque, N. Mek., VuF-3012 (1968).

19. I.F. Schat 2, Soc73, A One-Dimensional Wave Propagation Code for Rock Media, Lawrence Livermore National Laboratory, Livermore, Calif., IICRL-51589 (1974).

2n. C.M. Snell, "ser's Manual for Soc74, Lawrence Livermore National laboracory, Tivermore, Calif., RKCD 76-12 (1976).

? I. T. Rawson, R. Randolph, r. Buardman, and v. Theeler, "Post-Explosion Envirnmenc Resulting [rom the Salmon Event," J. Geophys. Res. $71(14)$ (1956).

22. 4.F. Avery and M.L. Rednond, Tridxial Compression Tests of Salc Rock Cores for the Inited States Atomic Energy Commission, Project Dribble, "1.S. Bureas of Reclamation, Denver, Coio., C-1043 (AEC TID-2 1592) (1962).

23. H.C. Heard, A.E. Abey, B.P. Bonner, and A. Duba, Stress-Strain Behavior of Polvcrystalline NaCl to ,2 GPa, Lawrence Livermore Nationa! Laboracurv, livermore, Calif., IICRI_-5:743 (2975).

24. I. Schatz, A. Kusubov, J. Hearst, A. Abey, C. Snell, and L. Thigpen, Rock Yechanic Project Propress and Resules: Rock Fracture and pore Coliapse, l.awrence Livermore vational Laboratory, Livermore, Calif., UCID-L752? (1977).

25. T. R. Butkovich, A Technique for Generating Pressure-Volume Relationships and Failure Fivelopes for Rocks, Lawrence Livermore National Laboratory, Livermore, Calif., JCRL-5144i (1973).

25. C. M. Snel1, User Instructions for the f-Ml! Generator Code, Lawrence Livermore National Laboratory, Livermore, Calif., RKCD 78-5 (1978).

:7. W. M. Wells, "Calculation of Shrinkage of the Salmon Cavity," J. Ceophys. Res. $74(10),(1969)$.

28. W. R. Perret, "Seismic Source Energies of Four Explosions in a Salt Dome," J. Geophys. Kes. $78(32)(1973)$. 
29. J.R. Murphy, "Discussion of Paper by D. Springer, M. Denny, J. Healy, and W. Mickey, "The Sterling Experiment: Decoupling of Seismic Waves by a Shot-Generated Cavity," J. Geophys. Res. 74(27) (1969).

30. R.A. Mueller and J.R. Murphy, "Seismic Characteristics of Underground Nuclear Detonations, Part I. Seirmic Spectrum Scaling," Bull. Seismol. Soc. Amer. 61 (6), 1675-1692 (1971).

31. J.R. Murphy and R.A. Mueller, "Seismic Characteristics of Underground Nuclear Detonations, Part II. Elagtic Energy and Magnitude Determinations," Bu11. Seismol. Soc. Amer. 61(6), 1693-1704 (1971).

32. D.B. Larson, "Spherical Wave Propagation in Elastic Media and Its Application to Energy Coupling for ramped and Decoupled Explosions," Lawrence Livermore National raboratory, Livermore, Calif., UCRL-52655 (1979).

33. J.A. Sharpe, "The Production of Elagtic Waves by Explosion Prassures. I. Theory and Empirical Field Observations," Geophysics $7(1-4)(1942)$.

34. F.G. Blake, JI., "Spherical Wave Propagation in Solíd Media," J. Acoust. Soc. Am. $24(2)(1952)$.

35. W.S. Derby, M.E. Hunnel, E.C. Lee, and R.D. Neifert, SOCKITTOME, An Interactive Data Processing Code, Lawrence Livermore National Laboratory, Livermore, Calif., UCID-17733 (1977).

36. J.H. Healy, Chi-Yu King, and M.E. O'Neill, "Source Parameters of the Salmon and Sterling Nuclear Explosions from Seismic Measurements," J. Geophys. Res. 76 $(14)(1971)$.

37. J. Trulio, Applied Theory, lnc., Los Angeles, Calif., letter to Carl Romney: "Factors obcained for full decoupling in salt by combining (a) measured salmon data with (b) calculations of salt motion due to bursts in large mined cavities" (April 3, 1979).

38. E. Lee, M. Finger, and $W$. Collins, JWL Equation of State Coefficient for High Explogives, Lawrence Livermore National Labo:atory, Livermore, Calif., UCID-16190(1973).

39. H. Rodean, Nuclear-Explosiou Seismology, AEC Critical Review Series, U.5. Atomic Energy Commission, Division of Technical Information, TID-25572 (1971).

40. H.C. Rodean, Elastic-wave radiation from spherical sources, Lawrence Livermore National Laboratory, Livermore, Calif., UCRL-52867 (1979). 
41. N.M. Short, Failure of Rock Salt by Explosive Loading at the Winnfield Salt Mine, Lawrence Livermore Nacional Laboratory, Livermore, Calif., UCRL-6054 (1963).

$\mathrm{JCC} / \mathrm{jmp}$ 


\section{APPENDIX A: RDP AND DISPLACEMENT RELATIONS}

It has been found that the chree-dimensional equations of elasticity where variations occur only in the $r$ coordinate can be solved in terms of a reduced displacement potential $\psi$. This function represents a wave moving outward, dependent only on the retarded $t i m e t=t-(r / c)$. The dynamic displacement is found from this function by the relation

$$
\begin{aligned}
z & =-\frac{\partial}{\partial r}\left(\frac{\psi(\tau)}{r}\right) \\
& =\frac{\psi}{r^{2}}+\frac{1}{r c} \frac{\partial \psi(\tau)}{\partial \tau}
\end{aligned}
$$

This equation divides the displacement into its induction field and its radiation field components, the first varying as $1 / r^{2}$ and the second as $1 / r$.

The reduced velocity potential (RVP) is the time derivative of the reduced displacement putential (RDP):

$$
\begin{aligned}
* & =\frac{\partial \psi(\tau)}{\partial \tau} \\
& =\frac{\partial \psi(\tau)}{\partial T}
\end{aligned}
$$

This relation leads to the corresponding relation for velocity:

$$
v=-\frac{\partial}{\partial r}\left(\frac{\phi(\tau)}{r}\right)
$$

It is seen that the form of RVP given by Eq. (A-4) is the same as that of the radiation field displacement in Eq. (A-2) so that a determination of the RVP is equivalent to finding the radiation field disflacemenc. This relation is given explicitly by the equation

$$
z_{r}=\phi / \mathrm{rc}
$$

On the other hand, the RDP contains both induction field and radiation field displacements, Residual RDP, sometimes called $\psi(\infty)$, is independent of 
retarded time $\tau$, so that $\partial \psi(\infty) / \partial \tau=0$; thus the only effect of $\psi(\omega)$

is seen in the induction field displacement. Consequently, residual RDP can be used as an adequate seismic coupling indicator only when the far-field displacement is a particular function of the near-field displacement from one case to another.

In the early work on decoupling, it was assumed that detection of underground events would be ac teleseismic distances. Because of differential earth attenuation, only frequencies below about $1 \mathrm{~Hz}$ were regarded as being important in the analysis of Latter et al..$^{2}$ This frequency is lower than the frequency $\omega_{0}$ defined by the relation $\omega_{0}=c / a$ foi a cavity or deformed region of reasonable size, whers a is the elastic radius. For a frequency of $1 \mathrm{hz}$ and a sonic velocity in the surrounding medium of $4,357 \mathrm{~m} / \mathrm{s}$, the radius a equals $693 \mathrm{~m}$. Consequently, in Latter's work, $w \ll w_{0}$.

The Fourier transform for the displacement has the form

$$
\hat{z}(f)=\frac{\hat{p a}}{4}\left(\frac{1}{r^{2}}+\frac{j \omega}{r c}\right) \frac{c^{2}}{\omega_{n}^{2}-j \omega_{0} \omega-\frac{(\lambda+2 \mu)}{4 \nu} \omega^{2}} .
$$

Sometimes this is expressed in cerms of $z(w)$, with the right-hand side divided by $2 \pi$. Even though w terms appear in this expression, the function $z(f)$ is defined here by the Fourier relation

$$
i(f)=\int_{n}^{\infty} z(t) \exp (-j 2 \pi f t) d t
$$

and the inverse cransform is

$$
z(t)=\int_{-\infty}^{\infty} \hat{z}(f) \exp (j 2 \pi f t) d f .
$$

For the radiation field contribution, only that portion on the regt-hand side of Eq. (A-7) multipled by jw/rc is retained. For $\omega \ll \omega_{0}$, this equation reduces to the form

$$
\hat{z}(t)=\frac{\hat{p}^{3}}{4 \mu c} \frac{j \omega}{r}
$$


When the pressure function is a step of amplitude $P_{0}$ at $t=0$, its transform is

$$
\hat{p}=\frac{p_{0}}{j \omega}
$$

The resulting spectral form for $z$ at low frequency is

$$
z(t)=\frac{p_{0} a^{3}}{4 \mu c} \frac{1}{r} .
$$

This result is also valid for other input pressure profiles that contain only high-frequency variations from the assumed step rise in pressure. This spectrum is independent of $\omega$ for values of $\omega \ll \omega_{0}$, i.e., the spectrum is flat at the low frequency end. When $\omega$ increases to the value $\omega=\omega_{0}$ (the corner frequency) this conclusion no longer applies.

In the elastic domain, the residual displacement is celated to the residual displacement potential by the relation

$$
\psi(\infty)=r^{2} z(\infty)
$$

From elasticity theory, the static displacement in this geometry is

$$
z=\frac{p a^{3}}{4 u r^{2}} .
$$

This relation is independent of the path followed in building up the pressure p. Consequently, the residual RDP is given by the relation

$$
\psi(\infty)=\frac{p a^{3}}{4 \nu} .
$$

where $a$ is the elastic radius and $p$ is the final radiai stress at that radius. Consequently, the radiation field displacement spectrum at zero frequency may be expressed, from Eqs. $(A-11)$ and $(A-14)$, 


$$
\hat{z}(f=0)=\frac{\psi(\infty)}{c r} .
$$

This is a form of a result obtained by Latter et al. ${ }^{2}$ It has alio been used by Murphy 27 in an analysis of sterling.

A simple mathematical derivation of Eq. (A-16) can be made by setting $F=0$ in $E q \cdot(A-8): \hat{z}(0)=\int_{0}^{\infty} z(\tau) d t$. For the radiation field $z(\tau)=(1 / \mathrm{rc}) \partial \psi / \partial \tau$, so that this substitution is made for $z(\tau)$ in the integral. Direct integration then gives the result $\hat{z}(f=0)=(1 / \mathrm{rc}) \psi(\infty)$.

In summary, the RDP gives the $y$-intercept of the displacement-frequency plot for the radiation field displacement. This line extends horizontally to higher frequencies until the natural frequency $\omega_{0}$ is approached. The residual RDP gives no information on this digplacement behavior at higher frequencies. 


\section{APPENDIX B: SOLUTION OF DYNAMIC EQUATIONS WITH FFT}

The Fast Fourier Transform method (FFT) is the conventional method of obtaining numerical Fourier series solutions with computers. This FFT specialization of the Fourier transform method does not affect the discussion of the solutions of linear differential equations here.

The Fourier transform of the equation relating displacement to driving pressure at some cavity radius or at some reference radius in the elastic medium is given by $E q$. $(A-7)$. It is convenient to make the substitutions listed in Table B-l to obtain the simpler kinematic relations among the dimensionless variables, as given in Table $B-2$.

In these equations the dimensionless pressure $\sigma$ can be considered as the driving function, the remainder of the right-hand side as the transfer function, and the left-hand side as the response function. In the equation for displacement, the term $1 / x+j n$ represents the sun of the induction and the radiation field components respectively, and for velocity the corresponding term is $\mathrm{j} n / \mathrm{x}-\mathrm{n}^{2}$.

Fourier series transform methods are routinely employed in electrical circuit theory, where the cransfer function is that of a filter relating oulput to input. In the FFT routine in the SOCKITTOME code at this laboratory, the filter is defined as one polynomial in jn divided by another in the same variable. Consequently the transfer function is simply specified by giving the proper coefficients from the dimensionless equations as the FFT input, and the dimensionless pressure history is given in numerical form.

In some cases this FFT procedure leads to nonconvergent results. Convergence can then be obtained by using as input the actual pressure time history over a period of time long enough to cover all transients of nonzero frequency, and then adding a similar pressure history of reversed sign. In spectral studies, points down to lower frequencies on the frequency spectrum can be obtained by extending the length of the input pressure history, even though the pressure remains constant at late times. 
TABLE B-1. Conversion to dimensionless units.

\begin{tabular}{|c|c|}
\hline Quantity & Conversion relation \\
\hline Radial distance & $x=-\frac{I}{a}$ \\
\hline Radial displacement & $\xi=\frac{z}{a}$ \\
\hline Time & $\tau=\frac{c t}{a}$ \\
\hline Velocity & $s=\frac{v}{c}$ \\
\hline Angular frequency & $\eta=\frac{\omega}{\omega_{0}}=\frac{a \omega}{c}$ \\
\hline & where $\omega_{0}=\frac{c}{a}$ \\
\hline Circular frequency & $\nu=\frac{\eta}{2 \pi}$ \\
\hline Lame constants & $\theta=\frac{\lambda}{4 \mu}+2 \mu$ \\
\hline Radial stress & $\sigma=\frac{p}{4 \mu}$ \\
\hline Reduced displacement potential & $\psi=\frac{\Psi}{a^{3}}$ \\
\hline Reduced velocity potential & $\phi=\frac{\phi}{a^{2} c}$ \\
\hline
\end{tabular}


TABLE B-2. Transformed kinematic relations.

Dimensionless variable

Equation

Reduced displacement potential

$$
\hat{v}=\frac{\hat{a}}{1+j \eta-B \eta^{2}}
$$

Reduced velocity potential

$\hat{t}=\frac{j_{n} \sigma}{1+j \eta-\beta \eta^{2}}$

Displacement

$\bar{\xi}=\frac{\hat{\sigma}}{x}\left[\frac{1}{x}+j \eta\right]\left[\frac{1}{1+j \eta-\beta \eta^{2}}\right]$

velocity

$\bar{\xi}=\frac{\hat{\sigma}}{x}\left[\frac{j_{n}}{x}-n^{2}\right]\left[\frac{1}{1+j n-\beta n^{2}}\right]$

$\tilde{\sigma}_{x}=\frac{\hat{\sigma}}{x} \frac{\frac{1}{x^{2}}+\frac{j}{x}-\beta n^{2}}{1+j n-\beta n^{2}}$

Additional relations

$\tilde{\zeta}=\mathrm{j} n \hat{\xi}$

$\dot{\varphi}=j n \hat{v}$ 
When the medium near the cavity responds inelastically to the overburden stress, the resulting stress field beromes more complex than for the elastic case. The form of the shear failure curve must be known before this more complicated final stress distribucion can be calculated. Latcer, Martinelli, Mathews, and McMillan ${ }^{3}$ have solved this problem for a medium that follows the shear stress relation

$$
\sigma_{r}-\sigma_{t}=\left\{\begin{array}{ll}
2 \mu \varepsilon & \left|\sigma_{r}-\sigma_{t}\right| \leq \sigma_{0} \\
2 \alpha \varepsilon+\sigma_{0}(1-\alpha / \mu) & \left|\sigma_{r}-\sigma_{t}\right| \geq \sigma_{0}
\end{array} .\right.
$$

The quantity $\sigma_{0}$ is twice the shear strength for the medium, $\mu$ is the elastic modulus of rigidity, and $\alpha$ is the slope of the failure curve beyond the initiation of failure. This stress-strain curve is shown in Fig. 22. The shear strain is given by the relation

$$
\varepsilon=\frac{\partial \xi}{\partial r}-\frac{\xi}{r}
$$

where $f$ is a radial displacement.

This shear stress relation is independent of confining pressure, and therefore the failure might be termed von Mises failure. The shear stress is related to shear strain through the modulus of rigidity in the usual way until a failure point is reached, $\sigma_{0} / 2$ in $\mathrm{Fig} . \mathrm{C}-1$. At this point there is not a sudden failure, but a continuation of the curve at the second slope $a$. Measurements by Heard et al. shown in Fig. 6 indicate that this gradual failure must occur in salt.

Latter et al. ${ }^{3}$ used the stress equilibrium equation

$$
\frac{\partial \sigma_{\tau}}{r}+\frac{2}{r}\left(\sigma_{\tau}-\sigma_{t}\right)=0
$$

and the compressibility equation

$$
\overline{3}\left(\sigma_{r}+2 \sigma_{t}\right)+P_{0}=k\left(\frac{\partial \xi}{\partial r}+\frac{\xi}{r}\right)
$$




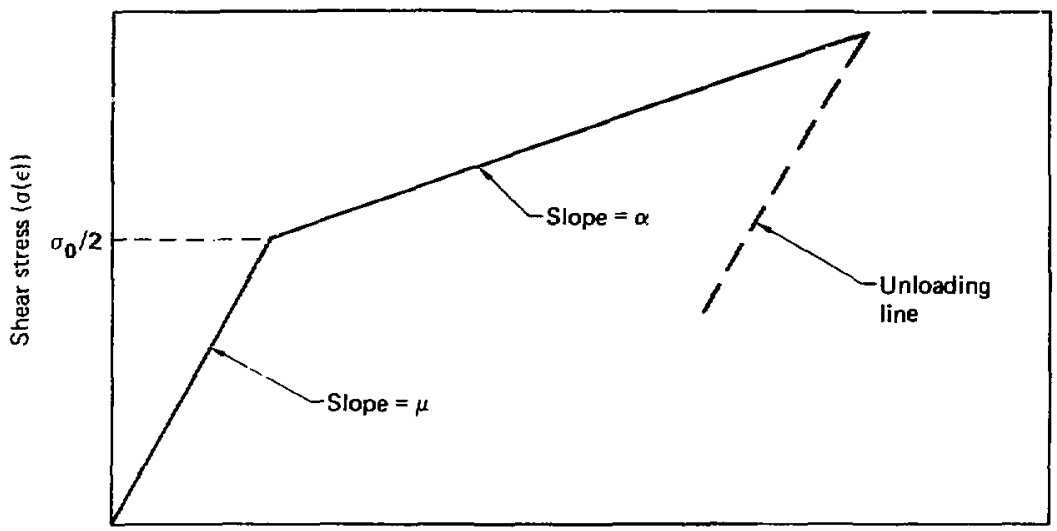

Shear strain ( $\epsilon)$

EIG. C-1. Stress-strain curve used by Latter ec al. ${ }^{3}$ in their anaiysis of stress relaxation around a cavity.

where $k$ is bulk modulus, and found the resulting stress distribution in the neigh'rorhood of the cavity. They used the boundary conditions that $\sigma_{r}=0$ at the cavity wall, that the radius stress $\sigma_{r}$ was continuous across the elastic-inelastic boundary, and that the stress state at infinity was a simple compression $\mathrm{P}_{0}$, and obtained the following relation for the elastic-plastic radius $\rho$ :

$$
\ln \frac{\rho}{a}+\frac{1}{3}-\frac{P_{O}}{4 \sigma_{F}}+\frac{k+4 u / 3}{k} \frac{a / \mu}{1-\frac{a}{\mu}}\left[\frac{1}{3}\left(\frac{\rho}{a}\right)^{3}-\frac{P_{o}}{4 \sigma_{F}}\right]-0
$$

The quantity $\sigma_{f}$ is the shear strength of the salt, equal to $\sigma_{0} / 2$ in Fig.c-1. (In this derivation the shear failure is assumed to depend only on the principal stresses $\sigma_{1}$ and $\sigma_{3}$. The concept of the octahedral shear strength is not used.)

The stresses and the displacement $\xi$ in the elastic regior ( $r>p$ ) are: 


$$
\begin{aligned}
\sigma_{s} & =\left(\frac{\rho}{r}\right)^{3} \sigma_{f} \\
\sigma_{r} & =-P_{o}+\frac{4}{3} \sigma_{s} \\
& =-P_{o}+\frac{\mu}{3}\left(\frac{\rho}{r}\right)^{3} \sigma_{E} \\
\sigma_{t} & =-P_{0}-\frac{2}{3}\left(\frac{\rho}{r}\right)^{j} \sigma_{E} \\
E & =-\frac{\sigma_{E}}{3}\left(\frac{\rho}{r}\right)^{2}
\end{aligned}
$$

In the inelastic region, $(r<0)$, the stresses and displacement are

$$
\begin{aligned}
& \sigma_{s}=\frac{k}{k+4 a / 3} \frac{a}{\mu} \sigma_{E}\left[\frac{k+4 \mu / 3}{k}\left(\frac{\rho}{r}\right)^{3}+\frac{\mu}{a}-1\right] \\
& \sigma_{r}=P_{o}+\frac{4 u_{s}}{3}+\frac{4 k}{k+4 a / j}(1-\alpha / \mu) \sigma_{E} \ln \frac{\rho}{r} \\
& \sigma_{t}=-\quad+\frac{2 \sigma_{s}}{3}+\frac{4 k}{k+4 a / 3}(1-\alpha / \mu) \sigma_{f} \ln \frac{\rho}{r} \\
& 5=-\left(\frac{k+4 u / 3}{k+4 x / 3}\right) \frac{\sigma_{f}^{\rho}}{3 \mu}\left(\frac{\rho}{r}\right)^{2}+\frac{4}{3}\left(\frac{\sigma_{f}(1-\alpha / \mu)}{k+4 a / 3}\right) r \ln \left(\frac{\rho}{r}+\frac{1}{3}\right)
\end{aligned}
$$

$\mathrm{J}^{+}$is noted that this is an infinitesimal strain solution, which cannot br. vaitd for a canjty created by an explosion. The boundarv conditions as used gave no incication as. J whether extra mass was present outside the cavity. Instead, the problem was solved as one where a cavity existed in a strtss-free medium, and the overburden pressure was then applied gradurlly. The displacements $\xi$ in Eqs. ( $C-6$ ) and $(C-7)$ are with respect to a medium under pressure $P_{a}$, iut with nc cavity present in che medium. The total displacement from the stress-free condition would be found by adding the term $-P_{0} r / 3 K$ to the right-raand sides or the disolacement equations, which represents the displacement with $e$ spect to the point $r=0$ caused by the unificm compression of the medium.

When the Poisson's ratio for the medium equals 0.25 , the buik compressibility is related $t j$ the shear modulus by the eqtation $k=5,3$. In addition, when the failure of the medium is of the von Mises type, as is 
the case for salt, then the value of a is zero. Under these conditions, the Latrer formulas can be simplified considerably.

The elastic-ineinstic interface is then given by the relation

$$
\ln \frac{D}{A}=\frac{P_{0}}{P_{f}}-\frac{1}{3} .
$$

The stresses and displacements in the elastic region $(r>\rho)$ are given by the relations

$$
\begin{aligned}
& \sigma_{s}=\left(\frac{\rho}{r}\right)^{3} \sigma_{f} \\
& \sigma_{T}=-P_{o}+\frac{4}{3}\left(\frac{\rho}{r}\right)^{3} a_{f} \\
& \sigma_{t}=-P_{0}-\frac{2}{3}\left(\frac{\rho}{r}\right)^{3} \sigma_{f} \\
& E=-\frac{a}{\sigma_{L}}\left(\frac{D}{r}\right)^{2} a_{f}
\end{aligned}
$$

The equations for the stresses and displacements in the inelastic region $(r<p)$ take the forms:

$$
\begin{aligned}
& \sigma_{s}=\sigma_{f} \\
& \sigma_{r}=-4 \sigma_{f} \ln \frac{r}{a} \\
& \sigma_{t}=-\left[\begin{array}{lll}
2 \ln \frac{r}{a}+1 & 2 \sigma_{f}
\end{array}\right] \\
& E=-\frac{3}{5} \frac{\rho}{\mu}\left(\frac{\rho}{r}\right)^{2} \sigma_{f}+\frac{4}{5} \frac{r}{L}\left[\ln \frac{\rho}{r}+\frac{1}{3}\right] \sigma_{f}
\end{aligned}
$$

Again the displacements are with respect to a medium compressed by overburden stress but with no cavity. 
The analytic displacement at the cavity wall is found by setting $r=a$ in Eq. (c-10) and using Eq. (C-8) to simplify the result. The final equation is

$$
\xi_{a}=\frac{a}{5 \mu}\left[-3\left(\frac{\rho}{a}\right)^{3} \sigma_{f}+P_{0}\right]
$$


Some information on decoupling can be gained from scaling arguments. If the quantity held constant is pressure nr energy per unit volume, then length and $t$ ime scale as the energy to the one-third power $\left(\mathrm{E}^{1 / \mathrm{s}}\right)$. If $1 \mathrm{kt}$ is the reference energy, and $a$ is the reference cavity radius, then a similarity solution wizl apply for $E \mathrm{kt}$ in a cavity of $\mathrm{E}^{1 / 3} \mathrm{a} \mathrm{m}$. Since RDP has the dimensions of $\mathrm{L}^{3}$, the RDP as measured at a distance $\mathrm{E}^{1 / 3}$ times the original dietance will be $E$ times the original RDP. The RDP is independent of distance, so RDP scales simply as $E$, for the case where the cavity is larger by the factor $E^{1 / 3}$.

The scaling for RVP differs from that for RDP. Since the dimensions of RVP are $L^{3} / T$, and since $c$ ine $T$ scales as $E^{1 / 3}$, the RVP varies as $E^{2 / 3}$ provided that the cavity radius is increased by the factor $E^{1 / 3}$, and provided all the medium remains in the elastic rang.-

For tamped explosions, the deformation should depend upon energy per unit volume, so that the radius of damage varies as $E^{l / 3}$. The scaling relations thus hold for inelastic motion around the source, so that the RDP varies as $E$, and the RVP varies as $E^{2 / 3}$.

Scaling displacements and velocities are more complicaced because these quantities depend on the radial distance. Direct scaling eives the result that displacement scales as $E^{1 / 3}$, but the point of observat on is also moved out by this factor of $E^{1 / 3}$. Since the inductive displacement varies as $1 / r^{2}$, moving back to the original observation point introduces an additional factor of $E^{2 / 3}$, giving the final result that the displacement associ.ted with the inductive field at a fixed observation point varies directly as $E$.

For the transient displacement associated with the radiation field, direct scaling gives the same $E^{1 / 3}$ variation of displacement at similar scaled distances. This radiative component of displacement varies as $1 / r$, so that the transient displacement associated with the radiation field varies as $E^{2 / 3}$. In seismic work the transient displacement in the radiation field is the one of primary interest.

Both induction and radiation field velocities remain unchanged in scaling $E$, but the distance to the observation point scales as $E^{1 / 3}$. Consequently the induction field velocity, which varies as $1 / r^{2}$, varies as $E^{2 / 3}$ at a fixed observation point. Similarly, the radiation field velocity varies as $\mathrm{E}^{1 / 3}$. 
The frequency spectrum of the RDP is defined by the relation

$$
\hat{v}(f)=\int_{t_{1}}^{t} \psi(t) \exp (j 2 \pi f t) d t .
$$

Although the dimensions of $\psi(t)$ are $L^{3}$, the dimensions of $\psi(f)$ are seen to be $\mathrm{L}^{3} \mathrm{~T}$. The function $\downarrow$ is independent of the position of the measuring point, assuming it 1 ies in the elastic region, go that direct scaling can be used. Consequencly, the amplitude of the $\tilde{u}(\mathrm{f})$ spectrum varies $a s \mathrm{E}^{4 / 3}$, while the frequency varies as $\mathrm{E}^{-1 / 3}$. In the same way it is found that the amplitude of the RVP spectrum (f) varies as E.

The amplitudes $a_{i}$ the inductive and radiative field displacements and velocities depend upon the radial distances, so that it is necessary to convert bacis to the original point of measurement to find the dependence on the input energy only. Denoting induction field and radiation field components by the subscripts $i$ and $r$ respectively, the results are $z_{i}\left(f_{r}\right.$ ) varies as $E^{4 / 3}, z_{\tau}(E)$ varies as $E, v_{i}(f)$ varies as $E$, and $v_{r}(f)$ varies as $\mathrm{F}^{2 / 3}$. The reference frequency $f$ varies as $\mathrm{E}^{-1 / 3}$. These results are listed in Table D- 1 .

TABLE D-i. Variation with explosion energy $E$ of seismic quantities at a fixed observacion point for the case where the elastic radius values as $E^{1 / 3}$.

\begin{tabular}{|c|c|c|}
\hline Quantity & Induction field & Radiation field \\
\hline RDP $-\psi \psi(t)$ & E & E \\
\hline RVP $--\varphi(t)$ & $E^{2 / 3}$ & $E^{2 / 3}$ \\
\hline Displacement $z(t)$ & E & $E^{2 / 3}$ \\
\hline velocity $v(t)$ & $E^{2 / 3}$ & $E^{1 / 3}$ \\
\hline$|\bar{\psi}(E)|$ & $E^{4 / 3}$ & $E^{4 / 3}$ \\
\hline$|\hat{\phi}(f)|$ & E & $\mathbf{E}$ \\
\hline$|\hat{z}(f)|$ & $E^{4 / 3}$ & $E$ \\
\hline$|\hat{v}(E)|$ & $\mathrm{E}$ & $E^{2 / 3}$ \\
\hline Time & $E^{1 / 3}$ & $E^{1 / 3}$ \\
\hline Frequency & $E^{-1 / 3}$ & $E^{-1 / 3}$ \\
\hline
\end{tabular}


Latter et al. ${ }^{2}$ have shown that the residual RDP is independent of cavity radius if the medium remains in the elastic range. The time scale is changed with a change in cavity radius, so that the effect of increasing the cavity radius is to shift the $z$ (f) vs frequency plot on a logarithmic scale to the left. The radiation fipld displacement spectrum resulting from the application of a step in pressure at an elastic radius a has been analyzed by Larson ${ }^{28}$ and by others, and has the form shown in Fig. D-1. Figure D-2 shows the corresponding radiacion field displacement vs time curve. If the cavity radius is increased by a factor of two but the total energy is kept constant, then the spectrum is shifted to the left by a factor of two on the logarithmic scale. The vertical height of the rlat portion of the spectrun and the amplitude of the zero-frequency component remain unchanged.

In the time domain, the increase in cavity size results in a broadening and lowering of the displacement-time curve. Therefore if peak radiation field displacement is the measure of coupling, then increasing the cavity size decreases the coupling. The peaking of the displacement curve for the smaller cavity is caused by the higher-frequency displacement contributions added vectorially to the lower frequency components as seen in Fig. D-2 for the larger cavity. Although the flat sections of the spectral amplitudes essentially coincide in Fig. D-2, the phases of these low frequency ccaponents

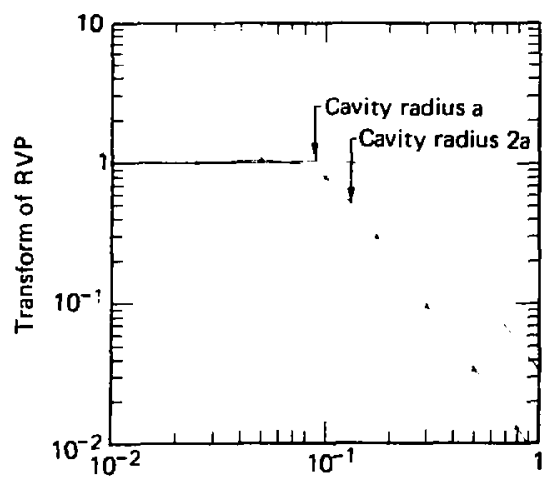

Dimensionless frequency

FIG. D-1. Effect of cavity radius on the RVP spectrum, with step pressure at tha cavity wall.

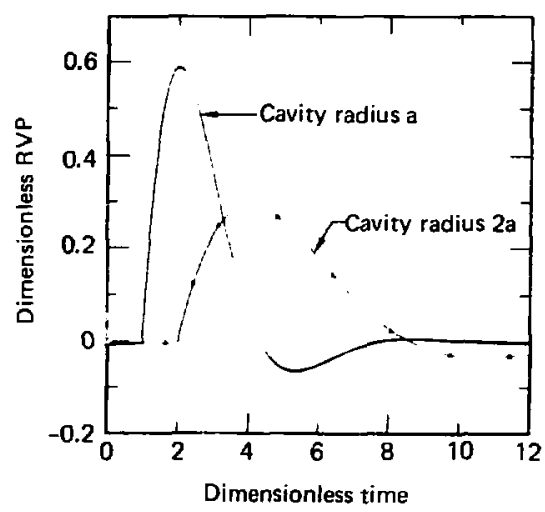

FIG. D-2. Effect. of the cavity radius on the RVP curve, with step pressure at the cavity wall. 
will differ. Ic is noted that the area under the radiation field displacement vs $t$ ime curve can be regarded as the amplitude of the zero frequency component, and that the areas under the curves for the two cavities are equal. If the energy $E$ is varied but the cavity radius remains constant, the medium remaining in the elastic domain, scaling arguments cannot be applied directly. Sharpe's solution, as presented in the next section, shows that the induction-field displacement varies as $a^{3} p$, where $p$ is cavity pressure, while radiation field displacement varies as $a^{2} p$. The time scale of the disturbance depends only on the cavity radius, and is independent of energy or pressure. Since the radius is held constant, however, the displacement varies only with pressure, which varies directly with energy.

In a similar manner, the velocity, which can be found as the time derivative of the displacement, also varies directly sith energy. The RDP and RVP as well as the amplitudes of the frequency spectra of these quantities, also vary directly as E. 


\section{APPENDIX E: RVP FROM THE SHARPE SOLUTION}

The Sharpe solution for the displacement resulting from a step in pressure applied to the inner surface of a spherical cavity in a medium where the Lamé constants $\lambda$ and $\mu$ are equal is the following:

$$
\begin{aligned}
z=\frac{a p}{4}\left\{( \frac { a } { r } ) ^ { 2 } \left[1-\sqrt{\frac{3}{2}}\right.\right. & \exp \left(-\frac{2}{3} \omega_{0} \tau\right) \sin \left(\frac{2 \sqrt{2}}{3} \omega_{0} \tau+\tan ^{-1} \sqrt{2}\right) \\
& \left.\left.+\sqrt{2}\left(\frac{a}{r}\right) \exp \left(-\frac{2}{3} \omega_{0} \tau\right) \sin \left(\frac{2 \sqrt{2}}{3}\right) \omega_{0} \tau\right]\right\} .
\end{aligned}
$$

Only the radiation field solution, which varies as $1 / r$, is of interest. It has the form

$$
z_{r}=\frac{\sqrt{2} a^{2} P}{4 \mu r} \exp \left(-\frac{2}{3} \omega_{0} \tau\right) \sin \left(\frac{2 \sqrt{2}}{3} \omega_{0} \tau\right) .
$$

The time at which maximum radiation field displacement occurs is found by differentiating chis expression with respect to $T$ and setting, the result equal to zero. This gives the relation

$$
\tan \left(\frac{2 \sqrt{2}}{3} \omega_{0} \tau_{\mathrm{m}}\right)=\sqrt{2}
$$

This value of $T_{m}$ is used in $E q .(E-2)$, with the following result:

$$
z_{m}=\frac{(.5876) a^{2} p}{4 \mu r}
$$

The term $p$ is found from Eq. (14), giving the marimum displacement $z_{m}$ as a function of input energy $E$ :

$$
z_{m}=\frac{3(.5876)(\gamma-1) E}{16 \pi a \mu r} .
$$

From Eq. $(A-2)$, it is seen that the peak RVP is related to the peak far-field displacement by the relation 


$$
\Phi_{\mathrm{m}} \times \mathrm{rez}_{\mathrm{m}}
$$

The peak RVP is therefore

$$
\phi_{m}=\frac{3(.5876)(\gamma-1) c E}{16 \pi a \mu} .
$$

The values $\gamma=1.2, c=4357 \mathrm{~m} / \mathrm{s}, E=1 \mathrm{kt}$, and $\mu=13.91 \mathrm{GPa}$ wera used from the example in the section, "Dynamic Calculations with the SoC Code--Step Input Pressures," above, in obtaining the numerical relation

$$
\Phi_{m}=\frac{9182}{a} \mathrm{in} \mathrm{m}^{3} / \mathrm{sec} .
$$


The wave equation of motion for the gas inside a spherical cavity is

$$
\frac{\partial^{2} \psi}{\partial t^{2}}-c^{2} \frac{\partial^{2} \psi}{\partial x^{2}}=0 \text {. }
$$

The general solution has the form

$$
\psi=\psi_{1}(r-c t)+\psi_{2}(r+c t) .
$$

A standing wave solution is obtained by setting $\psi_{1}=A \sin k(r-c t)$ and $\psi_{2}=A \sin k(r+c t)$, where $k$ is a constant. These terms can be combined to give the result

$$
\psi=2 A(\sin k r)(\cos k c t) .
$$

The solution for displacement obtained from this equation is

$$
\begin{aligned}
z & =-\frac{\partial(\psi / r)}{\partial r} \\
& =\frac{2 A}{r^{2}}(\sin k r)(\cos k c t)-\frac{2 A k}{r}(\cos k r)(\cos k c t)
\end{aligned}
$$

At $r=a, z=0$. This leads to the relation

$$
\begin{aligned}
& \sin k a-k a(\cos k a)=0 \\
& k a=\tan k a
\end{aligned}
$$

The firgt solution $k a=0$ gives the static result. The next solution is ka $=4.4935$, as det:rmined rumerically. The frequency associated with this second zero is found from $\cos 2 \pi \mathrm{ft}=$ $\cos k c t$, or

$$
\mathrm{f}=\frac{\mathrm{kc}}{2 \pi}=\frac{4.4935}{2 \pi} \frac{\mathrm{c}}{\mathrm{a}} \text {. }
$$


The period is

$$
\Delta t=\frac{2 \pi a}{4.4935 c}
$$

The corresponding period of resonance between two parallel plates separated by a distance $a$ is $2 a / c$. These results show that the spherical convergence raises the lowes $\llcorner$ mode resonalı frequency by the factor $4.4935 / \pi=1.43$.

Since it was assumed in this derivation that the velocity remained constant with respect to time and radial distance, this derivation is the acoustic approximation derivation. Actually, for large amplitudes of motion the sonic velocity at sơne radial distance changes with time, and the velocity at some point in time changes with radial distance. 